\title{
Improved Method for Airborne Photogrammetry Using Small Uninhabited Aerial Vehicles in Recording Existing and Historic Structures
}

\author{
by \\ Joshua Dalphy \\ A thesis submitted to \\ the Faculty of Graduate and Postdoctoral Affairs \\ in partial fulfilment of \\ the requirements for the degree of \\ Master of Applied Science \\ in \\ Aerospace Engineering \\ Ottawa-Carleton Institute for Mechanical and Aerospace Engineering \\ Department of Mechanical and Aerospace Engineering \\ Carleton University \\ Ottawa, Ontario, Canada
}

August 2017

Copyright (C)

2017 - Joshua Dalphy 
The undersigned recommend to

the Faculty of Graduate and Postdoctural Affairs

acceptance of the thesis

\title{
Improved Method for Airborne Photogrammetry Using Small Uninhabited Aerial Vehicles in Recording Existing and Historic Structures
}

\author{
Submitted by Joshua Dalphy \\ in partial fulfilment of the requirements for the degree of \\ Master of Applied Science
}

Dr. Jeremy Laliberté, Supervisor

Dr. Mario Santana, Co-Supervisor

Dr. Ron Miller, Department Chair

Carleton University

2017 


\section{Abstract}

The objective of this research was to investigate an alternative cost-effective means of improving current methods for airborne photogrammetry using small uninhabited aerial vehicles. Through added computational functionality, a custom data acquisition script was developed using Dronekit-Python to directly associate the aircraft's position and orientation to an image being captured in real time. The implemented system was tested by conducting an aerial survey on River Field, located on the campus of Carleton University. The captured images were used to generate three 3D point clouds, using the indirect, direct and assisted methods of georeferencing. A comparison between the data recorded from the aircraft's telemetry log with the results obtained from the custom data acquisition script was conducted to verify its efficacy. The calculated parameters of exterior orientation for the direct and assisted were then compared to the reference values determined through indirect georeferencing. Additionally, a comparison of the generated 3D point clouds was undertaken. The results showed good agreement between the point clouds generated through direct and assisted georeferencing with the reference, generated using the indirect method. 


\section{Acknowledgments}

I would first like to express my gratitude towards my supervisor Dr. Jeremy Laliberté for providing me with the opportunity to conduct interesting research in my field of interest. His advice and guidance have been of great help and it was a pleasure being part of your research group and working with you over the past two years. I would also like to thank my co-supervisor, Dr. Mario Santana for his help and expertise.

I am grateful to Dr. Fabio Remondino and all the members of the 3D Optical Metrology research group of the Fondazione Bruno Kessler, who hosted me during my internship. It was a great experience and talking with each one of you about topics relating to surveying and photogrammetry was a great help towards my research.

I would like to express my gratitude to my colleagues Prem Anand, Niall Mccallum, Salman Shafi and Loughlin Tuck for their time and effort in aiding me with the pre-flight work and during flight testing. I would like to express my thanks to the guys from the Minto 3041 office for creating a pleasant environment which was conducive to enlightening exchanges.

Lastly, I would be remiss if I did not thank my family for their continued support and encouragement. 


\section{Table of Contents}

Abstract $\quad$ iii

Acknowledgments $\quad$ iv

Table of Contents $\quad$ v

List of Tables viii

List of Figures $\quad$ ix

List of Acronyms xii

List of Symbols $\quad$ XV

1 Introduction 1

1.1 Uninhabited Aerial Vehicles for Heritage Documentation . . . . . . . 1

1.2 Motivation and Scope of Research . . . . . . . . . . . . . 5

1.3 Thesis Outline...................... 6

2 Literature Review $\quad 7$

2.1 Mathematical Background . . . . . . . . . . . . . . 7

2.1.1 Description of Position, Orientation and Frame . . . . . . 7

2.2 UAVs for Aerial Surveying . . . . . . . . . . . . . . 10

2.3 Principles of Georeferencing . . . . . . . . . . . . . . . . . 16 
2.3.1 The Collinearity Equation . . . . . . . . . . . . . . 16

2.3 .2 Indirect Method . . . . . . . . . . . . . . . . . . . . . . 21

2.3 .3 Direct Method . . . . . . . . . . . . . . . . . . . 24

2.3.4 Integrated Sensor Orientation (ISO) . . . . . . . . . . . 31

2.3.5 Relevant Work . . . . . . . . . . . . . . . . . . . . . . 32

3 Experimental Methodology 35

3.1 System Overview . . . . . . . . . . . . . . . . . . . . . 35

3.1 .1 Aircraft Hardware . . . . . . . . . . . . . . . . 36

3.1.1.1 3D Robotics Iris+ Uninhabited Aerial Vehicle . . . . 37

3.1.1.2 Pixhawk PX4 Autopilot . . . . . . . . . . . . . 38

3.1.1.3 Raspberry Pi 3B Single Board Computer . . . . . . . 39

3.1.1.4 Raspberry Pi Camera V2 . . . . . . . . . . . . 40

$3.1 .2 \quad$ Software . . . . . . . . . . . . . . . . . 42

3.1.2.1 Mission Planner . . . . . . . . . . . . . . . . 42

3.1.2.2 Dronekit. . . . . . . . . . . . . . . 45

3.1.2.3 Agisoft PhotoScan . . . . . . . . . . . . 46

3.2 Testing . . . . . . . . . . . . . . . . . . . . . . 48

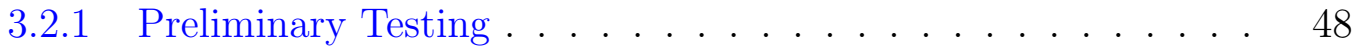

$3.2 .2 \quad$ River Field Case Study . . . . . . . . . . . . . . . . . . . 52

3.2.2.1 Special Flight Operations Certificate . . . . . . . 53

3.2 .2 .2 Preflight work . . . . . . . . . . . . . 54

$3.2 .2 .3 \quad$ Flight $1 \ldots \ldots \ldots \ldots$

3.2 .2 .4 Flight $2 \ldots \ldots \ldots \ldots \ldots \ldots \ldots$

4 Results and Discussion $\quad 64$

4.1 Data Acquisition Script . . . . . . . . . . . . . . . . . . 65

4.2 Georeferencing Results . . . . . . . . . . . . . . 75 
5 Conclusions and Future Work $\quad 98$

5.1 Conclusions . . . . . . . . . . . . . . . . . . . . . . . . . 98

5.2 Future Work . . . . . . . . . . . . . . . . . . 100

$\begin{array}{ll}\text { References } & 102\end{array}$

$\begin{array}{lll}\text { Appendix A CloudCompare Results } & 107\end{array}$

A.1 Indirect versus Direct . . . . . . . . . . . . . . . . . 108

A.2 Indirect versus Assisted . . . . . . . . . . . . . . . . . . . . 110 


\section{List of Tables}

2.1 Sub-categories of tactical UAVs . . . . . . . . . . . . . . 11

2.2 Summary of the various applications of UAVs in the field of geomatics 12

3.1 Pixhawk mechanical properties . . . . . . . . . . . . . . 39

3.2 Raspberry Pi Camera V2 physical characteristics . . . . . . . . . . . 42

3.3 Emlid Reach RTK GPS mechanical properties . . . . . . . . . . 56

3.4 Ground control point measurements . . . . . . . . . . . . . . 59

4.1 Average position of the ground control points . . . . . . . . . . 80

4.2 Position coordinates relative to the UAV's IMU . . . . . . . . . . 84

4.3 Absolute averaged difference between indirect and direct results . . . 86

4.4 Absolute average difference between indirect and assisted results . . . 88

4.5 Georeferencing processing times . . . . . . . . . . . . . . . . 91 


\section{List of Figures}

1.1 Available techniques for 3D recording . . . . . . . . . . . . 3

1.2 UAV workflow for image acquisition . . . . . . . . . . . . 4

2.1 Vector shown relative to frame . . . . . . . . . . . 8

2.2 Position and orientation . . . . . . . . . . . . . . . 9

2.3 Ground sampling distance . . . . . . . . . . . . . . . . . . . 14

2.4 a) manual flight; b) low cost navigation system assisted flight; c) high precision automated flight and image acquisition . . . . . . . . 15

2.5 Relation between image and object plane . . . . . . . . . . . 16

2.6 Collinearity model of point P projected on to the image plane . . . . 18

2.7 Targeted ground control point. . . . . . . . . . . . . . 22

2.8 Relation between image and object plane . . . . . . . . . . . . 23

2.9 Flow chart comparing traditional and direct georeferenced photogram-

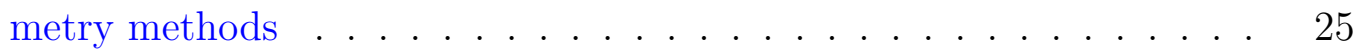

2.10 Illustration of the general notion of direct georeferencing . . . . . . . 27

2.11 Visual representation of the misalignment between the camera and body frames . . . . . . . . . . . . . . . . . . 28

3.1 System overview . . . . . . . . . . . . . . . . . 36

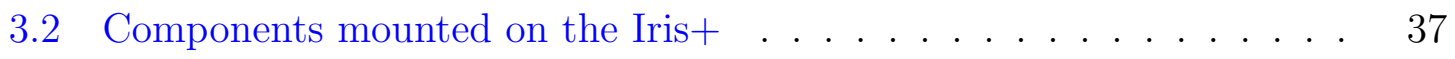

3.3 Pixhawk PX4 . . . . . . . . . . . . . . . . . . . . 39

3.4 Raspberry Pi model 3B . . . . . . . . . . . . . . . . . . . 40 
3.5 Raspberry Pi camera V2 . . . . . . . . . . . . . . . . 41

3.6 Mission Planner GUI . . . . . . . . . . . . . . . . . . . . . . . . . 43

3.7 Mission Planner MAVLink mission command list . . . . . . . . . . . 44

3.8 Connection between Raspberry Pi and Pixhawk autopilot . . . . . . . 49

3.9 Data acquisition script flowchart . . . . . . . . . . . . 50

3.10 Ground control station set-up at the Carleton River Field test site . . 52

3.11 River Field Google satellite image . . . . . . . . . . . . . . . . 53

3.12 Ground control point . . . . . . . . . . . . . . 55

3.13 Emlid Reach RTK GPS module and antenna . . . . . . . . . . . . . 57

3.14 RTK GPS mounted on tripod . . . . . . . . . . . . 58

3.15 First test flight navigation plan . . . . . . . . . . . . . . 60

3.16 Second test flight navigation plan . . . . . . . . . . . . . 62

3.17 System overview . . . . . . . . . . . . . . . 63

4.1 Comparison between the UAV's position logged by the Pixhawk and the custom Python data acquisition script . . . . . . . . . . . 66

4.2 Comparison between the UAV's altitude logged by the Pixhawk and the custom Python data acquisition script . . . . . . . . . . . 67

4.3 Comparison between the UAV's altitude logged by the Pixhawk and the custom Python data acquisition script corrected . . . . . . . . . . 68

4.4 Comparison between the UAV's roll logged by the Pixhawk and the custom Python data acquisition script showing turn numbers . . . . . 70

4.5 Comparison between the UAV's pitch logged by the Pixhawk and the custom Python data acquisition script showing turn numbers . . . . . 72

4.6 Comparison between the UAV's yaw logged by the Pixhawk and the custom Python data acquisition script . . . . . . . . . . . . 74

4.7 Initial generated 3D point cloud using data from flight test $1 \ldots$. . . 77

4.8 Combined flight paths. . . . . . . . . . . . . . 78 
4.9 3D point cloud generated using the indirect method of georeferencing. 79

4.10 Visual representation of different height measurement conventions . . 80

4.11 Direct georeferencing vectors. . . . . . . . . . . . . . 82

$4.123 \mathrm{D}$ point cloud generated using the direct method of georeferencing. . 85

4.13 3D point cloud generated using the assisted method of georeferencing. $\quad 87$

4.14 Indirect and direct 3D point clouds shown in CloudCompare. . . . . . 93

4.15 Indirect and direct 3D cloud-cloud distance comparison . . . . . . . . 94

4.16 Distribution of the cloud to cloud absolute distance between the indirect and direct point clouds for the $\mathrm{x}$ axis . . . . . . . . . . . . . 95

4.17 Indirect and assisted 3D cloud-cloud distance comparison . . . . . . . 96

A.1 Distribution of the cloud to cloud absolute distance between the indirect and direct point clouds for the y axis . . . . . . . . . . . 108

A.2 Distribution of the cloud to cloud absolute distance between the indirect and direct point clouds for the $\mathrm{z}$ axis . . . . . . . . . . . . . 109

A.3 Distribution of the cloud to cloud absolute distance between the indirect and assisted point clouds for the $\mathrm{x}$ axis . . . . . . . . . . . . . . 110

A.4 Distribution of the cloud to cloud absolute distance between the indirect and assisted point clouds for the y axis . . . . . . . . . . . . . 111

A.5 Distribution of the cloud to cloud absolute distance between the indirect and assisted point clouds for the $\mathrm{z}$ axis . . . . . . . . . . . . 112 


\section{List of Acronyms}

\begin{tabular}{ll} 
Acronyms & Definition \\
\hline \hline 3DR & 3D Robotics \\
AGL & Above ground level \\
API & Application program interface \\
ASL & Above sea level \\
AUVSI & The Association for Unmanned Vehicle Systems International \\
CEP & Circular error probability \\
CSI & Camera serial interface \\
DGPS & Differential Global Positioning System \\
DSI & Display serial interface \\
DTM & Digital terrain model \\
EGNOS & European Geostationary Navigation Overlay Service \\
EKF & Extended Kalman filter \\
FPU & Floating point unit \\
\hline
\end{tabular}




$\begin{array}{ll}\text { FPV } & \text { First person view } \\ \text { GPIO } & \text { General purpose input/output } \\ \text { GPS } & \text { Global Positioning System } \\ \text { GSD } & \text { Ground sampling distance } \\ \text { GUI } & \text { Graphical user interface } \\ \text { HDMI } & \text { High-definition multimedia interface } \\ \text { IMU } & \text { Inertial measurement unit } \\ \text { INS } & \text { Inertial navigation system } \\ \text { ISO } & \text { Integrated sensor orientation } \\ \text { LIDAR } & \text { Light detection and ranging } \\ \text { MAV } & \text { Micro aerial vehicles } \\ \text { RSAS } & \text { Multi-Functional Satellite Augmentation System } \\ \text { RP } & \text { Recincipal point } \\ \text { RAM } & \text { Random access memory } \\ \text { RPE } & \text { Radio frequency } \\ \text { Remotely piloted aerial system }\end{array}$




$\begin{array}{ll}\text { SBAS } & \text { Satellite-Based Augmentation System } \\ \text { SFOC } & \text { Special Flight Operation Certificate } \\ \text { SSH } & \text { Software-in-the-loop } \\ \text { TELEM } & \text { Tecure shell } \\ \text { Tx } & \text { Transmission } \\ \text { UAV } & \text { Uninhabited } \\ \text { USB } & \text { Universal serial bus } \\ \text { UTM } & \text { Universal Transverse Mercator } \\ \text { UVS } & \text { Unmanned vehicle systems } \\ \text { VFR } & \text { Visual flight rules } \\ \text { VTOL } & \text { Vertical takeoff and landing } \\ \text { WAAS } & \text { Wide Area Augmentation System } \\ \text { WGS84 } & \text { World Geodetic System 1984 }\end{array}$




\section{List of Symbols}

\begin{tabular}{ll} 
Symbols & Definition \\
\hline \hline$\alpha$ & Roll angle \\
$\beta$ & Distance between flight lines \\
$B$ & Pitch angle \\
$\gamma$ & Baselength \\
$c$ & Yaw angle \\
$d$ & Camera's focal length \\
$F_{b}$ & Pixel size \\
$H$ & Mround area of one photograph \\
$H_{A S L}$ & Orthonometric height \\
$h$ & Ellipsoidal height \\
$\kappa$ & Angle of rotation about the Z axis \\
$l$ & Percentage of forward overlap
\end{tabular}




$\begin{array}{ll}m_{b} & \text { Photo scale number } \\ O & \text { Geoid height } \\ P & \text { Position of camera station } \\ q & \text { Point located in object space } \\ \mathbf{R} & \text { Percentage of side overlap } \\ r_{i j} & \text { Element of the rotation matrix } \\ s & \text { Scale } \\ \theta & \text { Offset angle between camera and body reference frame } \\ \omega & \text { Angle of rotation about the X axis } \\ & \end{array}$




\section{Chapter 1}

\section{Introduction}

\subsection{Uninhabited Aerial Vehicles for Heritage Doc- umentation}

The continuing research and development of new digital technologies has had a significant effect on the documentation and recording of historical and archaeological sites, structures and landscapes. The documentation and preservation of historically significant sites enables a connection to be made between the past and present as well as to develop an appreciation and understanding on how previous generations of people lived. Moreover, it provides an opportunity to study the materials and techniques which were used during their construction. However, these sites are often at risk of being damaged or in the worst case destroyed by environmental calamities, vandalism, war and erosion over time [1]. Such events reinforce the need for proper documentation of historical sites as they can provide guidance when the need arises for repairs and retrofitting of existing structures. If the sites are damaged beyond repair, the images and notes taken prior to the loss can be used to educate future generations on previous/ancient construction techniques [1]. Thus, it is important to choose an appropriate method for documentation based on the requirements and cost 
of the project. One such method is aerial photogrammetry using UAVs.

In recent years, the use of uninhabited aerial vehicles (UAVs) has become more common in many civil applications, with an estimated total economic impact of 82 billion dollars US to be generated between 2015 and 2025 [2]. The Association for Unmanned Vehicle Systems International (AUVSI) expect that by 2025, over 100000 new jobs will be created in the United States as a result of UAVs being adopted by society for various applications [2]. Their use in the field of architectural and archaeological surveying for heritage conservation has gained popularity due to their low cost and ability to conduct rapid surveys relative to traditional methods of documentation [3]. Moreover, UAVs can be employed to map and survey areas that are otherwise inaccessible or have been deemed too dangerous for manned aircraft or terrestrial surveying techniques. Primarily, UAVs are used for image acquisition in order to generate 3D point clouds, digital terrain models (DTM) and ortho-images which can produce results with high accuracy and spatial resolution [3]. Figure 1.1 illustrates the various available methods, techniques and platforms for 3D recording in terms of both size and complexity. The figure shows that among the available techniques, UAVs are ideal for small (approximately $1 \mathrm{~m}$ ) to medium size (approximately $10 \mathrm{~km}$ ) scenes of varying complexities (ranging from 1000 points to over 10 million). 


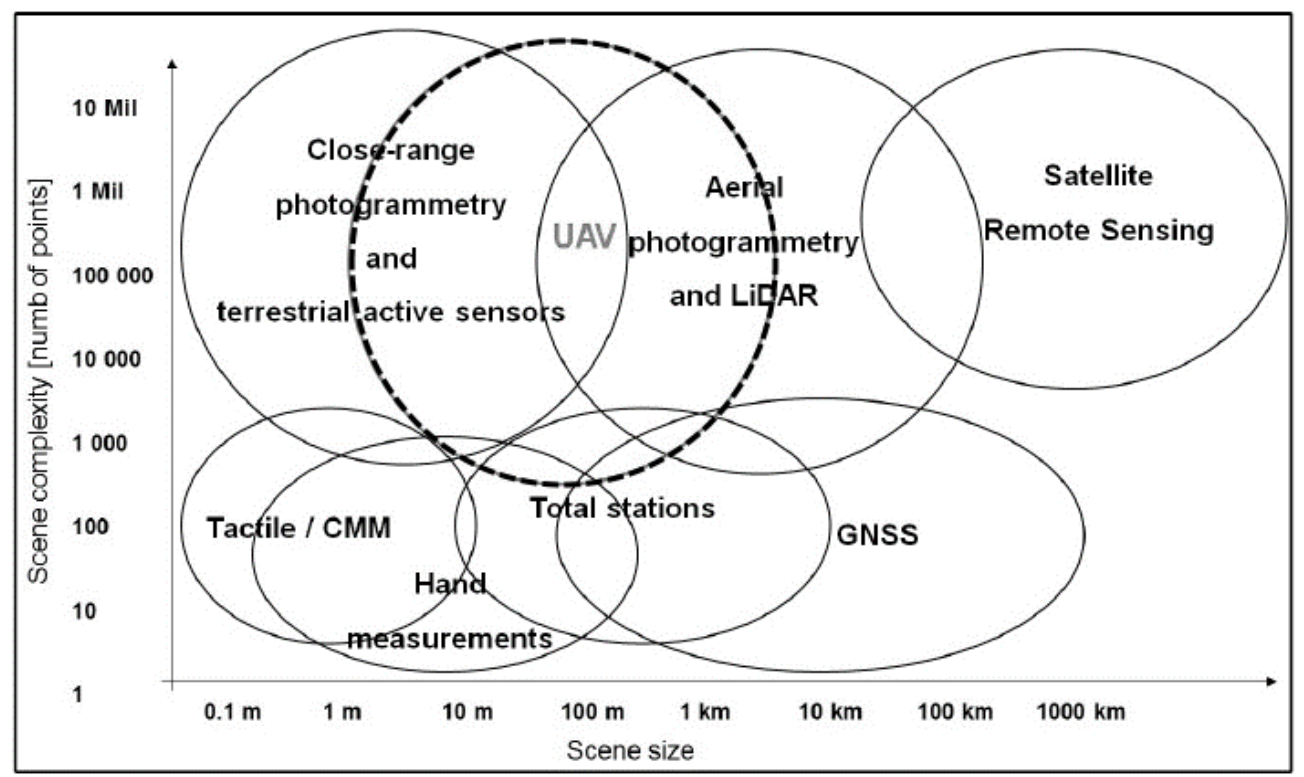

Figure 1.1: Available techniques for 3D recording [1]

The typical workflow for aerial surveying is shown in Figure 1.2. This figure provides a visual representation of each of the steps involved in the process, from mission planning to DTM generation and three-dimensional modeling. Moreover, Figure 1.2 also identifies various parameters that require consideration at each point in the workflow. 


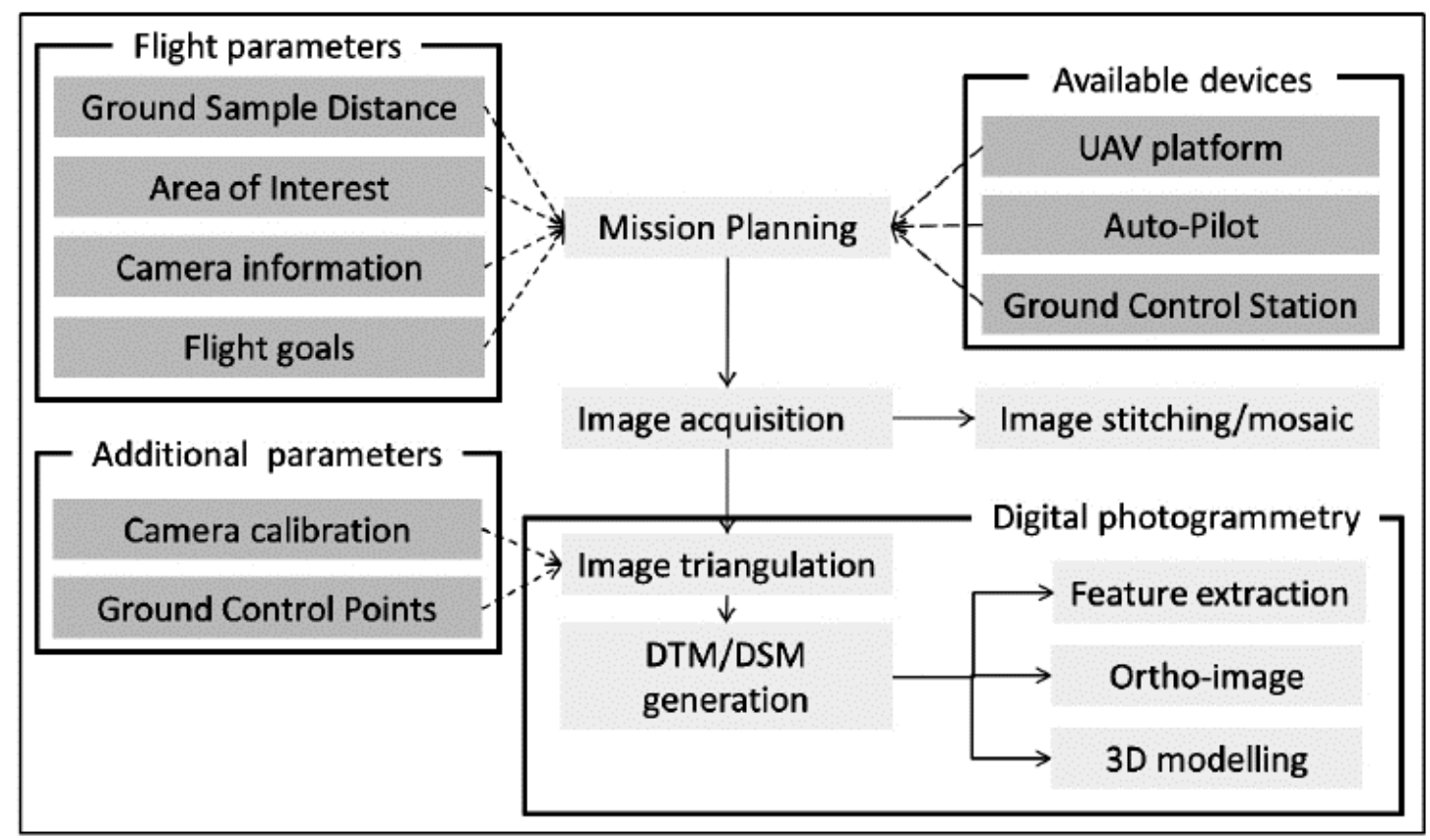

Figure 1.2: UAV workflow for image acquisition [1]

Images are captured using one of four different methods; underwater, terrestrial, aerial or satellite imaging. However, this thesis will only focus on the method of aerial image acquisition. Specifically, this thesis will look at the use of multi-rotor UAVs for capturing digital images. The processing of captured digital images can be conducted in one of three ways; indirect georeferencing, direct georeferencing and integrated sensor orientation (ISO), which combines the features of both the direct and indirect methods [4]. Traditionally, the indirect method has been the primary approach employed in the field. However, with the continuing advancement and development of new GPS and inertial measurement unit (IMU) technologies in recent years, direct georeferencing has started to gain favour. Direct georeferencing can directly determine the parameters of exterior orientation and is gaining popularity as it can decrease the overall cost of the aerial mapping and surveying process by reducing the total number of ground control points being used and can alleviate some of the post-processing of the results [5]. Direct georeferencing is primarily limited by 
four factors: the image modeling sensor, sensor placement, sensor synchronization and sensor errors, each of which will be discussed in detail in Chapter 2. However, with new UAV systems each having their own unique sensors and equipment being released every year and the ability to customize already existing models, this provides an opportunity to further research the topic of direct georeferencing and data acquisition using onboard sensors in an attempt to improve the performance of currently available methods.

\subsection{Motivation and Scope of Research}

The aim of this thesis research is to investigate an alternative cost effective means of direct georeferencing and data acquisition for a multi-rotor UAV system using the available onboard sensors, with the intended application being close range aerial photogrammetry. Other methods of 3D point cloud generation such as structure from motion do not require the parameters of exterior orientation (i.e. the position and orientation) of the camera station as well as the 3D coordinates of several ground control points to be known prior to scene reconstruction $[6,7]$. Contrary to this, in order to obtain the 3D coordinates of a point within a surveyed scene, photogrammetry requires that the parameters of exterior of orientation as well as the coordinates of several ground control points be known or determined prior to scene reconstruction [6]. It is for this reason that the following thesis will focus on applications geared towards direct georeferencing for closed range aerial photogrammetry.

In order to obtain the required data to calculate the exterior orientation parameters during flight, most direct georeferenced systems synchronize the digitallycaptured image times with a data logger or telemetry log files, which are analyzed during post-processing $[5,8]$. This requires the use of a highly-accurate system as

any discrepancies will have a direct impact on the results obtained [4]. The objective 
of this research is not to conduct an aerodynamic or an electronic analysis on the proposed system but to improve on the already available methods and techniques for direct georeferencing through added onboard computer functionality. This is done by incorporating a Raspberry Pi 3B into the system to enable communication with the autopilot/flight controller. Once this two way communication was established, each time an image was captured during flight, the telemetry data could be pulled from the autopilot and associated directly to the image. Finally, the captured georeferenced images were used to produce dense points clouds and were compared to values obtained through indirect georeferencing.

\subsection{Thesis Outline}

Chapter 2 presents a literature review on the topics of aerial photogrammetry, UAV flight planning and the principles of georeferencing. Furthermore, some of the relevant research currently being done in the field of direct georeferencing is discussed.

In Chapter 3, a detailed explanation of the experimental methodology is presented. It includes a complete overview of the UAV and data acquisition system that was implemented for this research, including all additional hardware and software used during the experimentation. Moreover, the approach used for all tests in this thesis is presented.

Chapter 4 summarizes the results for all tests and includes a discussion on the results. Lastly, Chapter 5 presents the conclusions drawn from this thesis and provides recommendations for future work that could be conducted based on the work presented in this thesis. 


\section{Chapter 2}

\section{Literature Review}

\subsection{Mathematical Background}

In this section, the mathematical principles and well as the conventions that will be used for all calculations relating to the photogrammetric process throughout this thesis are presented and explained.

\subsubsection{Description of Position, Orientation and Frame}

Given a coordinate system, $\{A\}$, any point located in that space may be described using a $3 \times 1$ position vector, ${ }^{A} P[9]$. This is illustrated in Figure 2.1. 


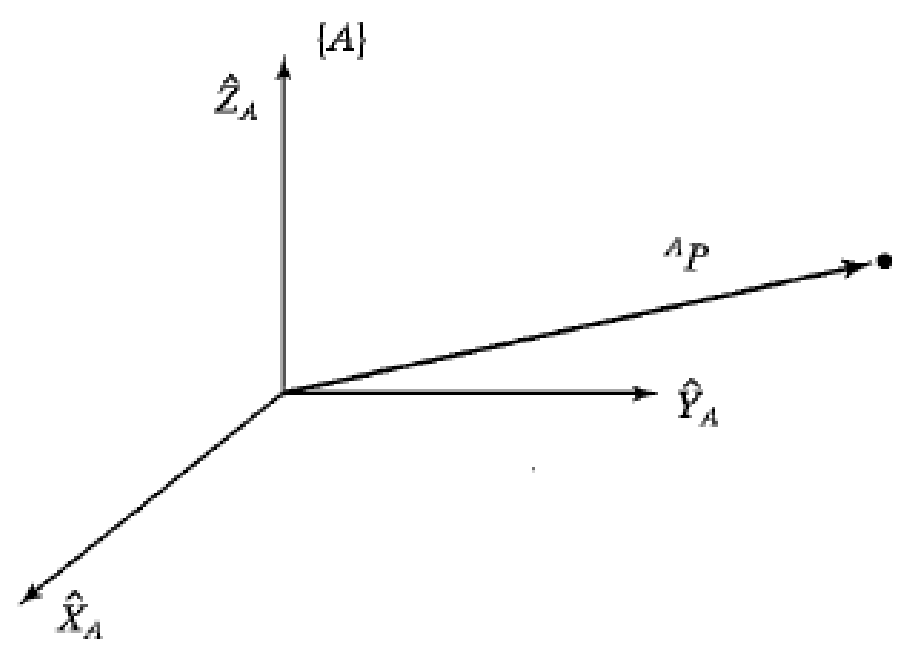

Figure 2.1: Vector shown relative to frame $\{A\}[9]$.

The variables $\hat{X}_{A}, \hat{Y}_{A}$ and $\hat{Z}_{A}$ shown in Figure 2.1 correspond to the unit vectors of coordinate system $\{\mathrm{A}\}$. When dealing with the transformation of coordinates, there are often multiple other reference systems which are defined on top of the initial coordinate system. Therefore all vectors should contain some information which identifies the coordinate system to which it belongs.

$$
{ }^{A} P=\left[\begin{array}{c}
P_{x} \\
P_{y} \\
P_{z}
\end{array}\right]
$$

In the case of Equation 2.1, the vector $P$ whose components are $P_{x}, P_{y}$ and $P_{z}$ are located in the reference system $\{A\}$. To describe the orientation of a body, initially a coordinate system is associated to the body after which a description of the coordinate system relative to the reference system is given [9]. Figure 2.2 illustrates an example where the coordinate system $\{B\}$ has been associated in a known way. In order to 


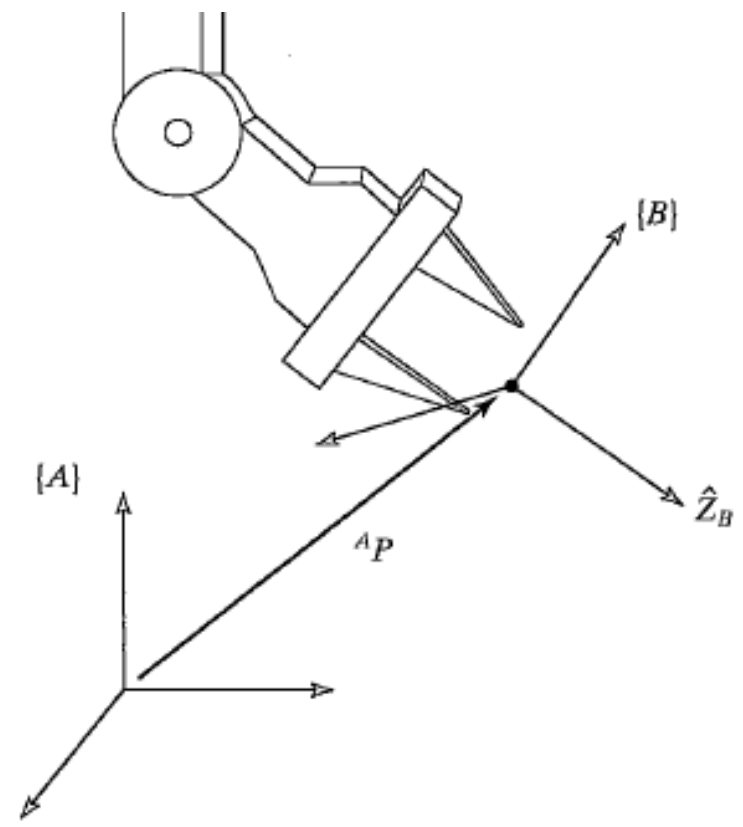

Figure 2.2: Position and orientation [9].

get the orientation of the body, simply providing a description of $\{B\}$ relative to $\{A\}$ is sufficient [9]. Alternatively, writing the unit vectors of the 3 principals axes of a coordinate system in terms of another presents a different manner to represent orientation. For the example shown in Figure 2.2, the unit vectors of $\{B\}$ are $\hat{X}_{B}$, $\hat{Y}_{B}$ and $\hat{Z}_{B}$. Once they have been written relative to $\{A\}$, they will be denoted as ${ }^{A} \hat{X}_{B},{ }^{A} \hat{Y}_{B}$ and ${ }^{A} \hat{Z}_{B}$ each being a $3 \times 1$ vector. For convenience these three column vectors are grouped together to form a single $3 \times 3$ matrix [9]. The former is referred to as a rotation matrix, $\mathbf{R}_{B}^{A}$. To summarize [9]:

$$
\mathbf{R}_{B}^{A}=\left[\begin{array}{lll}
{ }^{A} \hat{X}_{B} & { }^{A} \hat{Y}_{B} & { }^{A} \hat{Z}_{B}
\end{array}\right]=\left[\begin{array}{lll}
r_{11} & r_{12} & r_{13} \\
r_{21} & r_{22} & r_{23} \\
r_{31} & r_{32} & r_{33}
\end{array}\right]
$$


Lastly, in order to describe a frame, a set of four vectors which corresponds to the orientation (combination of three $3 \times 1$ vectors which form the rotation matrix) and a postion vector are required. They are represented as a pair, which is shown in Equation 2.3.

$$
\{B\}=\left\{\mathbf{R}_{B}^{A},{ }^{A} P_{B O R G}\right\}
$$

Where $P_{B O R G}$ is a vector which connects the origin of both coordinate systems.

\subsection{UAVs for Aerial Surveying}

Uninhabited aerial vehicles, which are frequently referred to as: drones, unmanned vehicle systems (UVS), remotely piloted vehicles (RPVs), remotely piloted aircraft systems (RPAS) and micro aerial vehicles (MAVs), are defined as: "a generic aircraft designed to operate with no human pilot onboard [10,11]". A UAV system is comprised of four main parts; an aircraft component, various sensors, payloads and a ground control station [11]. UVS International [12] outlines three main categories in which UAVs are grouped, each of which contain multiple sub-categories. The former are $[3,10]$ :

- Tactical UAVs

- Strategic UAVs

- Special task UAVs

Tactical UAVs encompass a multitude of different systems, which are listed in Table 
2.1 along with their defining characteristics.

Table 2.1: Sub-categories of tactical UAVs. Based on table in [13]

\begin{tabular}{|c|c|c|c|c|}
\hline Tactical UAVs Sub-categories & Acronym & Max high [m] & Autonomy [hours] & Weight [Kg] \\
\hline Micro & mew & 250 & 1 & 5 \\
\hline Mini & Mini & $150-300$ & 2 & 150 \\
\hline Close Range & CR & 3000 & $2-4$ & 150 \\
\hline Short Range & SR & 3000 & $3-6$ & 200 \\
\hline Medium Range & MR & 5000 & $6-10$ & 1250 \\
\hline Medium Range Endurance & MRE & 8000 & $10-18$ & 1250 \\
\hline Low Altitude Deep Penetration & LADP & $50-9000$ & $0.5-1$ & 350 \\
\hline Low Altitude Long Endurance & LALE & 3000 & 24 & 30 \\
\hline Medium Altitude Long Endurance & MALE & 14000 & $25-48$ & 15000 \\
\hline
\end{tabular}

Strategic UAVs have an endurance which ranges from two to four days and operates at altitudes exceeding $20000 \mathrm{~m}$ and include the sub-categories high-altitude long-endurance UAVs [10]. Finally, Special Task UAVs are systems that are designed for very specific task such as the case with unmanned autonomous combat vehicles employed in military missions [10]. Initially, UAV systems and platforms were designed for military purposes with their intended objectives being for surveillance, mapping and reconnaissance in hostile territories without the risk of endangering the lives of soldiers $[3,10]$. However, their use in many civil applications have become common due to the ability to conduct rapid surveys at low costs while also being able to map and survey areas that would otherwise be inaccessible or have been deemed too dangerous for traditional surveying techniques [3]. The use of UAVs for remote sensing and mapping applications has been discussed in detail in the following articles $[3,10,13-15]$ while the preparation and processing of the acquired data is discussed in [16-20]. Moreover, Table 2.2 lists the different applications in which UAV images are currently being used. 
Table 2.2: Summary of the various applications of UAVs in the field of geomatics [10]

\begin{tabular}{|c|c|}
\hline Applications & Details \\
\hline Agriculture & $\begin{array}{l}\text { - Obtain quick and accurate record of damages } \\
\text { - Identify potential problems in the field or with crops }\end{array}$ \\
\hline Archeology & 3D surveying and mapping of sites and structures \\
\hline Emergency Management & $\begin{array}{l}\text { - Acquire images for early impact assessments and } \\
\text { rescue planning } \\
\text { - Flights can be performed over contaminated areas } \\
\text { without any dangers for the operators }\end{array}$ \\
\hline Environment & $\begin{array}{l}\text { - Monitor land and water at multiple epochs } \\
\text { - Road mapping } \\
\text { - Excavation volume computations } \\
\text { - Volcano monitoring } \\
\text { - Natural resource documentations for geological analyses }\end{array}$ \\
\hline Forestry & $\begin{array}{l}\text { - Assessments of woodlots } \\
\text { - Fire surveillance } \\
\text { - Vegetation monitoring } \\
\text { - Species identification }\end{array}$ \\
\hline Traffic Management & $\begin{array}{l}\text { - Surveillance } \\
\text { - Travel time estimation } \\
\text { - Lane occupancy } \\
\text { - Incidence response }\end{array}$ \\
\hline
\end{tabular}

For applications in the fields of architecture and archeology, UAV photogrammetry is used to survey and map historical sites and structures and are used to generate 3D point clouds and digital terrain models. The use of UAVs for these types of applications presents a viable alternative to more expensive and time consuming techniques such as traditional or manned aircraft photogrammetry [10]. The typical 
aerial surveying workflow was shown in Figure 1.2 of Section 1.1 and consists primarily of the following operations: flight/mission planning, data/image acquisition, image orientation and 3D point cloud and DTM generation $[3,10]$.

The first step in the aerial surveying process pipeline is flight planning which encompasses: identifying the mission objectives, familiarising oneself with the area of interest and determining flight parameters as well as the camera's intrinsic parameters. Setting and identifying the mission objectives is defining what is going to be accomplished during the flight as well as what type of data is going to be collected. Next, the area of interest should be examined in order to identify any potential obstacles that could hinder the flight.

Once the first two steps are completed, the flight parameters must be calculated. Firstly, using the required image scale and camera focal length it is possible to determine the mission's flying height. This is done using Equation 2.4 [21]

$$
m_{b}=\frac{H}{c}
$$

where $m_{b}$ is the photo scale number, $H$ is the mission's flying height and $c$ is the camera's focal length. The ground sampling distance (GSD) can then be calculated using equation $2.5[22]$.

$$
G S D=\frac{H}{c} \times d
$$

with $d$ being the size of a pixel. A schematic of the definition of the GSD is shown in Figure 2.3. 


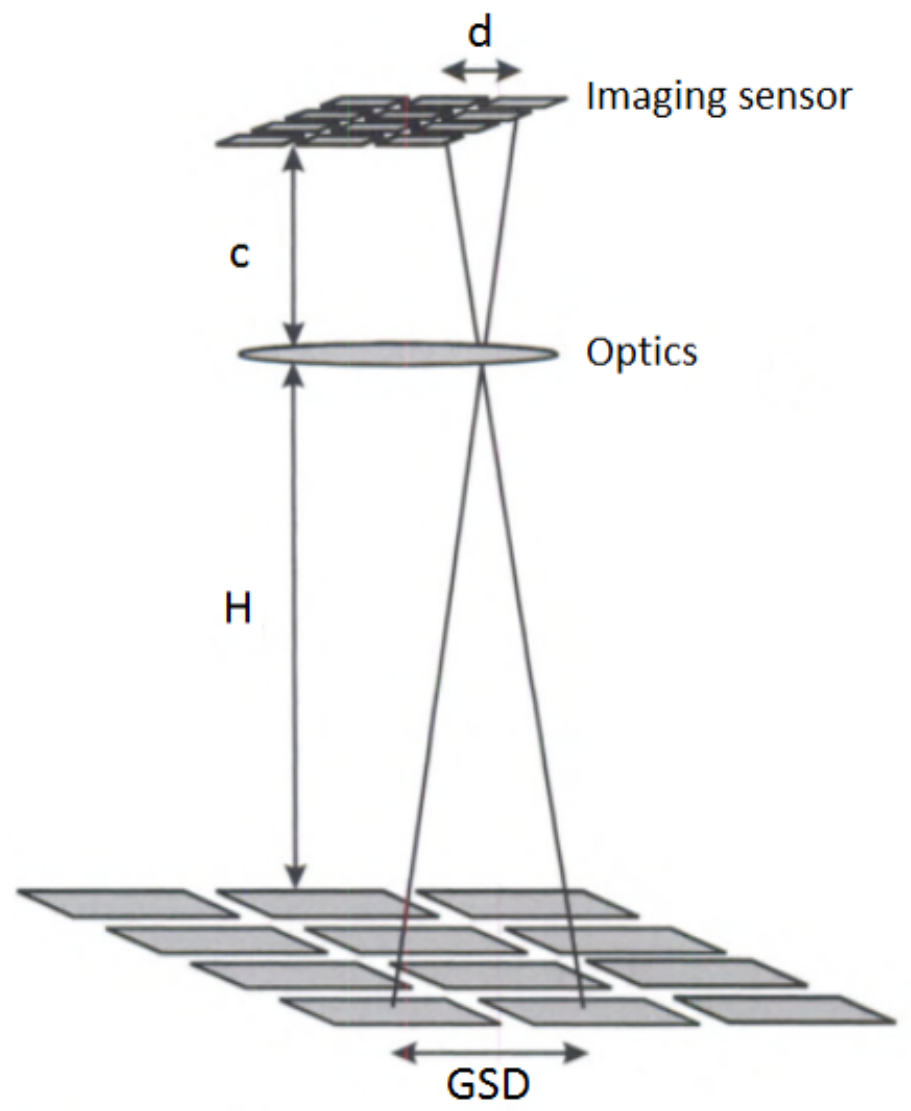

Figure 2.3: Ground sampling distance [23]

When the mission's objective is to produce detailed 3D models from the captured images, small GSDs are required therefore low-altitude flights are needed with large overlaps [10]. Another useful parameter to calculate is the ground area of one photograph, which is determined using Equation 2.6 [21].

$$
F_{b}=S^{2}=s^{2} m_{b}^{2}
$$

When capturing images for aerial surveys it is important to have a certain percentage of overlap between adjacent images for aerial triangulation. Figure 2.4 
provides a visual representation of the overlap between images for three cases; manual flight, low cost navigation system assisted flight and high precision automated flight and image acquisition [10].
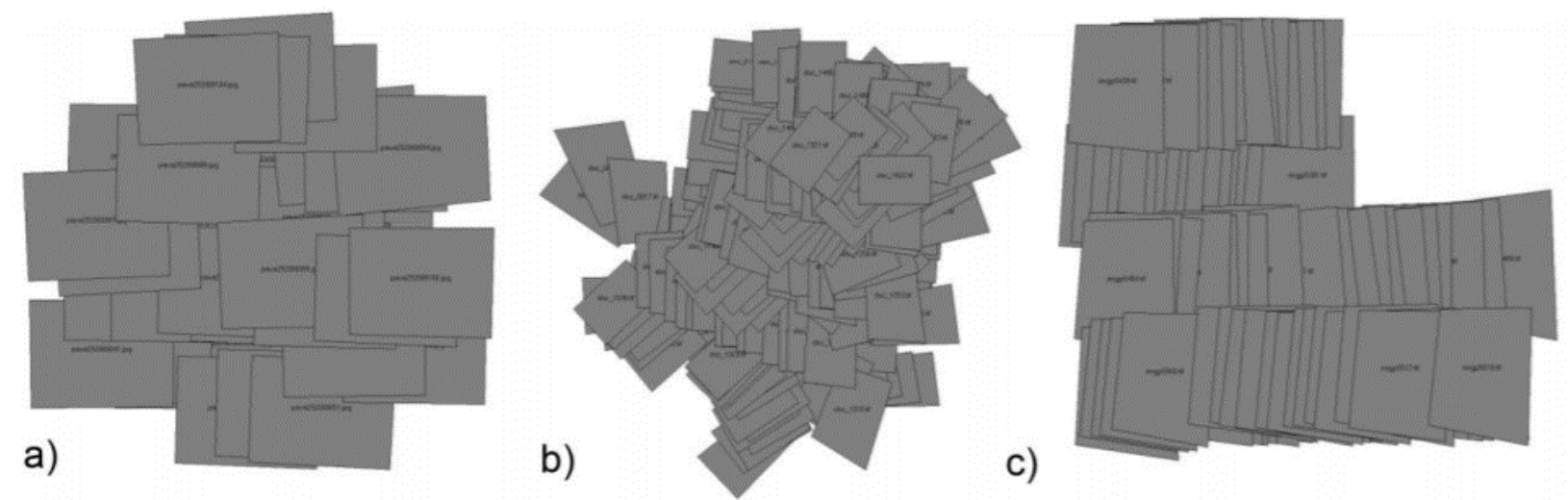

Figure 2.4: a) manual flight; b) low cost navigation system assisted flight; c) high precision automated flight and image acquisition [10].

The baselength for $l$ of forward overlap as well as the distance between flight lines for $q$ amount of side overlap are given by Equations 2.7 and 2.8 respectively.

$$
\begin{aligned}
& B=S\left(1-\frac{l}{100}\right) \\
& A=S\left(1-\frac{q}{100}\right)
\end{aligned}
$$

where $S$ is obtained using Equation 2.9

$$
S=s m_{b}
$$

Image acquisition, orientation as well as 3D model generation are addressed in detail 
in the next section of this chapter.

\subsection{Principles of Georeferencing}

\subsubsection{The Collinearity Equation}

The collinearity equations define a mathematical model which relate the image coordinates to the object coordinate system. Figure 2.5 shows the relationship between the image and object coordinate systems.

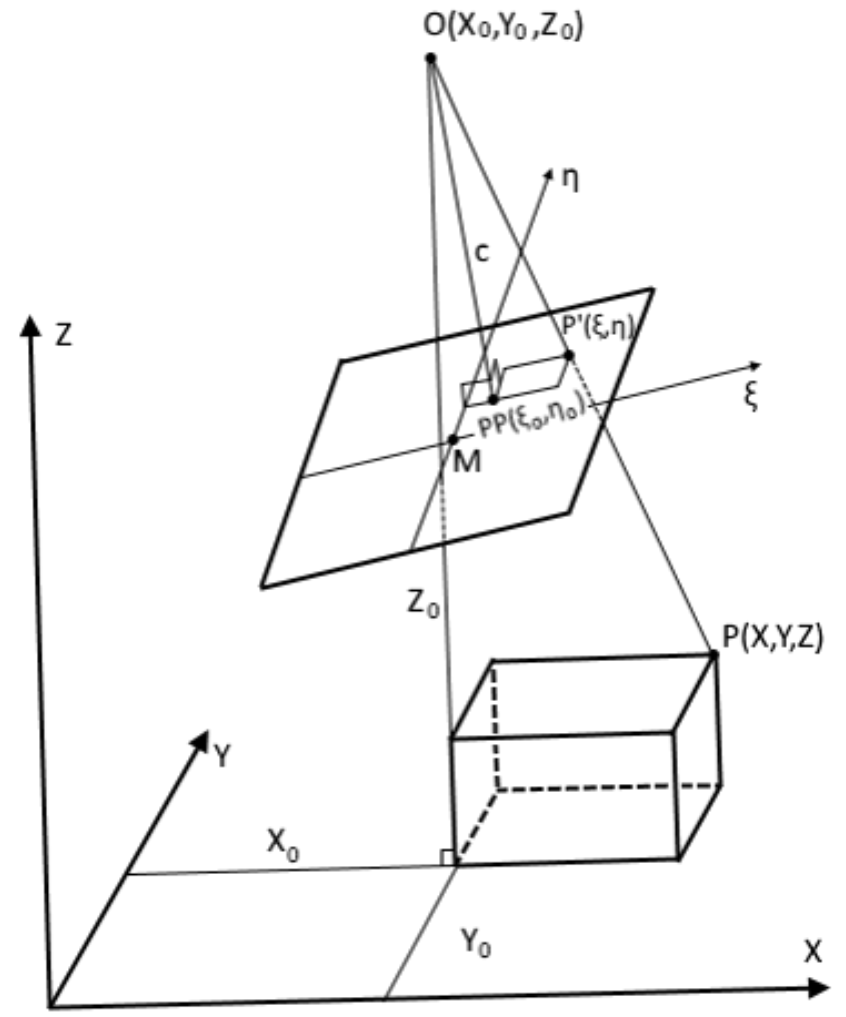

Figure 2.5: Relation between image and object plane [9].

There are two coordinate systems to be considered. The first is the $X Y Z$ system, which is used to describe a point located in the object space, sometimes called 
world or global space, denoted by $P$ in Figure 2.5. The second is the $\xi \eta$ system, which is used to describe the projection of an object point on to the image plane, denoted by $P^{\prime}$ in Figure 2.5. The other important elements which need to be considered when describing the collinearity equation is the object coordinates of the camera station, which is marked as point $O$ in Figure 2.5 and whose coordinates are $\left(X_{0}, Y_{0}, Z_{0}\right)$. Furthermore, the interior orientation parameters of the camera must be known. The elements of interior orientation refer to the principal distance (also called the effective focal length), $c$, as well as the principal point, $P P$, whose image coordinates are represented as $\left(\xi_{0}, \eta_{0}\right)$ in Figure 2.5. Normally, the interior orientation parameters of the camera are provided by the manufacturer or determined via calibration conducted in a laboratory as they are required and assumed to be known values for the bundle adjustment process $[24,25]$. It is important to note that the orientation of the image plane may not always be aligned with that of the object plane, therefore the coordinate system of the object plane would need to be transformed using a transformation matrix. Since the planes are not required to be coincident, there is no translation component to the transformation thus using a rotation matrix which describes the rotations about the $X, Y$ and $Z$ axis is sufficient. Figure 2.6 illustrates the aforementioned case. 


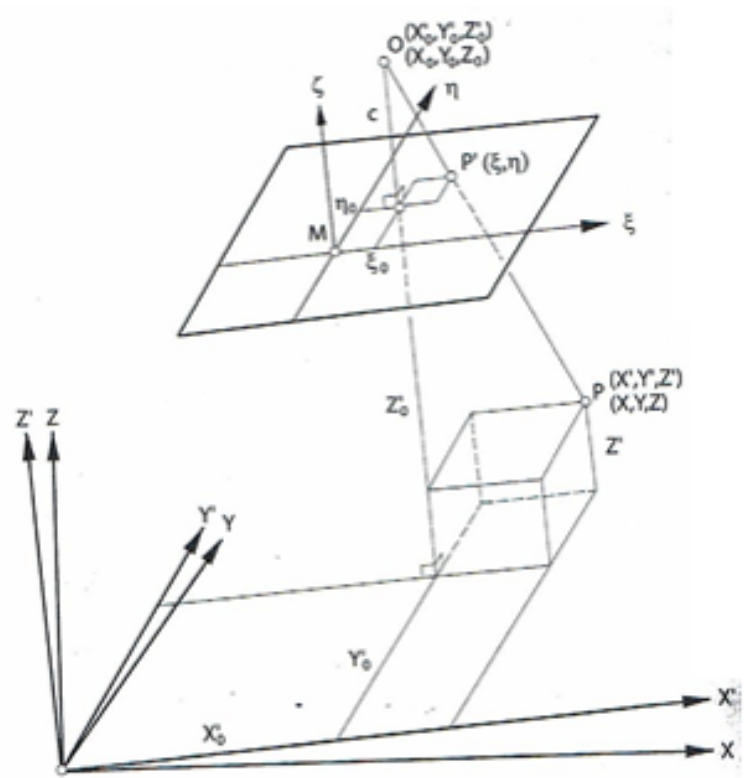

Figure 2.6: Collinearity model of point $\mathrm{P}$ projected on to the image plane [21]

Equating the length of the projected surface on the image plane with its counterpart in the object space for all axes from Figure 2.6 leads to a system of two equations [21].

$$
\begin{gathered}
\frac{\xi-\xi_{0}}{c}=\frac{X^{\prime}-X_{0}^{\prime}}{Z_{0}^{\prime}-Z^{\prime}} \\
\frac{\eta-\eta_{0}}{c}=\frac{Y^{\prime}-Y_{0}^{\prime}}{Z_{0}^{\prime}-Z^{\prime}}
\end{gathered}
$$

An expression for the image coordinates of an object point projected on the images plane can be expressed by isolating $\xi$ and $\eta$ from Equation 2.10 and 2.11 respectively.

$$
\xi=\xi_{0}-c \frac{X^{\prime}-X_{0}^{\prime}}{Z^{\prime}-Z_{0}^{\prime}}
$$




$$
\eta=\eta_{0}-c \frac{Y^{\prime}-Y_{0}^{\prime}}{Z^{\prime}-Z_{0}^{\prime}}
$$

As previously stated, there are occasions when the coordinate systems of the object space do not align with that of the image plane which requires the use of a transformation matrix to properly align the coordinate systems. To perform this transformation, the coordinates of the initial system (in object space) are multiplied by a $3 \times 3$ rotation matrix $\mathbf{R}[21,26]$.

$$
\left[\begin{array}{l}
X-X_{0} \\
Y-Y_{0} \\
Z-Z_{0}
\end{array}\right]=\left[\begin{array}{lll}
r_{11} & r_{12} & r_{13} \\
r_{21} & r_{22} & r_{23} \\
r_{31} & r_{32} & r_{33}
\end{array}\right]\left[\begin{array}{l}
X^{\prime}-X_{0}^{\prime} \\
Y^{\prime}-Y_{0}^{\prime} \\
Z^{\prime}-Z_{0}^{\prime}
\end{array}\right]
$$

Then by multiplying Equations 2.12 and 2.13 by $\mathbf{R}^{-1}$, which corresponds to the inverse of the rotation matrix and substituting the resulting expressions into Equation 2.14 an expression demonstrating the relation between the coordinates of a point in the image plane and that of its corresponding point in the object space can be determined by isolating $\xi$ and $\eta[21]$.

$$
\begin{gathered}
\xi=\xi_{0}-c \frac{r_{11}\left(X-X_{0}\right)+r_{21}\left(Y-Y_{0}\right)+r_{31}\left(Z-Z_{0}\right)}{r_{12}\left(X-X_{0}\right)+r_{23}\left(Y-Y_{0}\right)+r_{33}\left(Z-Z_{0}\right)} \\
\eta=\eta_{0}-c \frac{r_{12}\left(X-X_{0}\right)+r_{22}\left(Y-Y_{0}\right)+r_{32}\left(Z-Z_{0}\right)}{r_{13}\left(X-X_{0}\right)+r_{23}\left(Y-Y_{0}\right)+r_{33}\left(Z-Z_{0}\right)}
\end{gathered}
$$

In order to obtain the coordinates of the point of interest in object space, the 
variables $X$ and $Y$ can be isolated from Equations 2.15 and 2.16. However, since there are two equations and three unknowns $(X, Y$ and $Z)$ the resulting equations must be written as a functions of $Z[21]$.

$$
\begin{aligned}
& X=X_{0}+\left(Z-Z_{0}\right)\left(\frac{\left.r_{11}\left(\xi-\xi_{0}\right)+r_{12}\left(\eta-\eta_{0}\right)+r_{13} c\right)}{\left.r_{31}\left(\xi-\xi_{0}\right)+r_{32}\left(\eta-\eta_{0}\right)+r_{33} c\right)}\right) \\
& Y=Y_{0}+\left(Z-Z_{0}\right)\left(\frac{\left.r_{21}\left(\xi-\xi_{0}\right)+r_{22}\left(\eta-\eta_{0}\right)+r_{23} c\right)}{\left.r_{31}\left(\xi-\xi_{0}\right)+r_{32}\left(\eta-\eta_{0}\right)+r_{33} c\right)}\right)
\end{aligned}
$$

When applying Equations 2.15 through 2.18, it is assumed that the following parameters are known; the elements of interior orientation which are camera specific. Moreover, the elements of exterior orientation must be known. The different methods for determining the elements of exterior orientation will be discussed in the later sections of this chapter. Observing Equations 2.15 through 2.18, it can be seen that for each point in object space, there is a single corresponding image point. However, Equations 2.17 and 2.18 are written as a function of $Z$, which leads to the conclusions that for each point there is an infinite amount of possible corresponding object points. The former is the reason that it is impossible to determine the coordinates and reconstruct an object in 3D based on a single image [21]. Therefore, a second image of the object taken from a different view or distance must be obtained and then solving the collinearity equations for both images in tandem will allow for the full three-dimensional coordinates of the point of interest to be determined. With enough adjacent images, it is then possible to produce a complete three-dimensional model of the object or structure being studied and to obtain physical measurements from said model. 


\subsubsection{Indirect Method}

Prior to the technological developments of new systems of three-dimensional data acquisition which led to the use of new sensors whose direct orientation could be determined using GPS and inertial navigation system (INS), indirect image orientation was the favored approach for the orientation of image based frame sensors such as cameras. This method treats the exterior orientation parameters of the cameras perspective center for each captured image as an unknown which needs to be determined and is estimated using a bundle adjustment process together with aerial triangulation $[4,24]$. If there are no additional orientation sensors used during a mission or there are only rough estimates of the exterior orientation parameters of the imaging sensor, then the method that was described above presents the only means by which the exterior orientation parameters can be determined [24].

Using the indirect method, the six exterior orientation parameters $(X, Y, Z, \omega$, $\phi, \kappa)$ are determined using the mathematical model which relates the image and object spaces (i.e the collinearity equations) by defining the correlation between several ground control points and their respective image coordinates [25]. In the case where multiple or a series of adjacent images are being evaluated, the orientation is solved by aerial triangulation [24], which is a basic method commonly employed in the analysis of aerial images with the objective of calculating the three-dimensional coordinates of object points as well as the exterior orientation parameters of images [27]. Image coordinates are related to the object coordinate system using the collinearity equation assuming the standard model of central perspective is being employed [4]. Ground control points are defined as an object point which is represented in the image and at which the actual three-dimensional object (terrain) coordinates are known [28]. An example of a targeted ground control point is shown in Figure 2.7. 


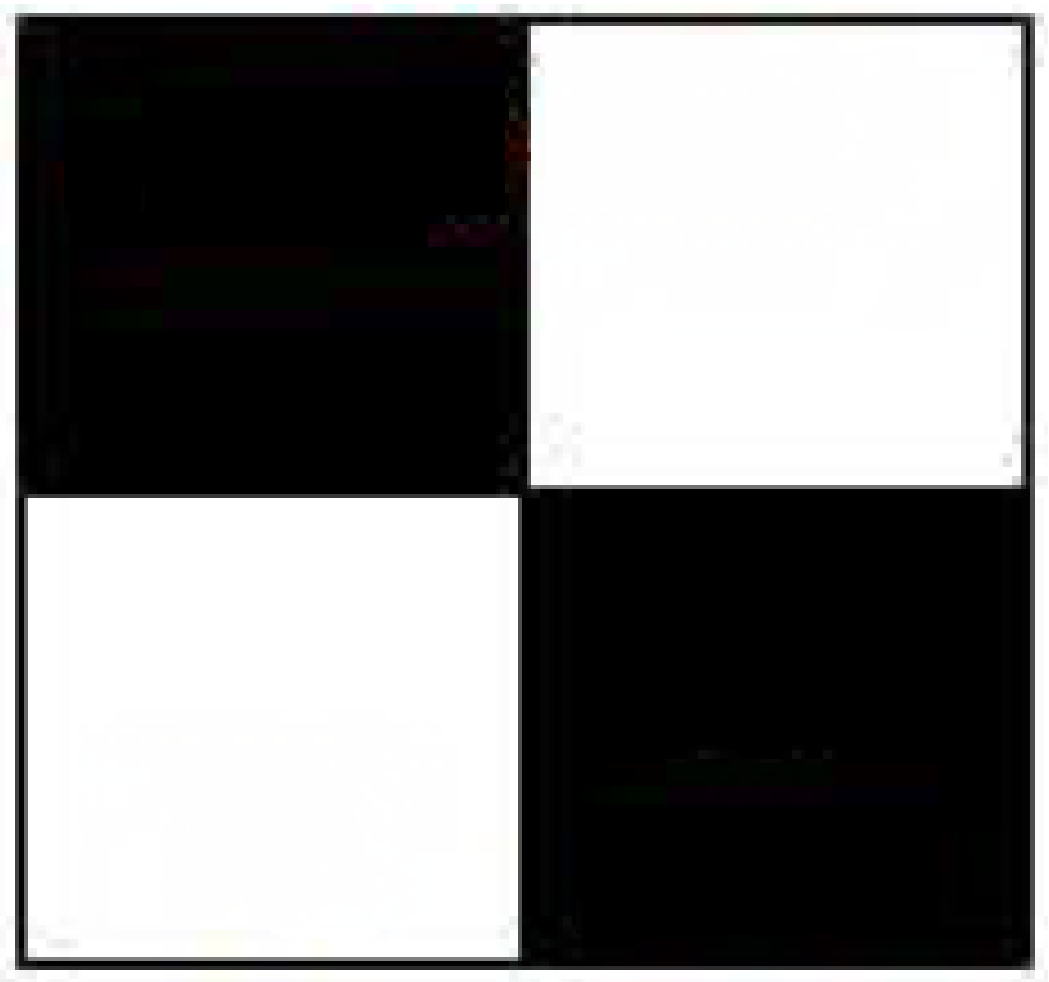

Figure 2.7: Targeted ground control point.

There exist two types of ground control points, targeted and natural control points. Prior to starting a survey, certain points within the area of interest are identified by placing a target whose coordinates are known. The other option would be to determine the coordinates of distinct and identifiable points such as unique building or terrain features which could be easily and repeatedly identified in an image, these are called natural control points [28]. There are a set of basic rules and guidelines for the use of control points in photogrammetry provided in [28], the three most important guidelines that were identified were:

1. There should be a minimum of three control points for each image. However, in the case of aerial surveying where the altitude remains constant, two full control points are sufficient.

2. The three control points should never be placed in a straight line. Instead, they 
should be placed in a triangle formation.

3. To incorporate as many ground control points as possible, within reason.

It is important to note that the best accuracy of the results will be in the areas immediately surrounding the control points. Figure 2.8 illustrates the case of determining the exterior orientation with unknown parameters given two separate images.

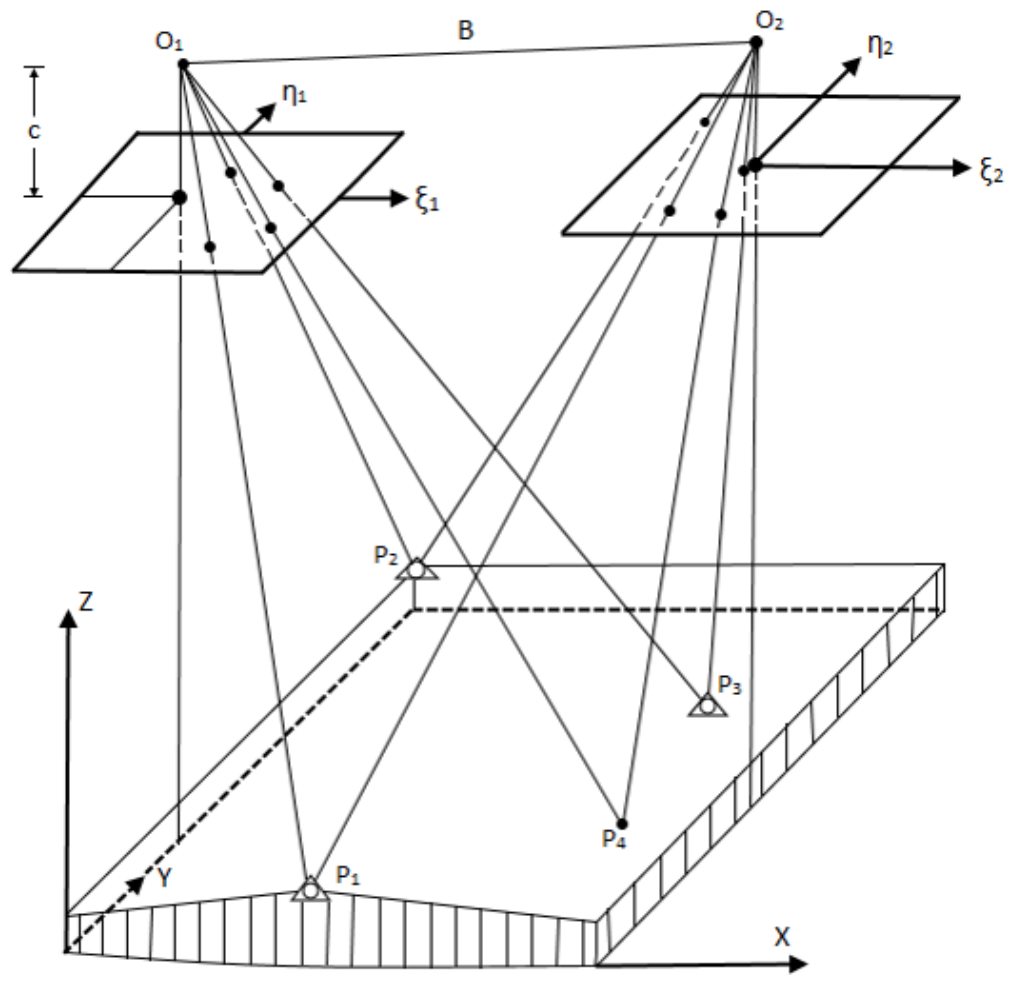

Figure 2.8: Relation between image and object plane [28].

The exterior orientation parameters of both images shown in Figure 2.7 are unknown therefore there are six variables that need to be determined per image $\left(X_{01}\right.$, $\left.Y_{01}, Z_{01}, \omega_{01}, \phi_{01}, \kappa_{01}\right)$ and $\left(X_{02}, Y_{02}, Z_{02}, \omega_{02}, \phi_{02}, \kappa_{02}\right)$. As previously stated, there should be multiple control points associated to each image. For the case shown 
in Figure 2.7, there are three control points that appear in each image therefore by applying Equations 2.15 and 2.16 to each image, a series of six equations which describe the position of the ground control point in the image plane can be produced. The former can be visualized as follows [21]:

$$
\begin{aligned}
\xi_{i} & =f\left(\xi_{0}, c, X_{0}, Y_{0}, Z_{0}, \omega, \phi, \kappa, X_{i}, Y_{i}, Z_{i}\right) \\
\eta_{i} & =f\left(\eta_{0}, c, X_{0}, Y_{0}, Z_{0}, \omega, \phi, \kappa, X_{i}, Y_{i}, Z_{i}\right)
\end{aligned}
$$

for $i=1,2,3$. Since there are now six equations and six unknowns per image, it is possible to obtain values for the exterior orientation of each image by solving the system of equations.

\subsubsection{Direct Method}

The topic of direct georeferencing involves calculating the exterior orientation parameters $(X, Y, Z, \omega, \phi, \kappa)$ using the data from the onboard sensors of an aircraft, specifically GPS/INS and inertial measurement sensors. This topic is thoroughly addressed in the following articles $[5,8,24,25,29-32]$. The direct measurement of the exterior orientation parameters of any sensor during data recording with sufficient accuracy became possible with the development and availability of integrated GPS/inertial systems implemented on commercially available UAVs [24,30]. The ability to directly determine the exterior orientation parameters of the camera station at any instant in time is the fundamental difference between the direct method of georeferencing and indirect method that was presented in the previous section. The required accuracy that a system must achieve for the position and attitude will depend on the given application. The former is mainly defined by the mapping scale [25]. However, using 
current commercially available direct georeferencing systems (UAVs and sensors), it is possible to achieve an acceptable accuracy for a multitude of mapping scenarios and applications [30]. Figure 2.9 provides a visual representation of the cost associated with each step in the process of traditional photogrammetry and compares it to the alternative direct georeferencing approach.

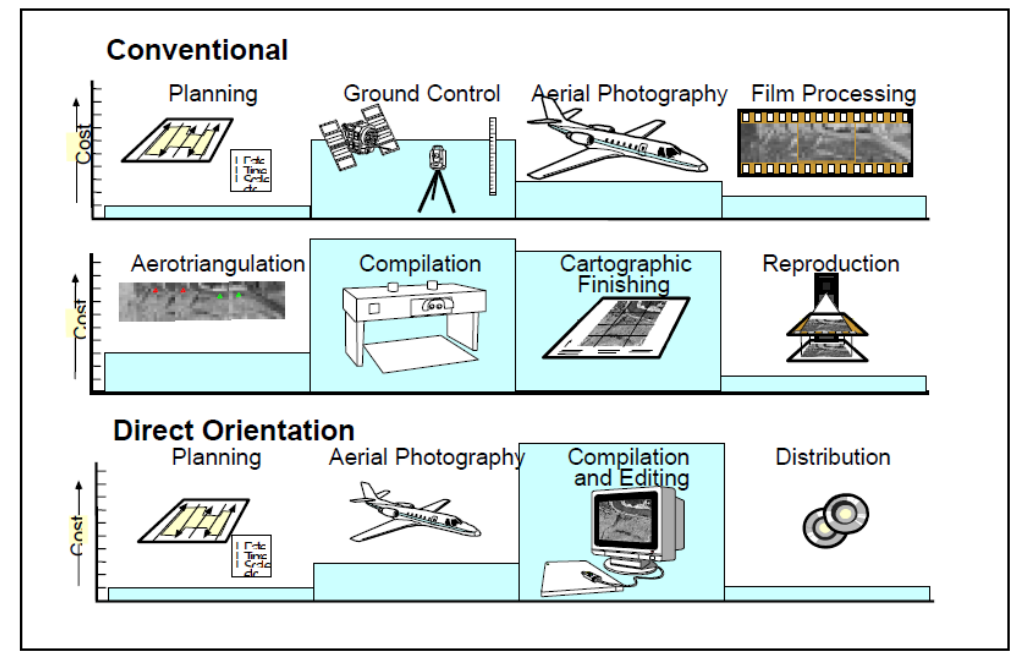

Figure 2.9: Flow chart comparing traditional and direct georeferenced photogrammetry methods $[30,33]$

Observing the figure it can be seen that the direct georeferencing method requires less steps and can be carried out at a lower costs compared to its counterpart, the traditional method. The traditional method of photogrammetry is a commonly used approach; however, it requires additional steps in both the preparation of the survey and the post-processing of the results which are not required in the direct approach. As explained in Section 2.3.2, the conventional method uses indirect georeferencing, which requires the use of ground control points in order to determine the exterior orientation parameters. Placing and subsequently measuring the positions of each individual ground control points, using either a GPS system or total station requires time, which increases costs. However, as previously stated, the availability of quality GPS/INS systems for commercial UAVs has allowed for high-accuracy 
and real-time position and orientation information to be obtained during flight. This data can eliminate the need for the aerial triangulation process and allows for a lower number of ground control points to be used in the survey process which leads to an overall reduction in the aerial surveying/mapping costs $[24,25,34]$. Moreover, it has even been suggested that the development of precise direct georeferencing systems could lead to the elimination of ground control points for surveying and mapping applications [34]. Another important aspect to consider when comparing both methods is the post-processing of the data and results. In both cases, there is a certain amount of interactive editing that is required in order to obtain results [24]. However, as observed in Figure 2.9 this process is more involved for traditional photogrammetry. Furthermore, during the aerotriangulation step of the workflow, the exterior orientation must be calculated for each image which increases the post-processing time. These additional steps add both to the cost and the time required to acquire and process the data.

The approach that is taken to solve direct georeferencing problems is shown in Figure 2.10 and is discussed in $[4,30,32,33,35,36]$. The problem is broken down into a series of vector summations and coordinate transformations in order to use the GPS/INS information from the aircraft to determine the coordinates of the point of interest in the mapping plane.

Articles $[30,33]$ provide an equation to obtain the coordinates of a vector point $i$ in the mapping frame as:

$$
{ }^{m} r_{i}={ }_{g p s}^{m} r(t)+\mathbf{R}_{b}^{m}\left(S_{i} \mathbf{R}_{c}^{b} r_{i}^{c}+r^{c}{ }_{i n s}-r^{g p s}{ }_{i n s}\right)
$$

where [30]: 


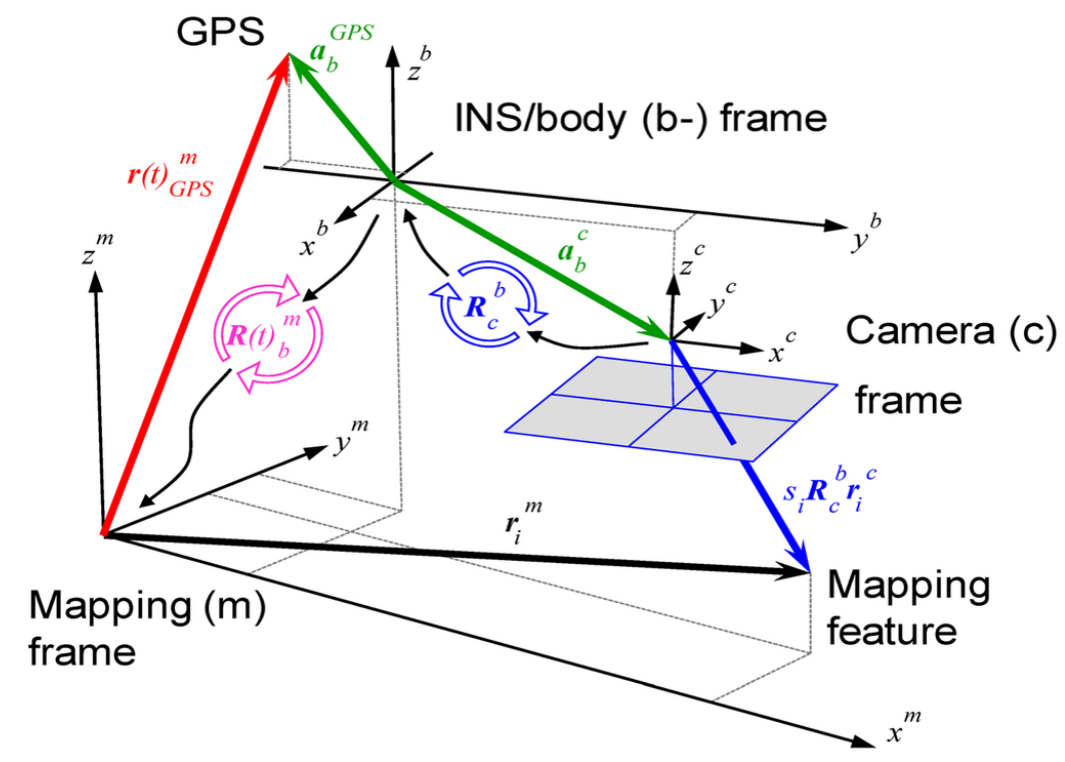

Figure 2.10: Illustration of the general notion of direct georeferencing [30].

- ${ }^{m} r_{i}=$ coordinate of a vector point $i$ in the mapping frame (denoted by $m$ in Figure 2.10)

- ${ }_{g p s}^{m} r(t)=$ coordinate vector of the navigation sensors (GPS/INS) in the $m$-frame

- $S_{i}=$ scale factor

- $\mathbf{R}_{b}^{m}$ is the rotation matrix between the navigation sensors' body frame (b-frame in Figure 2.10) and the mapping frame at a given time of exposure, $t$

- $r^{c}{ }_{i n s}$ is the vector between the inertial measurement unit's center and the camera's principal point

- $r^{g p s}{ }_{i n s}$ is the vector between the inertial measurement unit's center and the GPS antenna center

Where $\mathbf{R}_{c}^{b}$ is the physical offset angles between the inertial measurement unit and the digital camera, shown in Figure 2.11 and is referred to as the boresight $[33,35,37]$. The boresight represents the misalignment between the camera and body frames. 


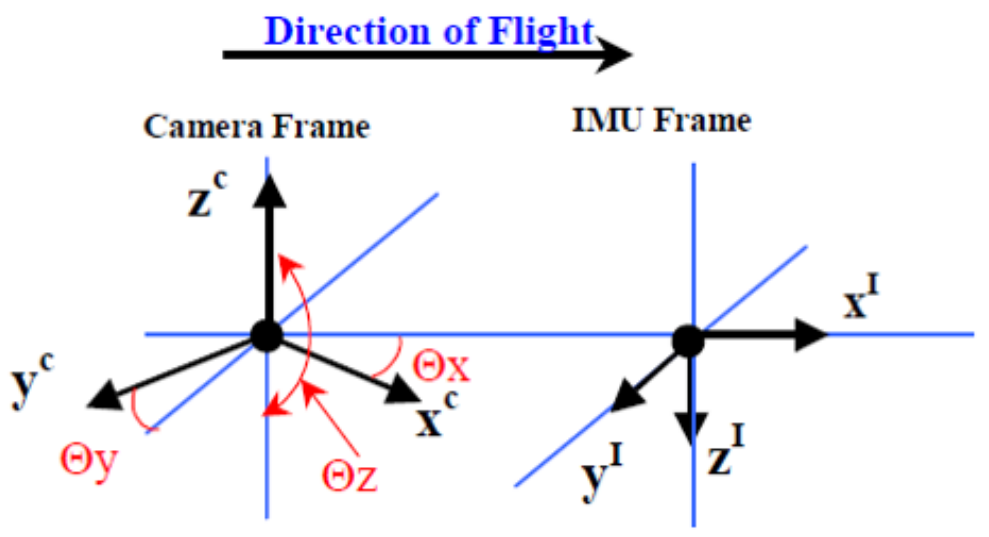

Figure 2.11: Visual representation of the misalignment between the camera and body frames [35].

The rotation matrix is obtained by performing a rotation about the $X, Y$ and $Z$ axes of the body frame and it is assumed that the offset angles $\left(\theta_{X}, \theta_{Y}\right.$ and $\left.\theta_{Z}\right)$ remain constant throughout the flight, meaning that the camera is mounted in a fixed position on the aircraft and is unable to freely change its orientation during flight. Equation 2.21 is used to determine $\mathbf{R}_{c}^{b}$ [35].

$$
\mathbf{R}_{c}^{b}=\left[\begin{array}{ccc}
1 & 0 & 0 \\
0 & \cos \theta_{X} & \sin \theta_{X} \\
0 & -\sin \theta_{X} & \cos \theta_{X}
\end{array}\right]\left[\begin{array}{ccc}
\cos \theta_{Y} & 0 & -\sin \theta_{Y} \\
0 & 1 & 0 \\
\sin \theta_{Y} & 0 & \cos \theta_{Y}
\end{array}\right]\left[\begin{array}{ccc}
\cos \theta_{Z} & \sin \theta_{Z} & 0 \\
-\sin \theta_{Z} & \cos \theta_{Z} & 0 \\
0 & 0 & 1
\end{array}\right]
$$

It is important to note that the misalignment angles of the boresight cannot be measured or determined directly; however, there are two methods that can be used to approximate their value. The first method involves comparing the derived angles of the GPS/IMU derived angles with the values which were calculated using aerial triangulation $[4,36,37]$. The difference between the two series of values can then be 
assumed as the three components of the boresight misalignment angles. The second method involves treating the three unknown angles as additional parameters in the GPS/IMU assisted bundle adjustment [4,35].

The limitations and challenges that arise in direct georeferencing systems are discussed in $[4,33,36]$. The main factors that affects the results of a direct georeferenced system can be grouped into four categories:

- Image sensor modeling

- Sensor placement

- Sensor synchronization

- Sensor errors

The first limiting factor is the image sensor modeling, which includes all criteria relating to the camera: the parameters of interior orientation, the camera geometry, the spatial resolution as well as the image capture rate [36]. The effect of the parameters of interior orientation on the georeferencing process has been addressed earlier in this chapter. The camera geometry and the spatial resolution are often grouped together as they are related. When selecting a camera for aerial surveys, the camera resolution plays an important role as higher resolutions provide better quality images which influences the precision of the final product. The camera geometry, which includes its shape and weight is an important factor to consider when conducting aerial surveys. When conducting aerial surveys using UAVs, there are often constraints for the maximum payload and location of a mounting surface, thus care should be taken when selecting a camera for the given application.

Secondly, the placement of the sensors on the aircraft can have a direct impact 
on the final results. Ideally, when choosing where to locate the sensors, there are two main criteria to be met; the first is to reduce the possibility of calibration errors on the lever arm measurements and the second is to eliminate any possible movement between sensors during flight $[4,36]$. To limit calibration errors on the lever arm measurements, having the sensors in the closest proximity as possible is the ideal configuration. However, the choice of location for sensors on aircrafts or UAVs are often limited; for example, on aircraft the GPS antenna is often located at the top of the fuselage and for the case of an aerial survey, the imaging sensor is facing downwards to capture ortho-photos and there is little to no room for adjustments [36]. A similar case can be made for the location of the IMU with respect to the GPS antenna and imaging sensor. Errors obtained when computing the lever arm between the IMU and GPS will have direct impact on positioning and thus will affect the results. In order to accurately determine the lever arm measurement between the IMU and GPS antenna, [4] proposes to use the GPS and IMU data obtained during a mission with a Kalman filter. However, when using smaller sized aircraft, such as small multirotor UAVs, it is possible to physically measure the lever arm using precise measuring tools, as was demonstrated in [31]. To limit the movement between the various onboard sensors, specifically between the IMU and imaging sensor, it is suggested that rigidly mounting the camera and IMU to a common surface can reduce errors.

Thirdly, one of the most important design criteria is synchronization, specifically the time synchronization between the IMU, GPS and image capture times. One method that is commonly employed to determine position and attitude information at a given time in order to determine exterior orientation is to compare the image capture times with logged GPS and IMU data. This process requires a high degree of accuracy due to the fact that errors in the synchronization times can significantly and 
directly affect the determination of the UAVs position and thus impact the results for the exterior orientation [36]. To reduce the possibility of synchronization errors it is recommended to calibrate the system using a highly accurate input/output timing board [4]. Lastly, it is important to consider the accuracy of each individual sensor which is being used in the system as their error will affect the final results [4].

\subsubsection{Integrated Sensor Orientation (ISO)}

Integrated sensor orientation, also called assisted aerial triangulation, is the third method of georeferencing as is discussed in [4,37-40]. ISO combines features from both direct and indirect georeferencing to estimate the exterior orientation parameters $[34,38,40]$. This method uses the exterior orientation parameters determined by direct georeferencing as initial estimates for the aerial triangulation process (indirect georeferencing) [38]. ISO can function with a limited number of ground control points which are employed to police errors in the final results. Kramer et al. [34] conducted two separate test cases using 4 and 21 ground control point and obtained virtually the same results and concluded that to use 4 to 6 ground control points was adequate to achieve correct and quality results [34].

The use of ISO presents many advantages such as eliminating the need for the boresight calibration and does not require an estimation for the orientation (roll, pitch and yaw) as these parameters can be determined during the aerial triangulation process $[34,39]$. Moreover, the computational time required to process the results is reduced due to the fully-automatic nature of the method [38]. However, one of the most promising advantages is this method's ability to obtain a comparable accuracy to higher precision direct georeferencing systems when using lower grade and more cost effect systems [38]. 


\subsubsection{Relevant Work}

This section presents a brief overview of the current research being done in the field of direct georeferencing for UAVs. In particular, this section aims to discuss the various systems being developed as well as the methods employed by the authors for the verification and validation of the data obtained through direct georeferencing.

Pfeifer et al. [8] studied direct georeferencing for lightweight UAVs using onboard components. A low-cost quadcopter, which had a maximum payload of $250 \mathrm{~g}$ and used open source software was employed for the study. Image acquisition was conducted using a compact digital camera. Through modifications to the source code of the UAV, the onboard sensors were synchronized with the digital camera and using the obtained raw data, the exterior orientation parameters were estimated [8]. The visible motion blur, which can appear on images, was reduced by using a very short exposure time $(1 / 1000 \mathrm{~s})$. Flight tests were conducted using 24 ground control points organized in a grid consisting of four rows of six points. The ground control points' positions were measured using a real-time kinematic (RTK) GPS having a $2 \mathrm{~cm}$ level of accuracy. The UAV flew along a predefined route through use of waypoints, stopping at each point for $10 \mathrm{~s}$. The exterior orientation values were estimated during post-processing by interpolating the exposure times. In order to verify and validate the results, the calculated values determined using direct georeferencing were compared to the results obtained through indirect georeferencing by means of bundle adjustment. Thus, the exterior orientation values obtained by indirect georeferencing could act as an absolute references.

Rehak et al. [31] developed a micro-UAV (MAV) with the capability of direct georeferencing. A custom vertical takeoff and land (VTOL) MAV, which utilized a multi-rotor design was developed as it allowed for all the necessary components 
needed to perform aerial surveys and photogrammetry to be mounted. Eight rotors were used to enhance the maximum payload $(1-1.5 \mathrm{~kg})$ and increase the level of redundancy in the event of an engine malfunction. The onboard software that was employed for the autopilot was Ardupilot which was developed by 3D Robotics. The exterior orientation values were obtained by relating the image acquisition time to the GPS logs. The authors delved further into the topic of image synchronization and studied the effect of camera lag on the acquisition time. They concluded that as long as the camera lag stayed constant, the value can be subtracted from the acquisition times and properly correlated to the GPS logs. Flight tests were carried out on an $8 \times 8 \mathrm{~m}$ calibration field where seven targets were dispersed and their positions measured using sub-cm accuracy using long-term GPS observations. A set of 46 images were captured. Then using a custom program, the exterior orientations were calculated. In order to verify and validate the results, the values obtained through direct georeferencing were compared to values obtained through indirect georeferencing by means of bundle adjustment. Thus, the exterior orientation values obtained by indirect georeferencing could act as an absolute references.

Turner et al. [5] studied direct georeferencing of ultrahigh-resolution UAV imagery using a multi-rotor UAV, having eight rotors and a maximum payload of $2 \mathrm{~kg}$. Image acquisition was done using a small format digital camera (Canon 550D DSLR, 15 megapixel). Furthermore, a single-board computer was used to log the GPS data during flight and the camera position where obtained by synchronizing the GPS logs with the image acquisition times. Once the camera positions were determined from the logs, the values were corrected to take into consideration the distance between the GPS antenna location and the camera center, which was fixed. Moreover, the authors determined the camera lag in order to study if it had any significant effect on the results. Using the maximum allowable shutter speed and multiplying it by 
the speed of flight, the authors determined the worst-case scenario discrepancy of the camera positions due to movement. Comparing this value with that of the accuracy of the GPS system being employed, the positional error due to the synchronizations delays can be determined to gage if its negligible. Flight tests were conducted on a 0.8-ha section of farm land using 22 ground control points whose positions were measured using survey grade differential GPS (DGPS) with typical accuracy of $2 \mathrm{~cm}$ in the horizontal and $4 \mathrm{~cm}$ in the vertical directions respectively. In order to verify and validate the results, the values obtained through direct georeferencing were compared to values obtained through indirect georeferencing by means of bundle adjustment.

Finally, the authors conducted tests using three different image processing softwares; Photoscan, Pix4d and a custom bundler technique. Photoscan proved to be the best option in terms of both processing time and mean absolute total error [5]. It is for this reason that Photoscan was chosen as the software to process the results of this thesis.

The research being conducted by $[5,8,31]$ demonstrates the accepted means for verification and validation of direct georeferencing systems. Experimental results are compared to values determined through use of indirect georeferencing, obtained by using multiple ground control points whose positions have been measured using highprecision equipment. Though each author is attempting to address the same issue, the unique aspect of their research lies in the various types of UAVs, sensors and data acquisition systems that are being employed. Another interesting feature of current research is the use of open-source components such as the Ardupilot software. The former is one of the factors which influenced the work presented in this thesis. 


\section{Chapter 3}

\section{Experimental Methodology}

\subsection{System Overview}

The literature review presented in Chapter 2 identified certain gaps in technology regarding UAV systems for aerial mapping/surveying. Firstly, it was concluded that there is a benefit to researching direct georeferencing and integrated sensor orientation systems as they can greatly reduce the time and cost of a survey when compared to the traditional indirect methods of georeferencing. Secondly, the main limiting factors which affect direct georeferencing systems were identified as being: image sensor modeling, sensor placement, sensor errors and synchronization. The research presented in this thesis aims to provide a low-cost solution to improve the current method of direct georeferencing and integrated sensor orientation systems for close range photogrammetry, through added onboard computational functionality. To this end, a Raspberry Pi 3 B single board computer was connected to the autopilot of a 3DR Iris+ UAV. Installing Dronekit-python development tool onto the Raspberry Pi enabled direct access to the various aircraft parameters, most notably its position, altitude and attitude, which are all essential to the direct georeferencing and 
integrated sensor orientation process. Furthermore, by integrating the Raspberry Pi camera V2, which can be controlled and programmed in Python, it was possible to directly associate the position (latitude, longitude and altitude) and orientation (roll, pitch and yaw) with the corresponding image during flight. The former eliminates the need to synchronize the image capture times with GPS and telemetry logs, which is the method employed in current direct georeferencing systems.

In this section of the thesis, the aircraft's hardware as well as the software used for this research is presented and explained. Lastly, the experimental methodology and the conducted tests are discussed. All results are presented in Chapter 4.

\subsubsection{Aircraft Hardware}

The system that was implemented for the research is shown in Figure 3.1.

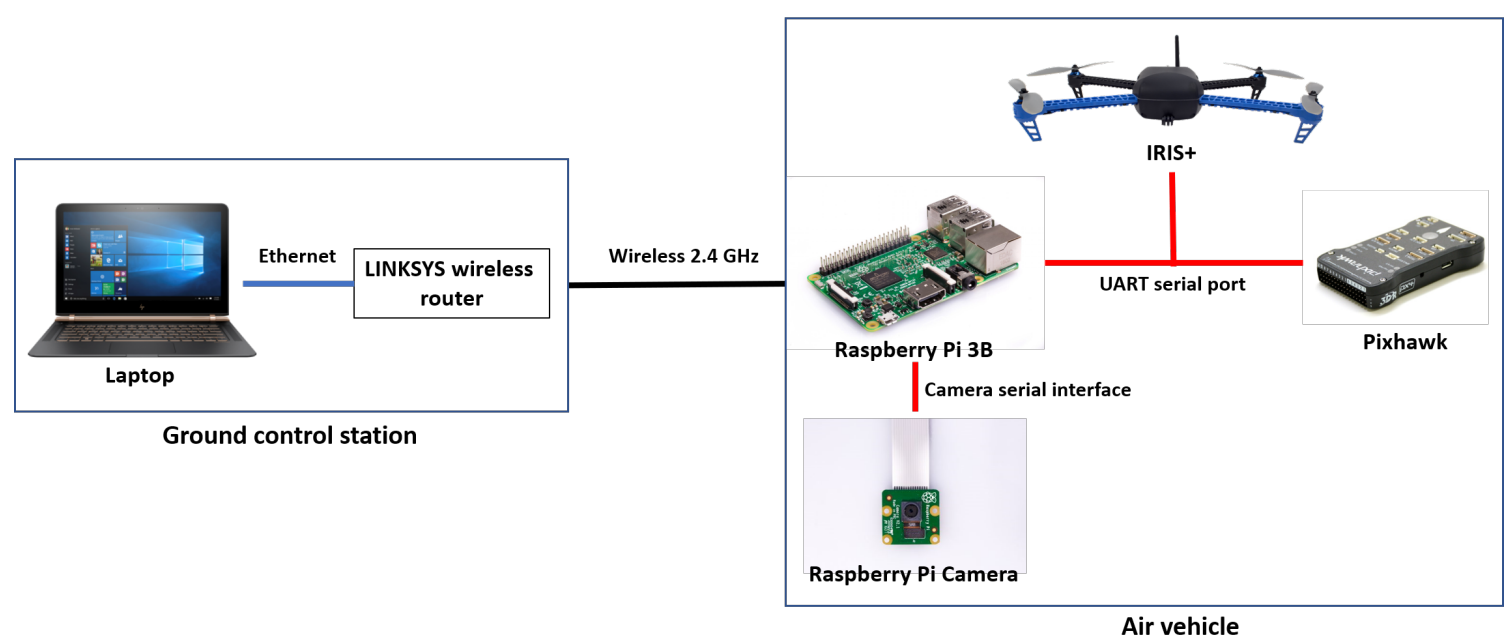

Figure 3.1: System overview, modified from [41-43]

A Raspberry Pi single board computer was connected to the onboard Pixhawk PX4 autopilot via the UART serial port. The Raspberry Pi camera V2 was connected to 
the single board computer using the camera serial interface. These two components were mounted onto the bottom of the UAV and are shown in Figure 3.2.

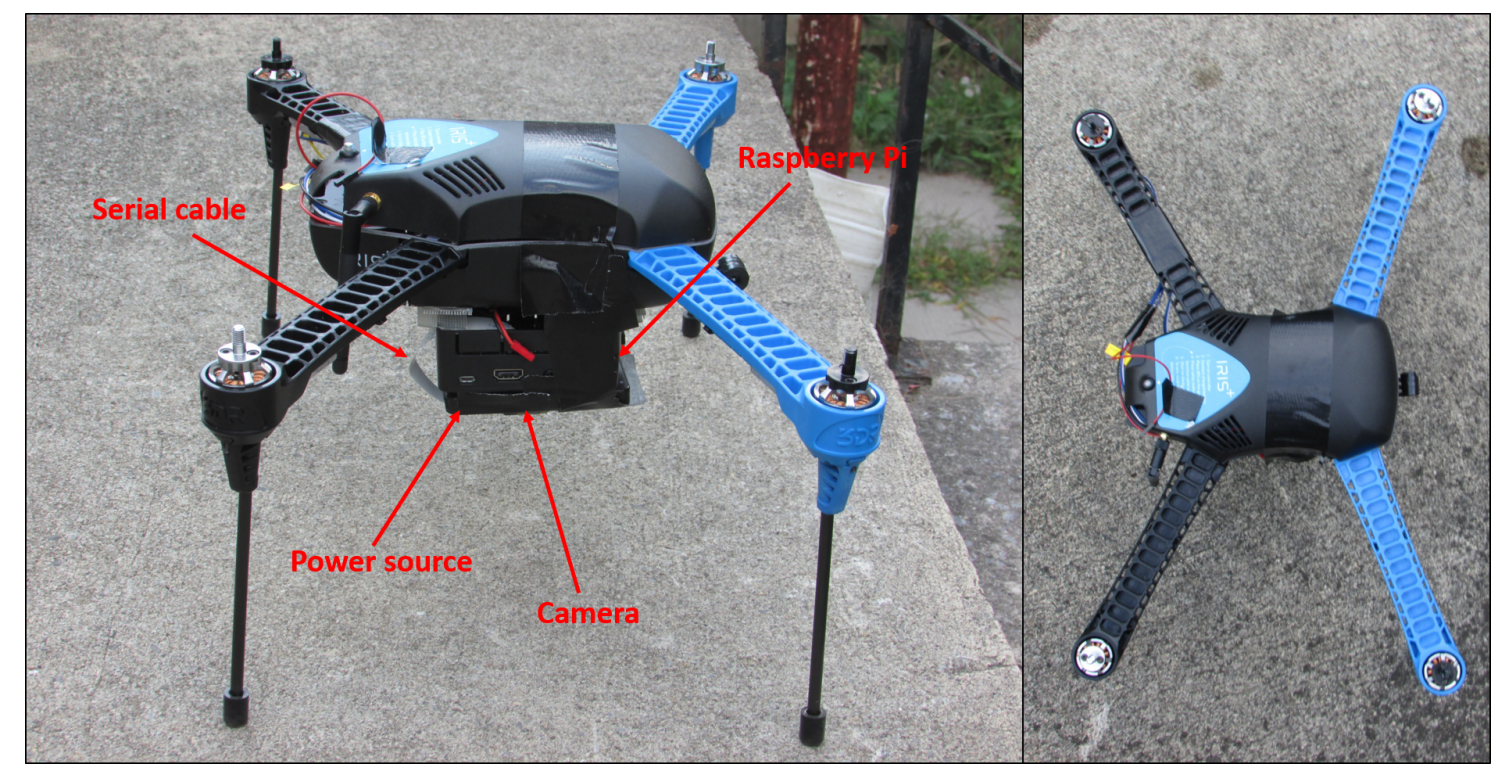

Figure 3.2: Components mounted on the Iris+

A laptop running Mission Planner was used as a ground control station during testing. Using the telemetry adapter in tandem with Mission Planner allowed for the UAV to be controlled from the laptop and for the telemetry logs to be directly saved as well. Lastly, using a wireless connection enabled remote access via a secure shell (SSH) to the Raspberry Pi during flight to both start and end the python script used to capture and geotag the images.

\subsubsection{3D Robotics Iris+ Uninhabited Aerial Vehicle}

The Iris + is a $\mathrm{x}$-frame quadcopter developed by 3D Robotics for commercial applications. The UAV has a maximum payload capacity of $425 \mathrm{~g}$, a radio range of $1 \mathrm{~km}$ and an estimated maximum flight of 16-22 minutes depending on factors such as weather and the size of the payload. The aircraft employs the Pixhawk PX4 as its autopilot 
hardware with the APM:Copter 3.1 firmware, installed through the use of Mission Planner and is powered by a 3 cell $11.1 \mathrm{~V} 3.5$ Ah lithium polymer battery. The Iris+ employs a 3DR uBlox GPS with compass LEA-6H module $5 \mathrm{~Hz}$ for positioning and either a 915 (North America) or 433 (Europe and Asia) mHz telemetry radio V2.

\subsubsection{Pixhawk PX4 Autopilot}

The Pixhawk PX4, shown in Figure 3.3, is an autopilot system which can be used with fixed wing, multi-rotor or any other moving robotic platforms. It houses three processors; a 32-bit ARM Cortex M4 core with floating-point-unit (FPU), a 168 $\mathrm{MHz} / 256 \mathrm{~KB} \mathrm{RAM} / 2 \mathrm{MB}$ flash and a 32-bit failsafe co-processor. Moreover, the Pixhawk contains a multitude of onboard sensors such as:

- ST Micro L3GD20H 3-axis 16-bit gyroscope

- ST Micro LSM303D 3-axis 14-bit accelerometer/magnetometer

- MEAS MS5611 barometer

- MPU 6000 3-axis accelerometer/magnetometer

Figure 3.3 also shows the available interfaces. For this research the Raspberry Pi 3 B single board computer was connected to the Pixhawk through Telem 2, which is a UART serial port. Table 3.1 lists the mechanical properties of the Pixhawk autopilot. 

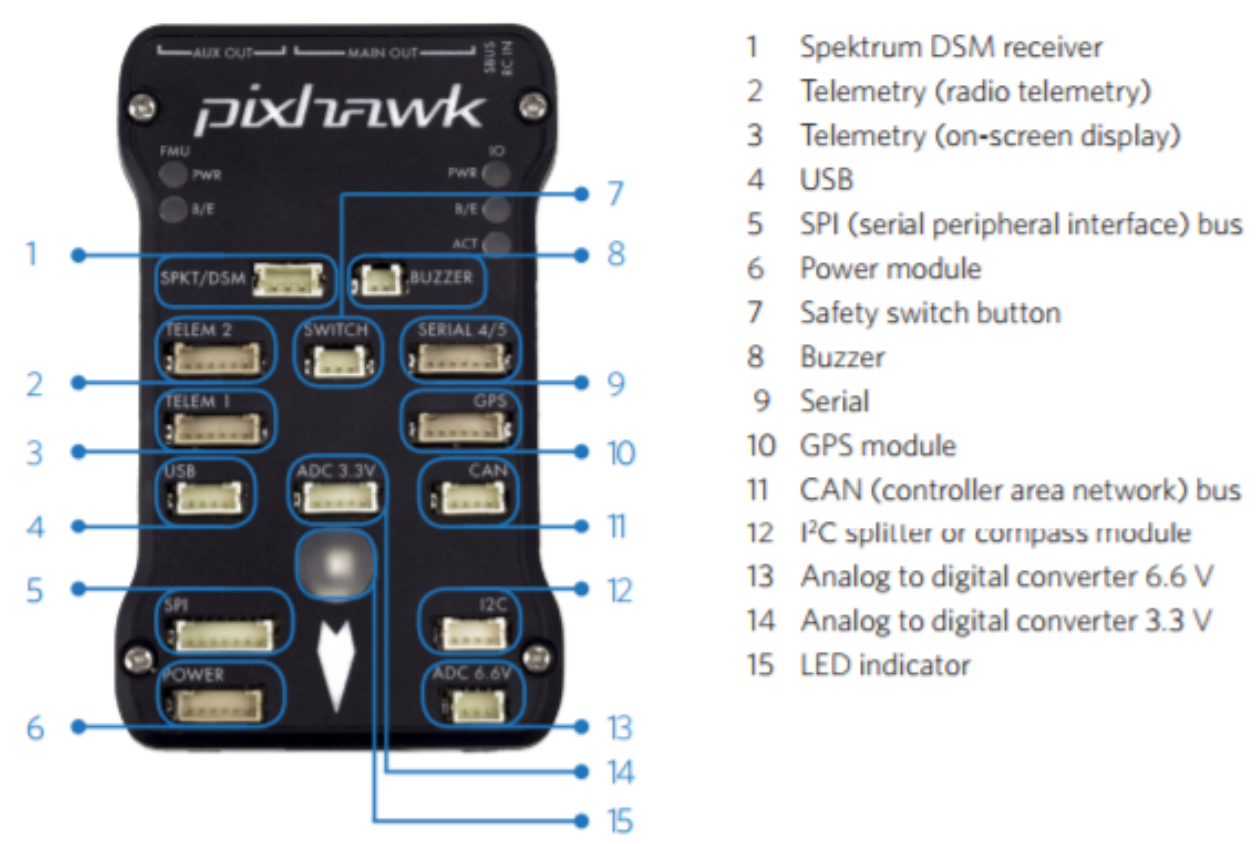

Figure 3.3: Pixhawk PX4 [44]

Table 3.1: Pixhawk mechanical properties [44]

\begin{tabular}{|c|c|}
\hline Weight & $38 \mathrm{~g}$ \\
\hline Width & $50 \mathrm{~mm}$ \\
\hline Height & $15.5 \mathrm{~mm}$ \\
\hline Length & $81.5 \mathrm{~mm}$ \\
\hline
\end{tabular}

\subsubsection{Raspberry Pi 3B Single Board Computer}

The Raspberry Pi 3B, shown in Figure 3.4, is the third-generation model of the Raspberry pi single board computer that has built in wireless LAN and Bluetooth 4.1 connectivity. The device has $1 \mathrm{~GB}$ of RAM and employs a quad core $1.2 \mathrm{GHz}$ Broadcom BCM2837 chip with a 64 bit processor. Furthermore, there are 40 general purpose input/output (GPIO) pins that can be used for integration with a variety of sensors such as: temperature, infrared, light detection and ranging (LIDAR), ultrasonic and as for the case with the research presented in this thesis, imaging sensors. 
The board also houses four universal serial bus 2 (USB 2) ports, a full size highdefinition multimedia interface (HDMI) port, a camera serial interface (CSI) camera port which is used in order to connect the Raspberry Pi camera V2. Moreover, there is a display serial interface (DSI) port which allows for a touch screen display to be connected to the device. The device functions using a Linux-based operating system called Raspbian Jessie version 2017-07-05 that was installed onto a micro SD card.

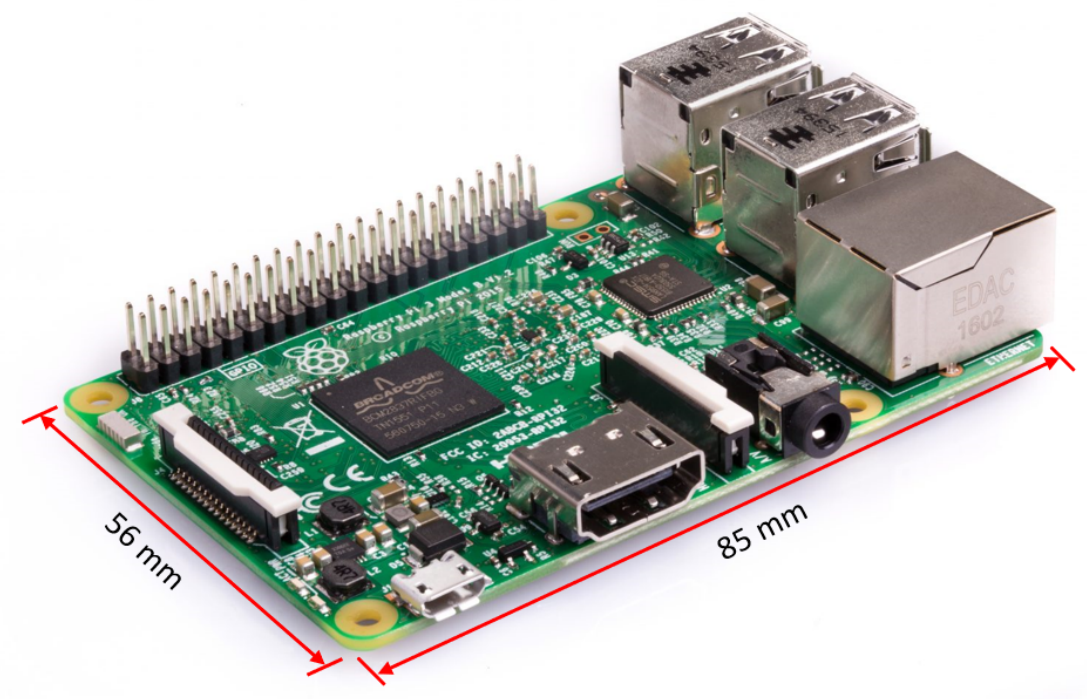

Figure 3.4: Raspberry Pi model 3B [42]

\subsubsection{Raspberry Pi Camera V2}

The Raspberry Pi camera module V2, shown in Figure 3.5, is a small and compact digital camera that employs an 8-megapixel Sony IMX219 imaging sensor capable of capturing high-definition video as well as images. Furthermore, the sensor has a resolution of $3280 \times 2464$ pixels and an image area of $3.68 \mathrm{~mm} \times 2.76 \mathrm{~mm}$ with the pixel size being $1.12 \mu \mathrm{m} \times 1.12 \mu \mathrm{m}$. Piras et al. [45] conducted research into the application of the Raspberry Pi camera V2 for capturing images for photogrammetry 
applications. Their work noted the many advantages of the camera, with the former primarily being its low cost (under $35 \mathrm{CAD})$, light weight (3 g) and the simplicity involved with connecting and controlling the camera through the use of the Raspberry $\mathrm{Pi}$ [45]. The camera is connected to the device via the camera serial interface and can be controlled using the functions available in the Picamera python library.

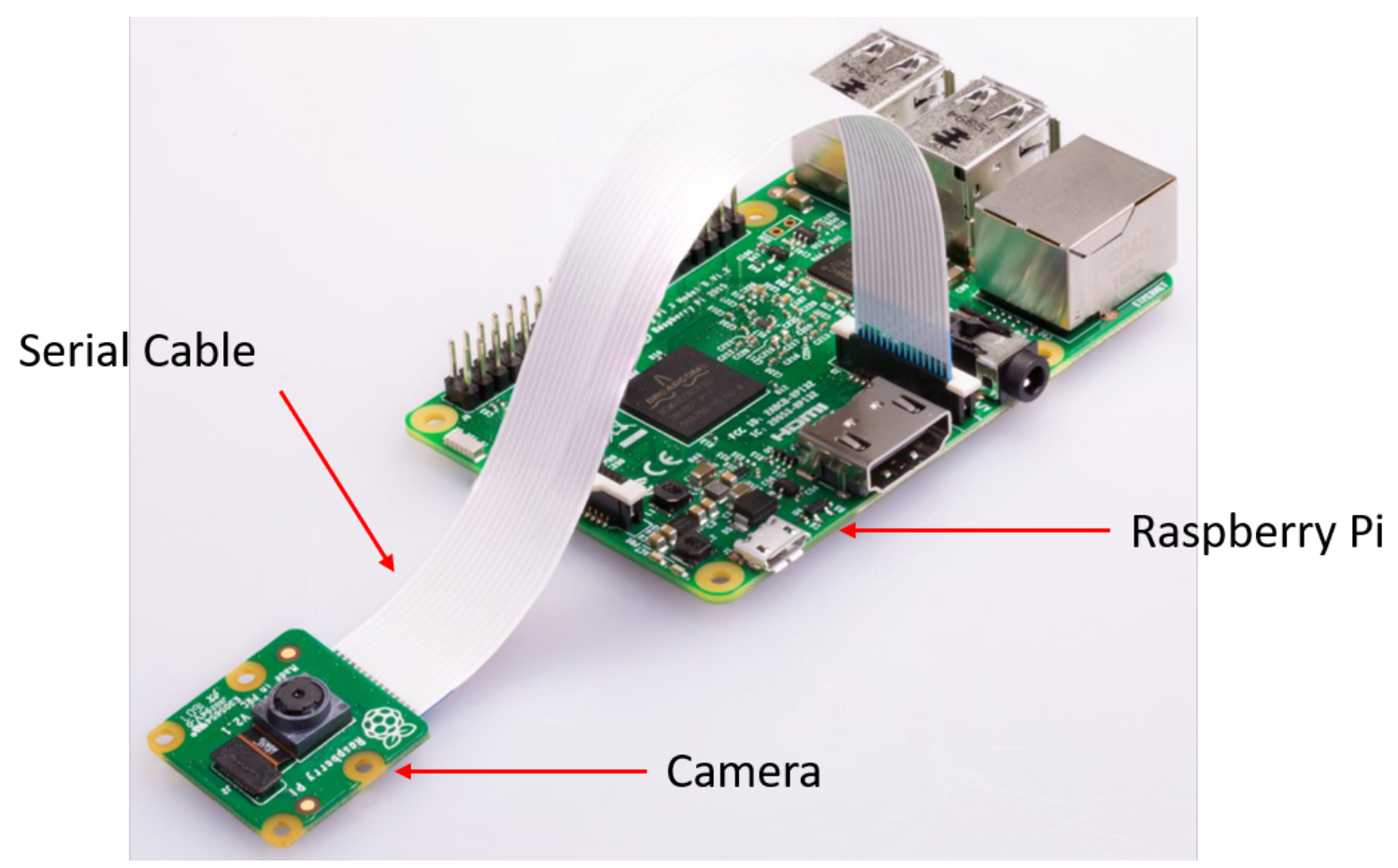

Figure 3.5: Raspberry Pi camera V2 [41]

The Pi camera documentation provides the focal length of the camera, which is $3.04 \mathrm{~mm}$. However, it does not provide the coordinates of the principal point. The interior orientation parameters of the Raspberry Pi camera V2 were verified in [45] and it was determined that the focal length and coordinates of the principal point were $1.14 \mathrm{~mm}, 0.507 \mathrm{~mm}$ and $0.395 \mathrm{~mm}$ respectively. These parameters will be used for further calculations presented in this thesis. Table 3.2 summarizes the mechanical characteristics of the Pi camera. 
Table 3.2: Raspberry Pi Camera V2 physical characteristics

\begin{tabular}{|c|c|}
\hline Length & $23.86 \mathrm{~mm}$ \\
\hline Width & $25 \mathrm{~mm}$ \\
\hline Height & $9 \mathrm{~mm}$ \\
\hline Horizontal FOV & $62.2 \mathrm{deg}$ \\
\hline Vertical FOV & $48.8 \mathrm{deg}$ \\
\hline
\end{tabular}

\subsubsection{Software}

The primary software used were Mission Planner, Dronekit and Agisoft's PhotoScan. Flight planning and the configuration of the Iris+ were done using Mission Planner while Dronekit was installed in this research on the Raspberry Pi 3B single board computer and was used to create a script for image and data acquisition. Lastly, Agisoft's PhotoScan was used to process the images and generate point clouds for comparisons. In this section of the thesis, the various software that were integrated and used during the research will be presented and explained.

\subsubsection{Mission Planner}

Mission Planner is a free open source ground control station software for the Pixhawk, Plane and Rover firmware that was developed by ArduPilot for Windows, with the application being for systems employing the open source Ardupilot autopilot [46]. This software allows the users to install the firmware into the autopilot and to configure as well as customize the vehicle's setting in order to meet the desired performance. Furthermore, it allows the creation of autonomous missions in a graphical user interface (GUI) which can be saved and loaded into the autopilot at a later date [46]. Moreover, given the proper telemetry equipment the following features can be achieved [46]: 
- Monitoring the vehicle's parameters during flight.

- Operating the vehicle in first person view (FPV).

- Enabling the recording and analysis of telemetry log files.

Figure 3.6 illustrates Mission Planner's GUI and describes the various parameters that can be seen during a mission. It is important to note that all values for the parameters that are shown in Figure 3.6 will be recorded to a log file regardless of whether the autopilot is connected to a device (either a laptop, tablet or phone).

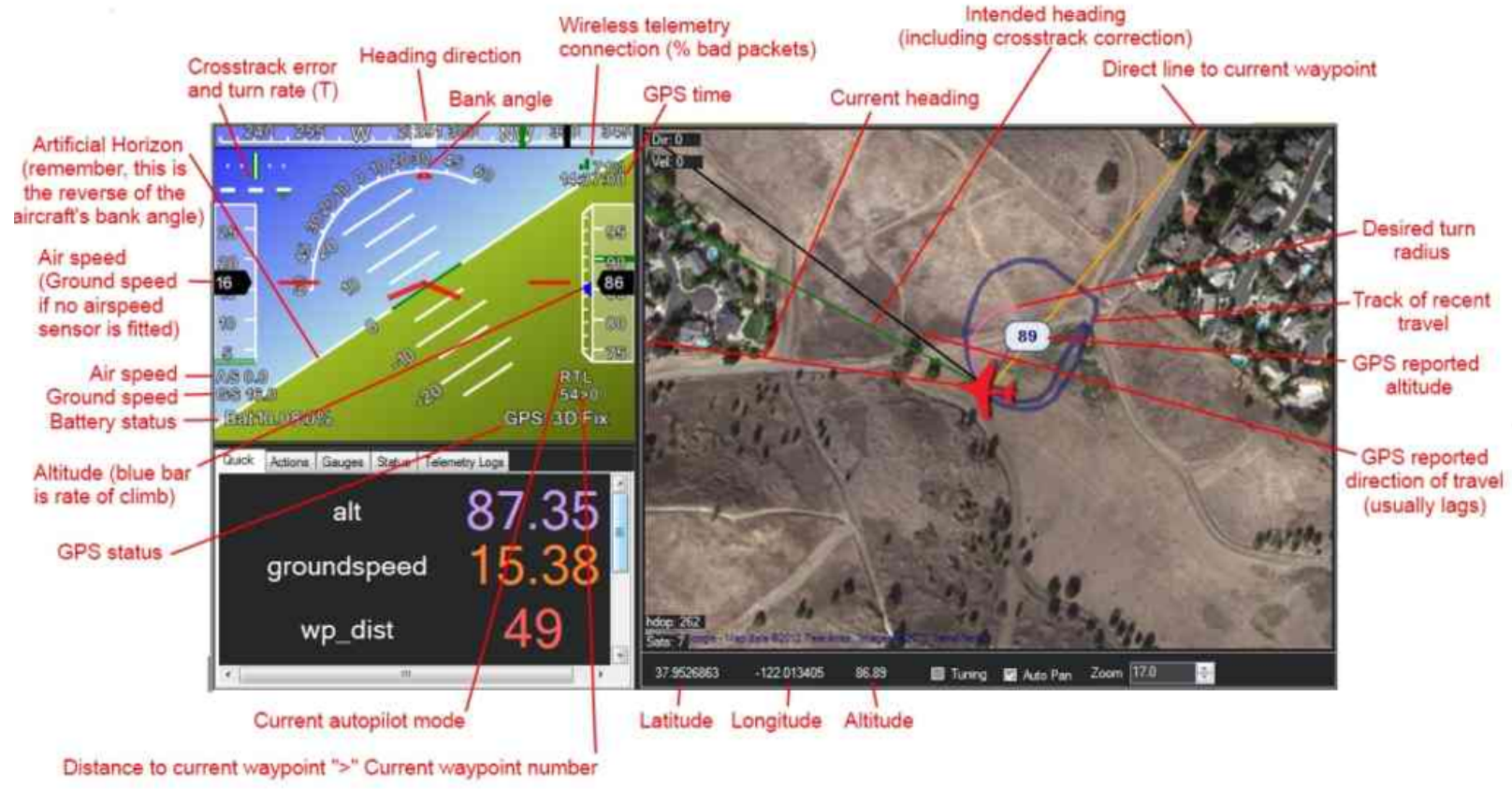

Figure 3.6: Mission Planner GUI [46]

One of Mission Planner's most relevant features is the flight plan. Mission Planner allows the user to create missions that will be flown autonomously by waypoint navigation. The user defines a set of intermittent position objectives to which the UAV will fly to once the autopilot's mode has been switched to AUTO. Furthermore, during flight planning it is possible to use MAVLink mission commands which allow 
the user to create complex navigation programs beyond simple travel between waypoints. Figure 3.7 demonstrates the flight planning section and shows the complete list of available MAVLink mission commands.

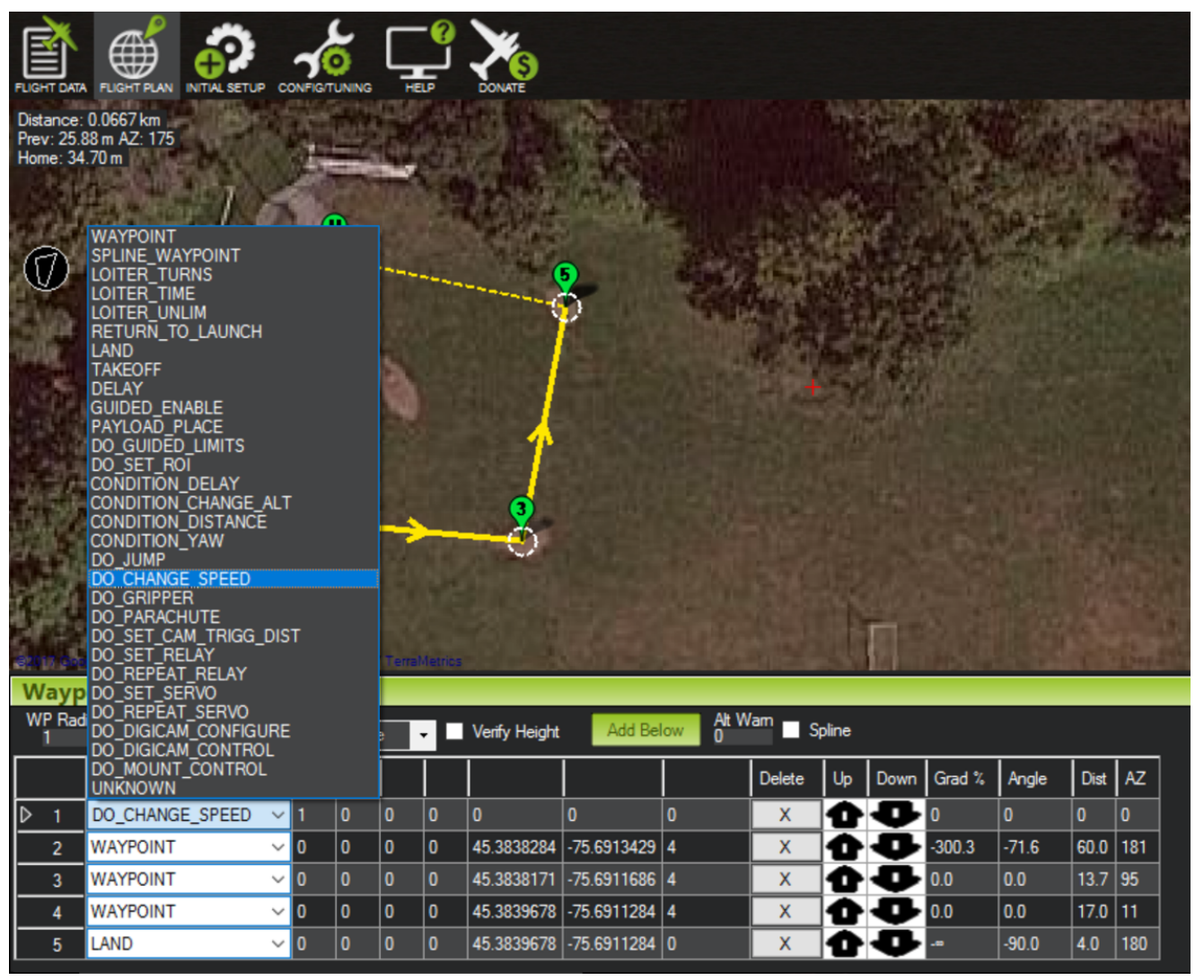

Figure 3.7: Mission Planner MAVLink mission command list

The commands are grouped into three categories; navigation, DO and conditional. The navigation category includes all commands which affect the motion of the vehicle while the conditional commands are used to control the execution of the DO commands [46]. These commands are executed sequentially and there can only be one navigation running in tangent to a single DO or conditional command at a given time [46]. Mission Planner's simplicity and ability to create complex flight plans using the mission commands were some of the main reasons that it was chosen for this research. The main commands that were used during flight plaining for the 
experiments described in the next section were:

- TAKEOFF

- DO CHANGE SPEED

- DELAY

- WAYPOINT

Each of the items listed above will be addressed in greater detail in Section 3.2.

\subsubsection{Dronekit}

Dronekit is an open source application development tool which was developed by 3D Robotics which runs on Linux, MAC as well as Windows operating sytem and is compatible with all of 3DR's UAVs [47]. There are three versions of Dronekit; Python, Android and Cloud. The Python version allows the user to create applications that run on companion computers such as the Raspberry Pi. The former can enable programs that are computationally-intensive such as optical flow, 3D modeling and path planning to be executed at the same time as the autopilot is running other functions and missions [47]. Dronekit provides a detailed application program interface (API) which enables communications to a vehicle using MAVLink. This gives the users access to the vehicle's telemetry, state and parameter variables, which was an important feature used for this research [47]. Furthermore, it's possible to control and dictate the motion of a vehicle simply using a python script. The aforementioned features allow for the development of more complex and intelligent autonomous UAVs. Similarly, the android version of Dronekit, allows for the creation of applications intended 
for android devices while the cloud version enables the development of web services that communicate directly with a UAV [47].

As mentioned in Section 3.1.1, the Raspberry Pi camera V2 was chosen as the image capturing device for this research. The camera can be programmed through the use of various functions available in a python library. Seeing how the camera functions using python, Dronekit-Python became the obvious choice as a means to program the data acquisition system employed on the Iris + as both the UAV and camera could be controlled using a single script.

\subsubsection{Agisoft PhotoScan}

The software that was used to process the captured images for analysis was Agisoft's PhotoScan Professional Edition version 1.2.6. It is an advanced image-based 3D modeling tool which can produce 3D models given multiple sets of overlapping images [48]. Furthermore, it also has built-in features for georeferencing said models.

This software provides users with the option of importing the exterior orientation parameters of the captured images in either the universal transverse mercator (UTM) or world geodetic system (WGS84) coordinate systems. This information can greatly reduce the aerial triangulation process (see Section 2.3.3) and will produce a georeferenced 3D model once the image processing is completed. Should the exterior orientation parameters not be available, the produced models will simply be in a local frame of reference. Moreover, when the exterior orientation parameters are not given, PhotoScan is able to calculate them using the principles of indirect georeferencing (see Section 2.3.2).

A model can be georeferenced if either the exterior orientation parameters of 
each image is known (i.e directly georeferenced) or if there are certain markers in the images whose coordinates have been determined using some type of positioning system (i.e indirectly georeferenced using ground control points). One of PhotoScan's most relevant features used for this research is the software's ability to determine camera position and orientation, and to export these values to a textfile. Once the captured images have been processed, the camera positions can be exported by selecting export as "omega phi kappa" or "xml". Using the "omega phi kappa" tool produces a textfile including the name of the image as well as:

- 3 rows with camera position: $X, Y, Z$ i.e the position of the camera's center.

- 3 rows with attitude angles of the camera (i.e roll, pitch and yaw).

- 9 elements of a rotation matrix determined from the attitude angles of the camera.

It is important to note that if the project is georeferenced, the values for $X Y Z$ will have the following form:

- Latitude [deg] Longitude [deg] Height [m] of the camera's perspective center.

The "xml" option provides the users with a series of 16 numbers under the label of transform, associated to each captured image. These 16 numbers correspond to a 4 $\mathrm{x} 4$ rotation matrix as well as a $4 \mathrm{x} 1$ translational component in homogeneous coordinates. Additional mathematical operations are required to determine the camera's exterior orientation parameters from the given data. The former is the reason that for this research, results were exported using the "omega phi kappa" format. PhotoScan was chosen as the processing software for this research primarily due to its availability. Moreover, as stated in Section 2.3.5, Turner et al. [5] compared PhotoScan with 
two other image-based 3D modeling software packages; Pix4D and a custom Bundler technique and concluded that PhotoScan required $60 \%$ less of the total processing time, while obtaining a mean absolute error of $0.1149 \mathrm{~m}$. This was an improvement of $0.1322 \mathrm{~m}$ compared to the closest viable alternative.

\section{$3.2 \quad$ Testing}

\subsubsection{Preliminary Testing}

\section{Connecting Raspberry Pi to Pixhawk}

In order to enable two-way communication between the Raspberry Pi and the Pixhawk autopilot, the serial port TELEM 2 on the Pixhawk was connected to the transmission $(\mathrm{Tx})$, receive $(\mathrm{Rx})$ and ground input/output pins on the Raspberry Pi. Figure 3.8 illustrates the wiring setup used for this research.

A 6 pin DF13 cable was employed to connect to the TELEM 2 serial port while its ends were spliced and soldered to a series of four breadboard jumper wires, each having a female end which connected to the Raspberry Pi's GPIO pins mentioned above. It is important to note that the transmission $(\mathrm{Tx})$ and receive $(\mathrm{Rx})$ pins on the TELEM 2 port should be connected to the Rx and Tx pins on the Raspberry Pi, respectively. The Pixhawk's TELEM 2 port includes a $5 \mathrm{~V}$ pin which can be used to power the Raspberry $\mathrm{Pi}$; however, it is unregulated and during initial trials it was consistently observed that the Pixhawk did not continuously provide $5 \mathrm{~V}$, which caused the Raspberry Pi to shutdown during operation. Due to this issue, an external $5 \mathrm{~V}$ power source was chosen to power the device via micro-USB, which is regulated on the Raspberry Pi. Lastly, prior to establishing communication between both devices, 


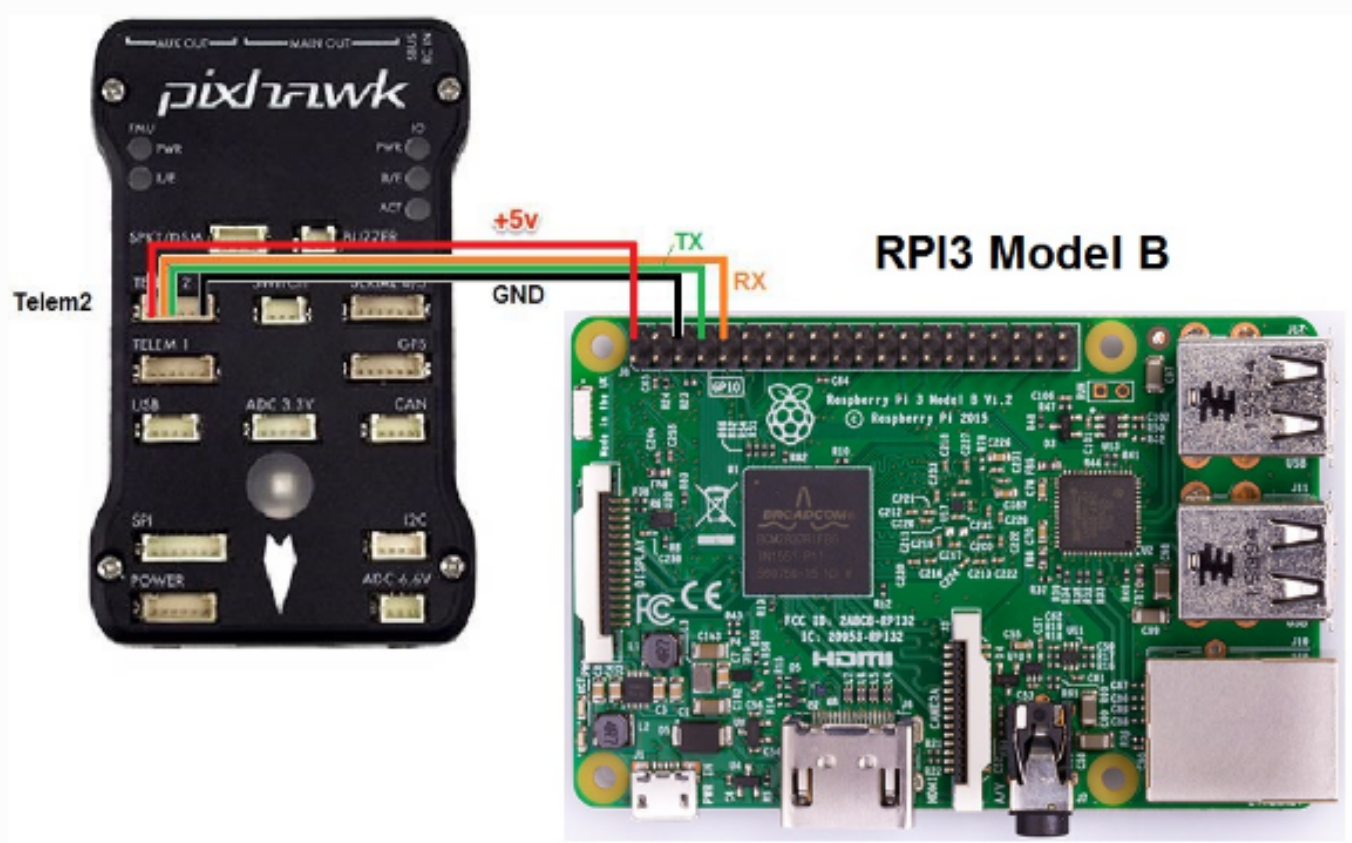

Figure 3.8: Connection between Raspberry Pi and Pixhawk autopilot [49]

there are certain libraries that should be downloaded onto the Raspberry Pi. These items as well as the steps needed to install them on Linux are detailed in the Ardupilot documentation [49].

\section{Data Acquisition with Dronekit}

Through use of the Dronekit-Python libraries, which enabled programming access to the aircraft's parameters, a custom data acquisition script was written in order to directly associate the relevant telemetry information to the respective image being captured in real time. A flowchart which details the functions of the data acquisition script is shown in Figure 3.9.

The first step in the script is to connect to the vehicle. If a connection cannot be established, an error is produced and the script does not run. Once a connection is established, a new folder is created in a predetermined location on the Raspberry Pi which has the following format, time-month-day-year. Next, the Raspberry Pi 


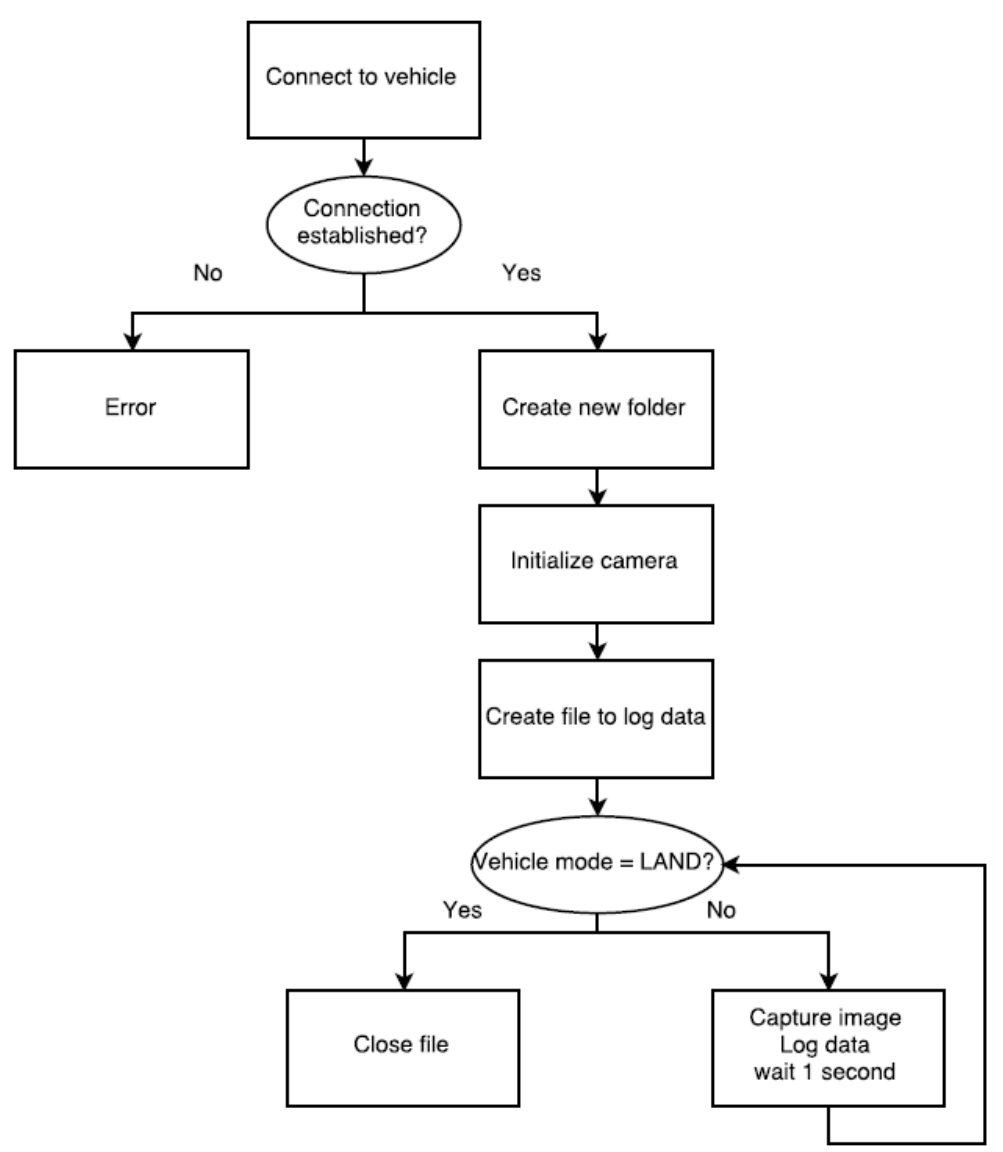

Figure 3.9: Data acquisition script flowchart

camera is initialized and a file is created to log the relevant information and is made writable. Once these preliminary steps are completed, the main loop begins. During the mission, the vehicle's mode is set to AUTO. During this time an image is captured at a given interval set by the user. For the tests conducted in this research, the time between images was set to 1 second. Whenever an image is captured, the following information is written to the log file: image name, position (latitude, longitude and altitude ASL) and attitude (roll, pitch and yaw) of the aircraft. Once the aircraft completes its final waypoint, the vehicle mode changes to LAND, at this point the data acquisition script stops recording data and closes the log file. The data can later be retrieved on the Raspberry Pi. 
There were two methods which were explored to activate the data acquisition script while at the flight field; manually starting the script by remotely logging into the Raspberry Pi using a secure shell (SSH) and having the script run automatically on start up of the Raspberry Pi. The first method, using another device to SSH into the Raspberry Pi required knowledge of the devices IP address as well as a wireless connection. Then using PuTTY, which is an SSH client installed on an accompanying laptop running Windows, the script could be started prior to flight.

The second method, having the script run automatically on start up is slightly more involved as it requires the user to run a shell script to schedule an event which will run on start up. This event would contain a few lines of code which provide the necessary commands to start the data acquisition script. However, using this method presents certain disadvantages such as the script may only be started once, if the user wanted to fly a second time, the Raspberry Pi would have to be restarted. Moreover, since the user cannot see or monitor the code during flight, the only way to confirm that the code functioned properly would be after the flight, which is not convenient. Furthermore, during preliminary trials this method was found to be unreliable. Based on this information, the first method was chosen as it was both practical and reliable. Since Carleton's River Field does not have access to a wireless signal, which is required to SSH into the Raspberry Pi, a wireless router was brought to the field and powered by a portable battery. All the information during flight was monitored at the ground control station shown in Figure 3.10. 


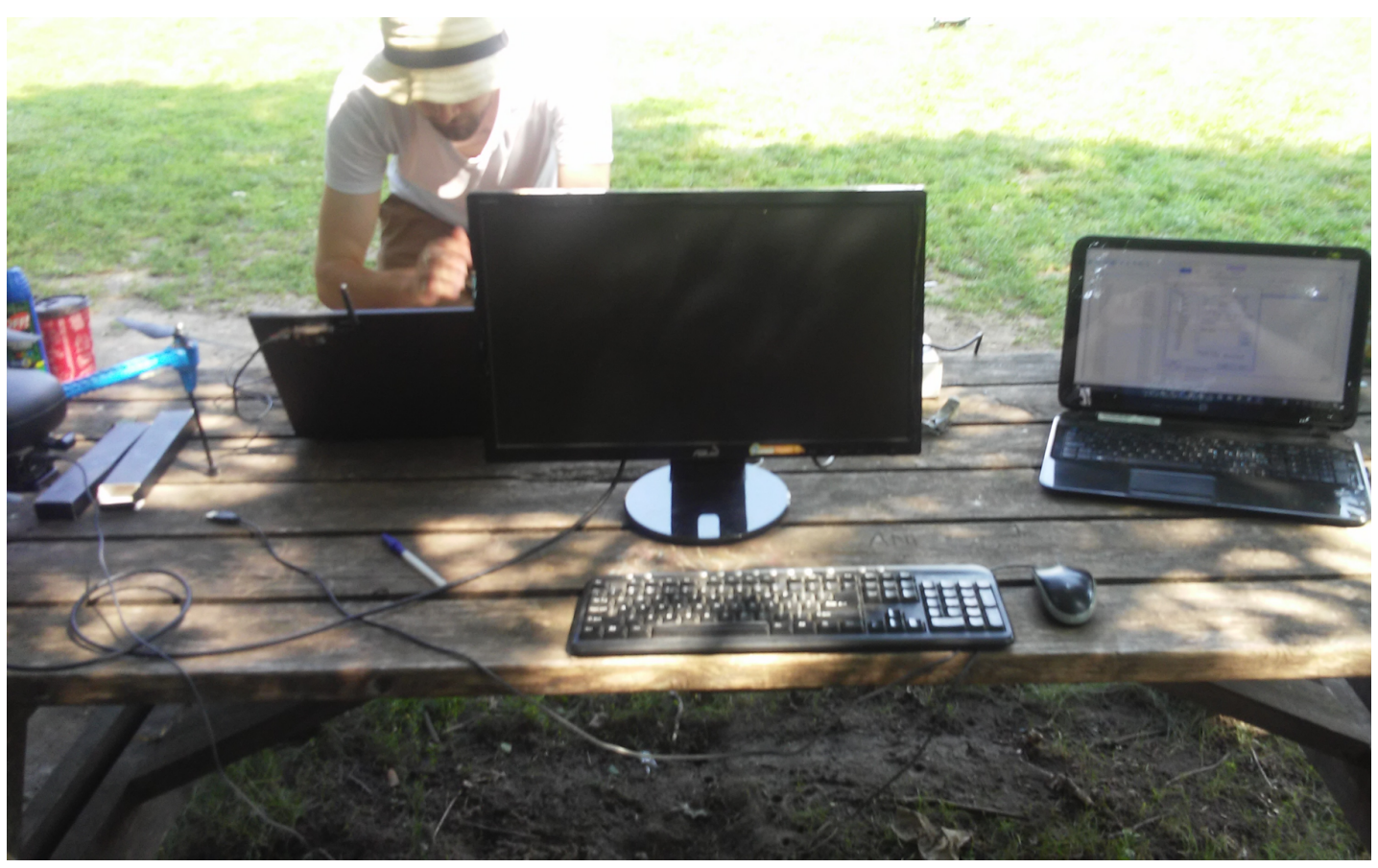

Figure 3.10: Ground control station set-up at the Carleton River Field test site

In the image, the laptop was used to remotely connect to the Raspberry Pi, start the script as well as to monitor the code while it was executing. The monitor and keyboard were primarily used for troubleshooting.

\subsubsection{River Field Case Study}

An aerial survey was carried out on Carleton's River Field in order to verify the functionality of the data acquisition script implemented on the Iris + during flight. This site was chosen primarily due to convenience and its proximity to the University compared to alternative test sites. A series of three test flights were conducted to obtain aerial images which were used to produce 3D point clouds using the three methods of georeferencing. This section details all the pre-flight and in-flight steps that were followed to acquire and process the data. Figure 3.11 shows a satellite 
image of the River Field baseball diamond which was surveyed.

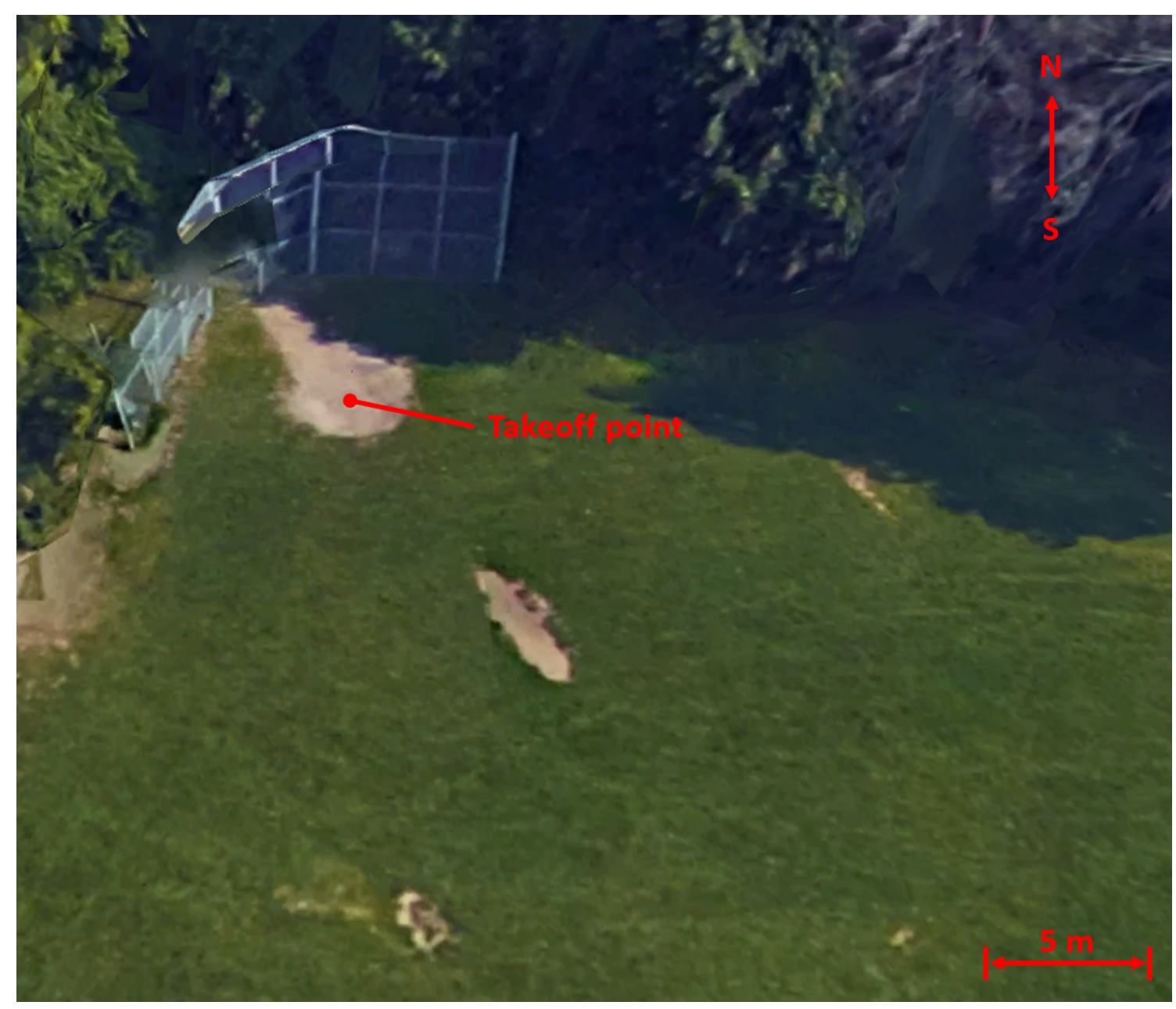

Figure 3.11: River Field Google satellite image

\subsubsection{Special Flight Operations Certificate}

A special flight operation certificate (SFOC) provides permission from Transport Canada to operate a UAV for non-recreational use such as for academic research, surveying as well as aerial photography and videography [50]. The certificate dictates the manner and location where the applicant(s) may fly. In order to test the added features implemented on the Iris+, a case study was conducted on the baseball diamond of River Field, located on the campus of Carleton University. Due to nature of 
the flights (for research purposes) and the proximity of the test site to neighboring structures as well as the possibility of encountering spectators, an SFOC was required. As per the terms of the SFOC obtained, all flight operations were conducted under the following conditions:

- Daytime Visual Flight Rules (VFR)

- Light wind (10 kts max)

- Minimum visibility of $2 \mathrm{Nm}$

- Minimum ceiling of $1000 \mathrm{ft}$. AGL or higher

- No precipitation

- Visual line of sight (VLOS)

- Maximum altitude $100 \mathrm{ft}$ above ground level (AGL)

To alleviate some of the concerns for the presence of casual spectators, prior to each day of flying, the Carleton University Departments of University Safety and Risk and Insurance were contacted in an effort to ensure that any potential spectator remained at a safe distance, $100 \mathrm{ft}$ laterally away from the UAV during operations. Moreover, the Department of University Athletics was also contacted to verify the availability of the field at the desired time as well as to ensure that there were no scheduling conflicts.

\subsubsection{Preflight work}

Prior to conducting the aerial survey of River field, some preparatory work was required. The former included choosing and placing the ground control points as well 
as measuring their positions. For the series of flights outlined in this section, five control points were placed on the first, second and third bases as well as the pitchers mount and home plate. Figure 3.12 shows a sample of the targeted control point employed throughout the survey.

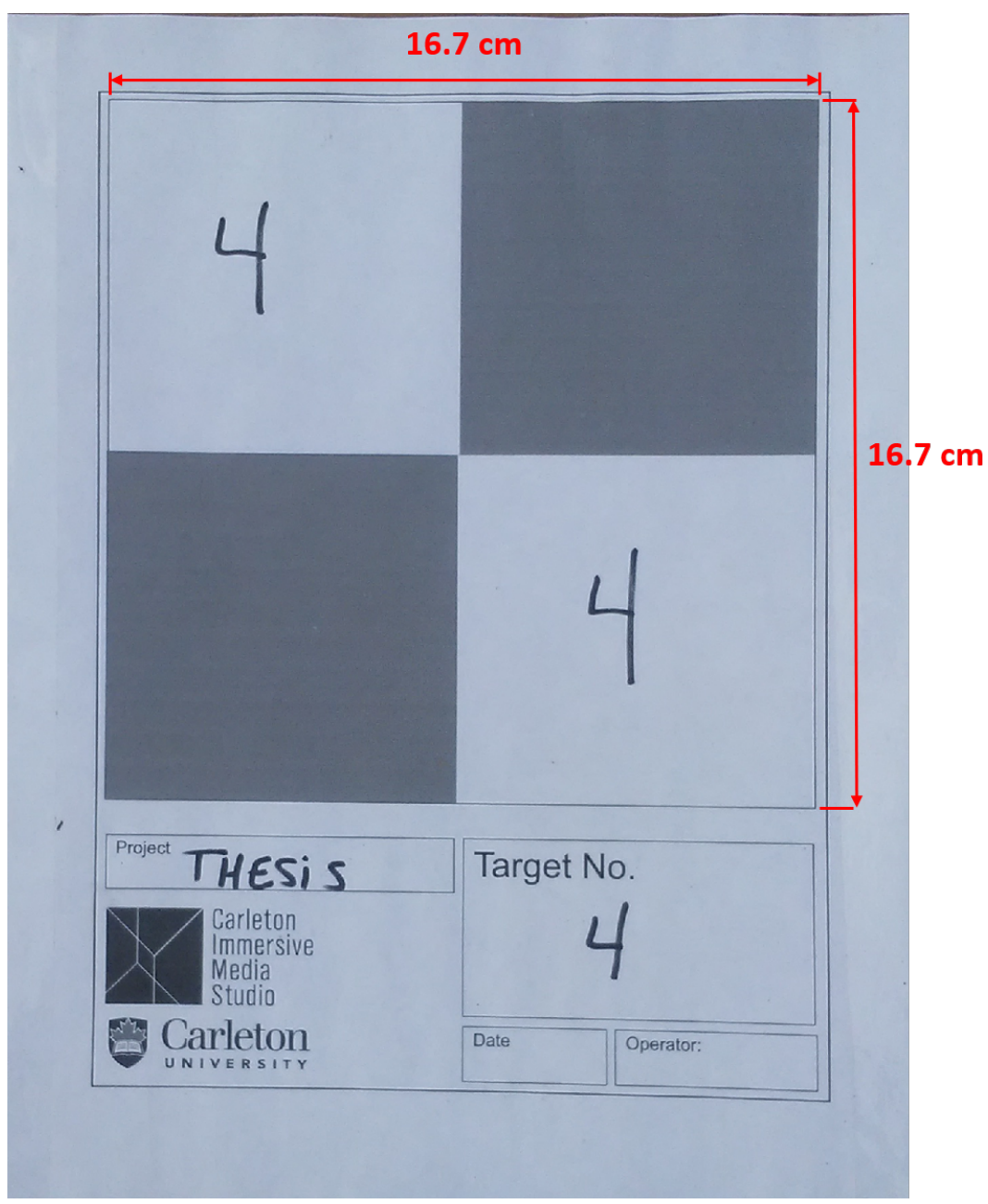

Figure 3.12: Ground control point

To measure the position of each control point, the Emlid Reach real time kinematic (RTK) GPS positioning system was used. The former is a low-cost positioning device which can be powered via micro-USB or DF13 ports and has an operating voltage which ranges from 4.75-5.5 V, with an average current consumption of 200 
mA [51]. The Reach's mechanical properties are summarized in Table 3.3.

Table 3.3: Emlid Reach RTK GPS mechanical properties

\begin{tabular}{|c|c|}
\hline Length & $45.5 \mathrm{~mm}$ \\
\hline Width & $27 \mathrm{~mm}$ \\
\hline Thickness & $9.2 \mathrm{~mm}$ \\
\hline Weight & $14 \mathrm{~g}$ \\
\hline
\end{tabular}

In order to obtain a good signal, there are certain guidelines which should be adhered to for the setup and use of the Reach. The first factor to be considered is the antenna placement; it is recommended that the antenna should have a clear view of the sky at an angle of 30 degrees above the horizon to avoid interference or potential signal blockage [51]. Secondly, the placement of any nearby electronic devices should be considered. Electronic devices may produce radio frequency (RF) noise which can have an adverse effect on the ability to receive a clear GPS signal [51]. Lastly, in order to reduce multipath interference, improve the signal reception, and provide some shielding, the antenna must be placed on a conductive ground plate whose dimensions are no less than $7 \mathrm{~cm} \times 7 \mathrm{~cm}$ [51]. Figure 3.13 shows the Emlid Reach module and antenna. 


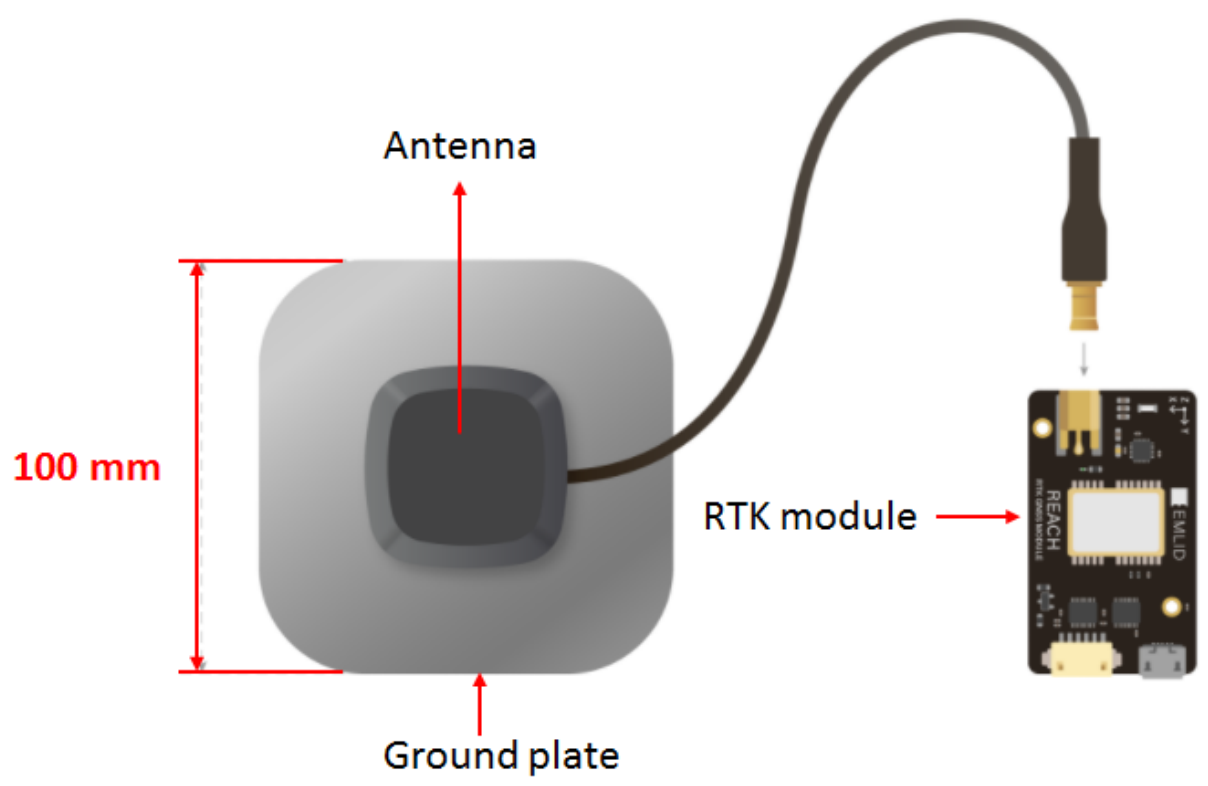

Figure 3.13: Emlid Reach RTK GPS module and antenna [51]

Prior to using the devices for positioning, they must be configured as either a base station or a rover. For positioning with the Emlid Reach RTK system, two modules are needed, one which was configured as the base station while the other was configured as a rover. The steps are described in the supporting documentation [51] and involve connecting to the individual modules via their IP addresses. Figure 3.14 shows the setup of the base station mounted on a camera tripod which was used in the field. 


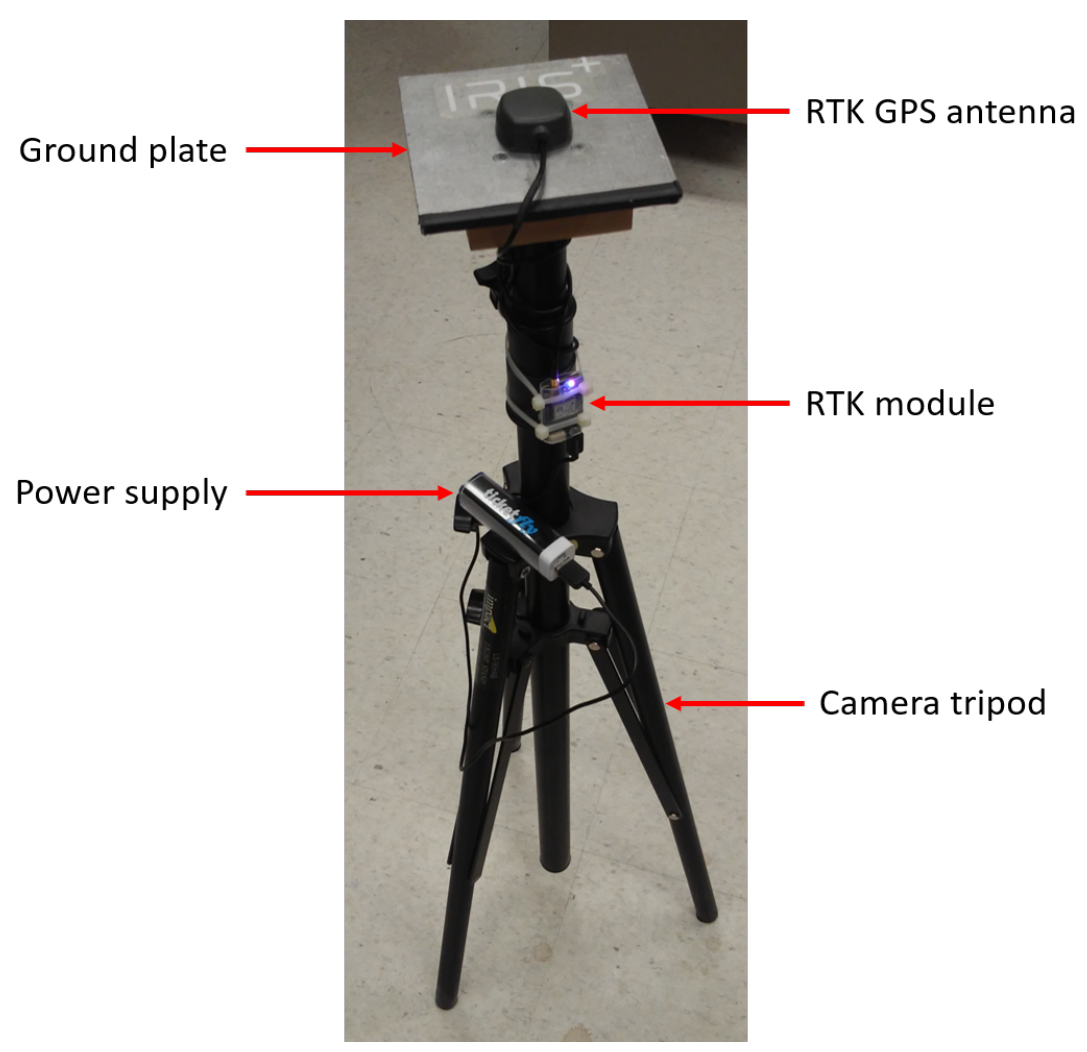

Figure 3.14: RTK GPS mounted on tripod

In the field, once the ground control point targets were placed in their designated positions, the following steps were used to connect to the base station and rover.

1. A Wi-Fi hotspot was created on a mobile device.

2. The laptop was connected to the Wi-Fi hotspot.

3. The base was powered on and given sufficient time for a connection to be achieved. This was shown by a solid green light on the module.

4. Step 3 was repeated for the rover.

5. A tab was opened in an Internet browser and both the base station and rover were connected to the Reachview application through use of their IP 
addresses. The IP address of the rover and base station were 192.168.43.37 and 192.168.43.254 respectively. Once a connection had been established, the data began logging for both devices.

6. Once the experiment had been conducted, the devices were disconnected and the log files were downloaded for the analysis process.

Once a successful connection was established for both the rover and base station, the rover was placed on each ground control point for a period of 5 minutes. This time frame was chosen for two main reasons, the first is that it allowed for a sufficient amount of data points to be gathered, which would later be used to determine the average latitude, longitude and altitude of each reference point. The second reason is due to the availability of the field; there was a limited amount of time to access the field in order to measure the data and 5 minute intervals allowed for the experiment to proceed given the time constraint. At each ground control point, the start time of measurement, the end time as well as the offset distance between the target and antenna on the rover was measured. This information is summarized in Table 3.4.

Table 3.4: Ground control point measurements

\begin{tabular}{|c|c|c|c|}
\hline Position & Start time & End time & Offset [in] \\
\hline First base & $16: 51$ & $16: 56$ & 51.5 \\
& $17: 22$ & $17: 27$ & 51.5 \\
\hline Second base & $16: 57$ & $17: 02$ & 51.25 \\
\hline Third base & $17: 15$ & $17: 20$ & 51.125 \\
\hline Home plate & $17: 09$ & $17: 14$ & 50.75 \\
\hline Pitcher's mount & $17: 03$ & $17: 08$ & 51.875 \\
\hline
\end{tabular}




\subsubsection{Flight 1}

The first of a series of three flights conducted on the River Field was flown in a simple grid configuration at an altitude of $2 \mathrm{~m}$ AGL and at a lateral separation of 2 $\mathrm{m}$ between flight strips. A mission file was constructed using Mission Planner's flight plan option and consisted of 31 distinct waypoints where the point with the label $\mathrm{H}$ represents the takeoff location. Figure 3.15 shows the flight plan.

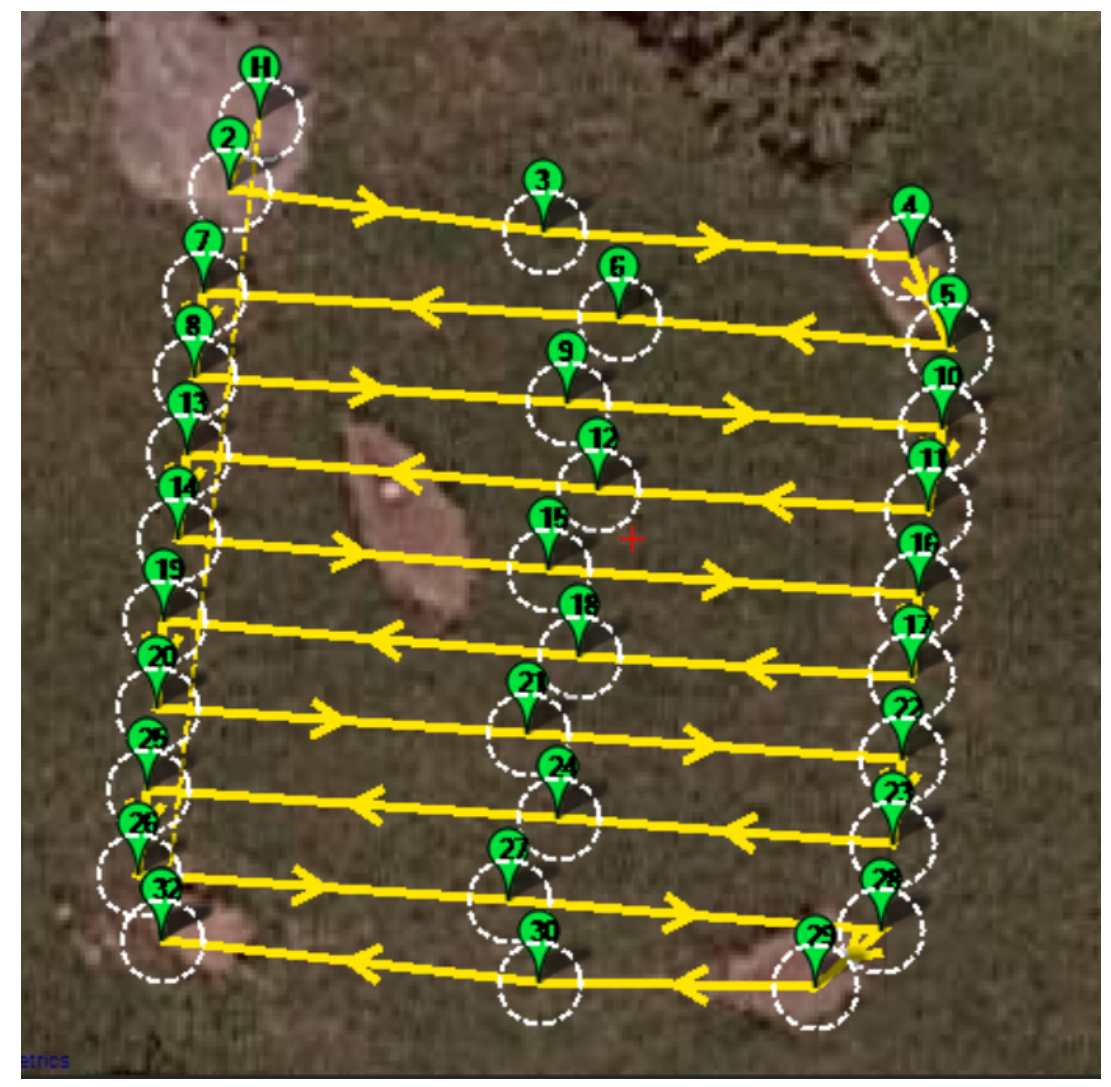

Figure 3.15: First test flight navigation plan

Each waypoint had a hold delay of one second associated to it in order to have a brief pause to prevent the waypoint from skipping over it. The white dashed circles shown in Figure 3.15 represent the waypoint radius, which defines a perimeter that once the UAV crosses considers the current command to be completed and proceeds to the next. Prior to field testing, the mission was simulated using software-in-the-loop 
(SITL) in order to verify that everything functioned properly and that there were no errors in the commands. Furthermore, it allowed the expected behavior of the UAV in ideal conditions to be observed. Initially, the mission was designed with a takeoff and land command programmed into the mission. However, during field testing it was observed that running Dronekit on the Raspberry Pi as well as trying to remotely connect to the Iris+ for a live feed using Mission Planner caused some errors. The solution to this problem was to not remotely connect to the Iris + for an autonomous takeoff, instead the Iris + took off from the ground manually then the vehicle mode was switched to AUTO and the mission started. Once the UAV had completed its final waypoint, the landing command was triggered and the Iris + descended at that point.

\subsubsection{Flight 2}

The second flight consisted of a simple grid configuration flown at an altitude of 3 $\mathrm{m}$ relative to the ground level and with a distance of $2 \mathrm{~m}$ between flight strips. A mission file was constructed using Mission Planner's flight plan option and consisted of 37 distinct waypoints where the point with the label $\mathrm{H}$ represents the location of the takeoff. Figure 3.16 shows the flight plan. 


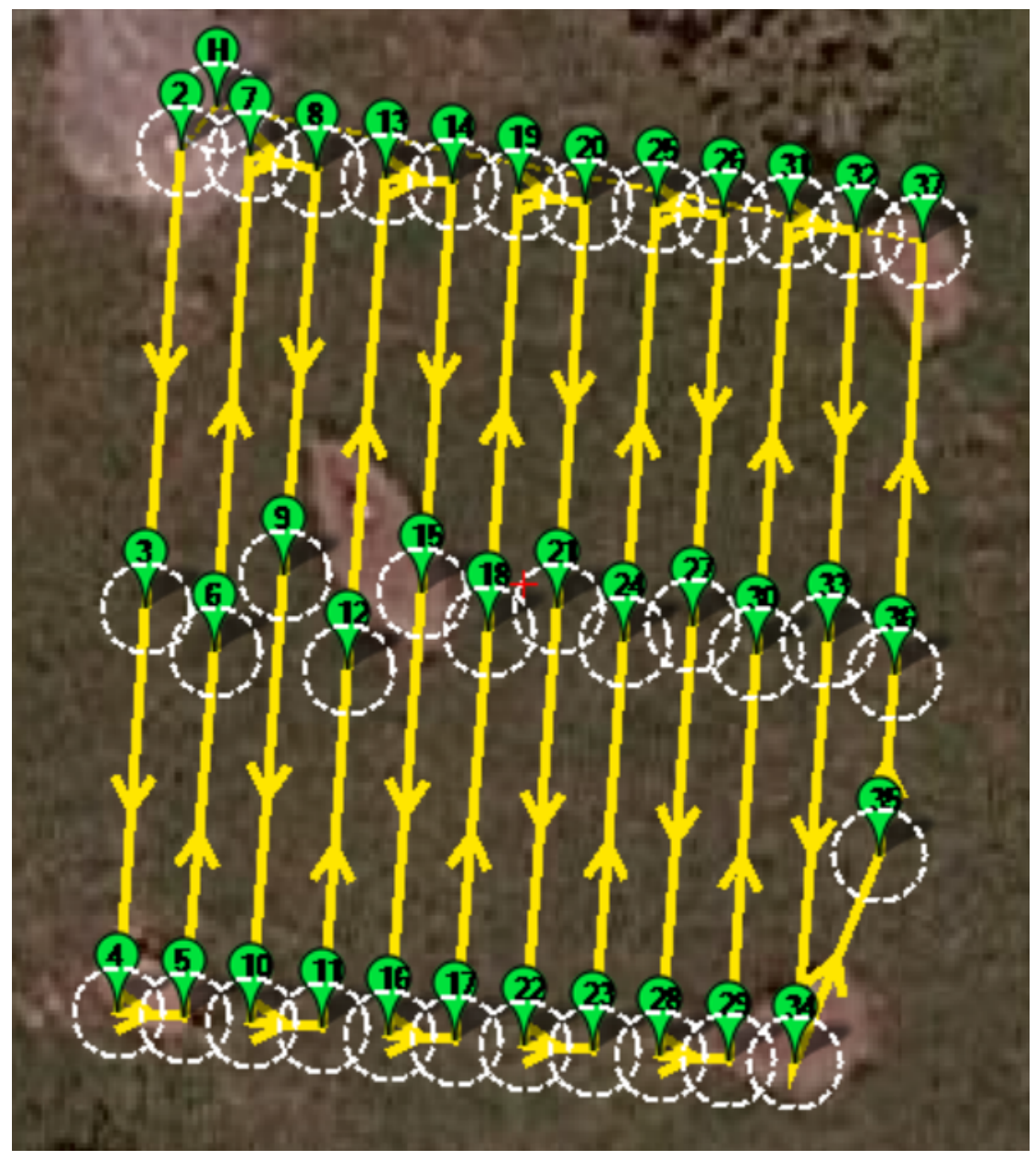

Figure 3.16: Second test flight navigation plan

The configuration of the individual waypoints were the same as in flight 1. During the later portion of flight 2, manual control of the UAV had to be assumed and the UAV landed as it approached surrounding trees. This was necessary step to avoid collision. Once the UAV had been safely recovered, it was brought back to the bench and the mission plan was modified in order to avoid the trees. It is important to note that only the last few waypoints were affected and thus the images and data gathered were still usable. The modified flight plan is shown in Figure 3.17. 


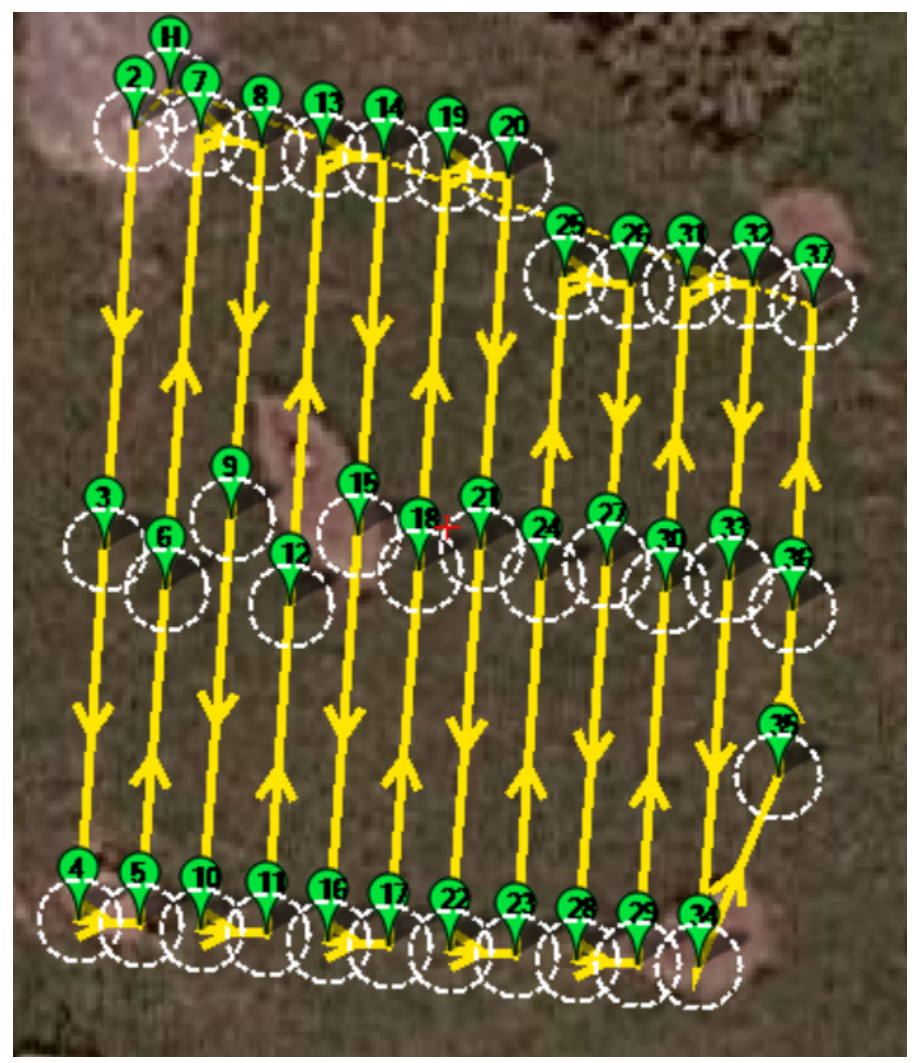

Figure 3.17: Modified navigation flight plan for second test 


\section{Chapter 4}

\section{Results and Discussion}

The objective of this research was to investigate an alternative cost-effective means of direct georeferencing and data acquisition for small UAV systems using the available onboard sensors. To this end, adding additional computational functionality onboard the UAV was explored. In order to meet this objective, a custom data acquisition script was developed using Dronekit and a Raspberry Pi single board computer was placed onboard the aircraft in order to communicate with the Pixhawk autopilot.

On August 20th, 2017, an aerial survey was conducted over River Field, located on the Carleton University campus. The survey consisted of three separate flight passes which acquired 215, 250 and 249 images and data points, respectively. This chapter presents and discusses the results for the experiments summarized in Chapter 3. Firstly, a comparison between the Pixhawk's telemetry log and the custom data acquisition script that was developed will be presented. Lastly, the georeferencing results, which include a comparison of the predicted and calculated values for the exterior orientation parameters as well a comparison of the generated 3D point clouds for each method of georeferencing will be explained. 


\subsection{Data Acquisition Script}

Through the use of Dronekit, a custom data acquisition Python script was developed in order to directly associate the relevant telemetry data to the respective image being captured in real time. During flight, each time an image is captured, the position information of the UAV is saved in the format of longitude, latitude and altitude above-sea-level (ASL). Furthermore, the attitude (roll, pitch and yaw) of the UAV is also logged in radians. Lastly, a timer was included in the script to determine total flight time and aid in post-flight graphing of results.

A comparison between the recorded data from the Pixhawk's telemetry log and the custom data acquisition script was conducted to ensure that the developed script functioned and logged the data properly. Moreover, this comparison also allowed for the differences in how the information is logged between both methods to be highlighted. It is important to note that only the data from flight 3 (which is the modified flight plan from Section 3.2.2.4) will be used for the analysis. The former is primarily due to the manner in which the Pixhawk records the flight data. Once the UAV is powered, the Pixhawk will continuously log data until either the battery dies or the UAV is powered down. Since the first flights were conducted on one battery life, it becomes very difficult to distinguish both flights in a very large data file. However, prior to the third flight, the battery was changed, therefore the data logged for that test corresponded to only that flight, which allowed for the data to be used. Initially, the recorded positions of the UAV using the Pixhawk's telemetry logs were compared to the values obtained from the custom script, the results are shown in Figure A.5. 


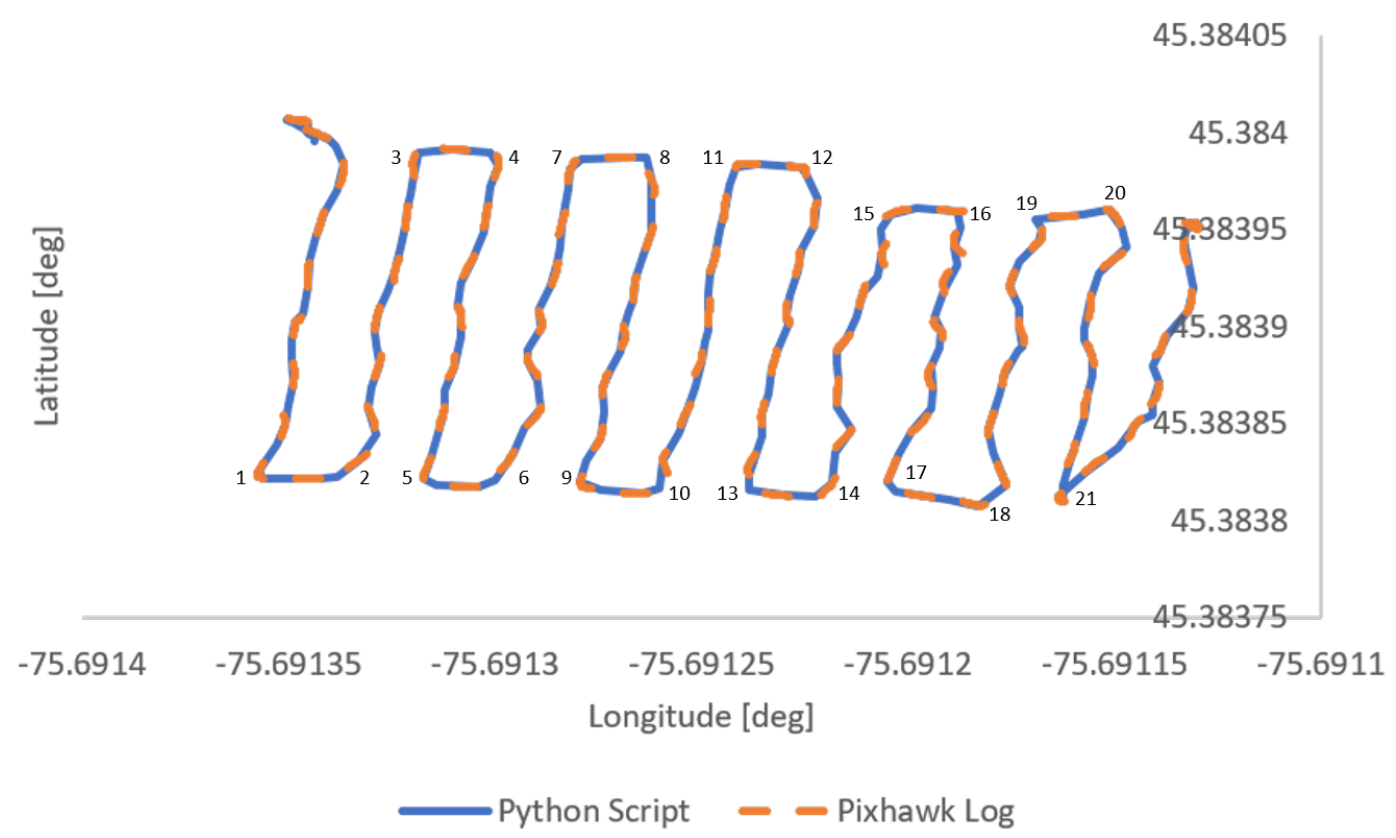

Figure 4.1: Comparison between the UAV's position logged by the Pixhawk and the custom Python data acquisition script

Observing Figure A.5 it can be seen that both the results recorded from the custom script as well as the Pixhawk's match and have virtually the same profile with very minor deviations. Figure 4.2 shows the comparison between the recorded values for the altitude of the UAV (ASL) as a function of time of flight. 


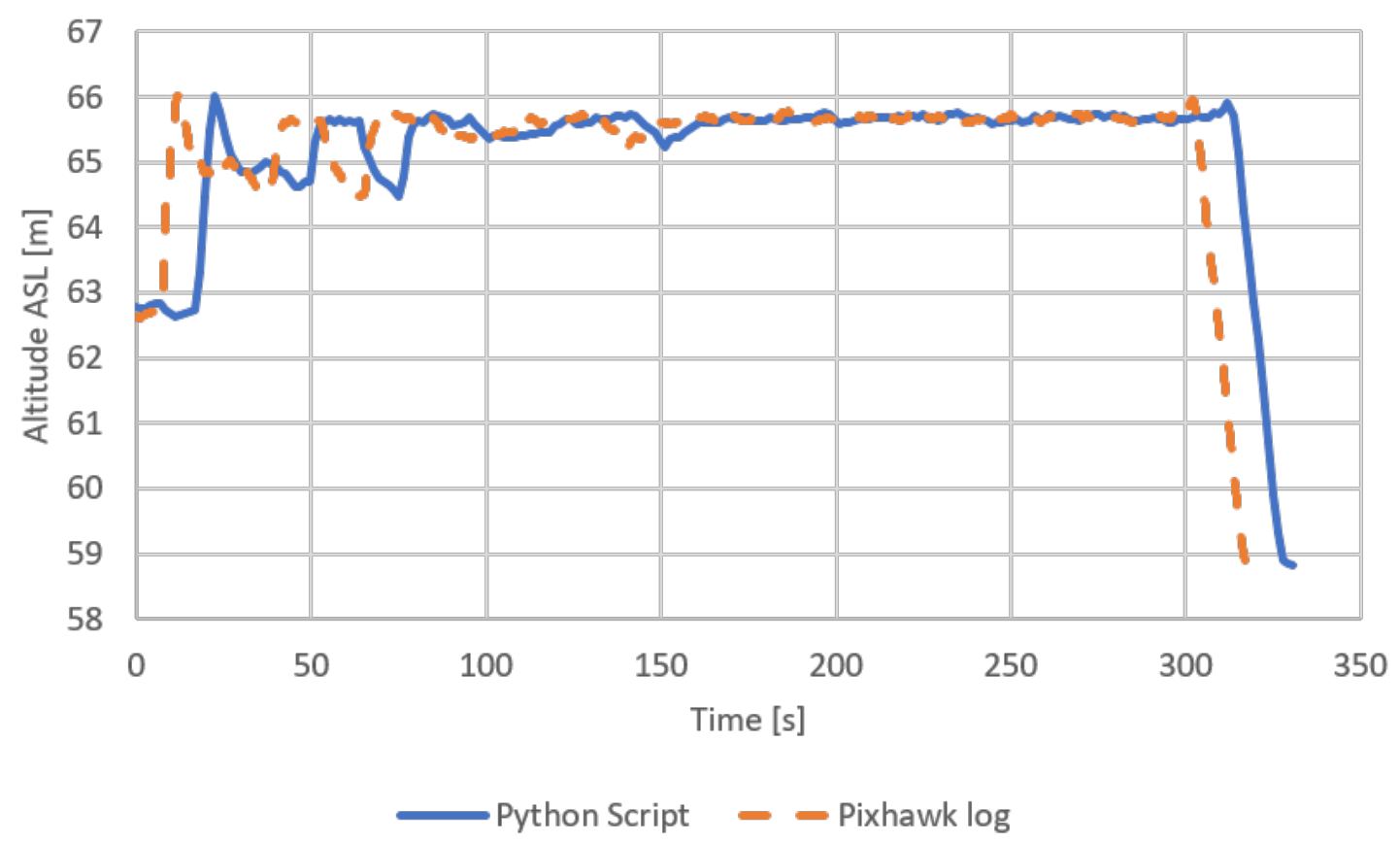

Figure 4.2: Comparison between the UAV's altitude logged by the Pixhawk and the custom Python data acquisition script

Upon further inspection, it can be seen that the results for both recording methods follow a very similar profile; however, they appear to be offset in time. The value of the offset between both data sets was determined to be 7 seconds. The reason that there is an offset present can be explained by the manner in which both methods record the data. As previously stated, once the UAV is powered, the Pixhawk immediately begins to log data while the custom script will only begin to log data once the code begins to run, which is just before flight and at the discretion of the user. Furthermore, once the script starts to run there is a delay between the run command and the time the script begins to log data. The former is due to the fact that the code begins by attempting to connect to the vehicle which is followed by the creation of the data folders and the initialization of the camera, which takes time. The corrected graph which takes into account the offset is shown in Figure 4.2. 


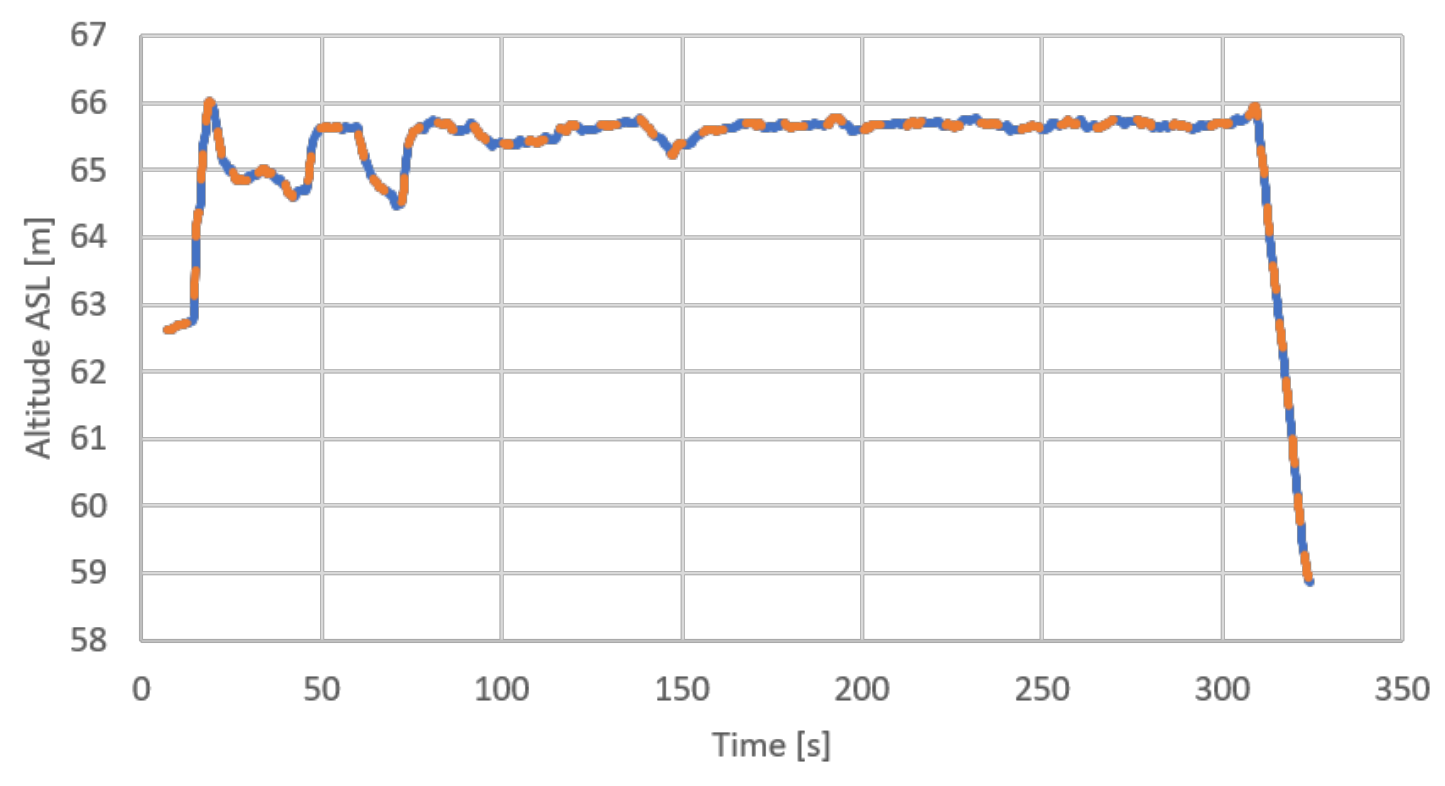

Python Script - Pixhawk Log

Figure 4.3: Comparison between the UAV's altitude logged by the Pixhawk and the custom Python data acquisition script corrected

Once the dataset from the Pixhawk's telemetry log was shifted it could be seen that the results for both recording methods matched with no deviations. The results shown in both Figures A.5 and 4.3 show that the custom data acquisition script developed using Dronekit functions properly when recording positional information.

Upon further analysis of Figure 4.3, it can be noted that there are some major variations in altitude at the beginning of the mission. As stated in Section 3.2.2.3, due to complications when running Dronekit while trying to employ a live connection to Mission Planner, The UAV's takeoff was done manually then the vehicle's mode was changed to AUTO. At this point the UAV adjusts its altitude to the specified value set in the mission parameters. For Flight 2, the mission's altitude was set to 3 meters above-ground-level (AGL). Initially, the recordings show the UAV at an altitude of approximately $62.5 \mathrm{~m}$ ASL and it appears that the altitude begins to stabilize after 
75 seconds at which point the values deviate close to an approximate value of $65.5 \mathrm{~m}$. Since the specified mission altitude was $3 \mathrm{~m}$ AGL, the UAV must constantly adjust its altitude to the terrain which could account for the deviations noted in Figure 4.3.

The second characteristic that was used to gage whether the custom data acquisition script functioned properly was to compare the orientation data of the UAV logged by the script with the results of the Pixhawk's telemetry log. Figure 4.4 shows the roll angle of the UAV in radians plotted against the time of flight. 


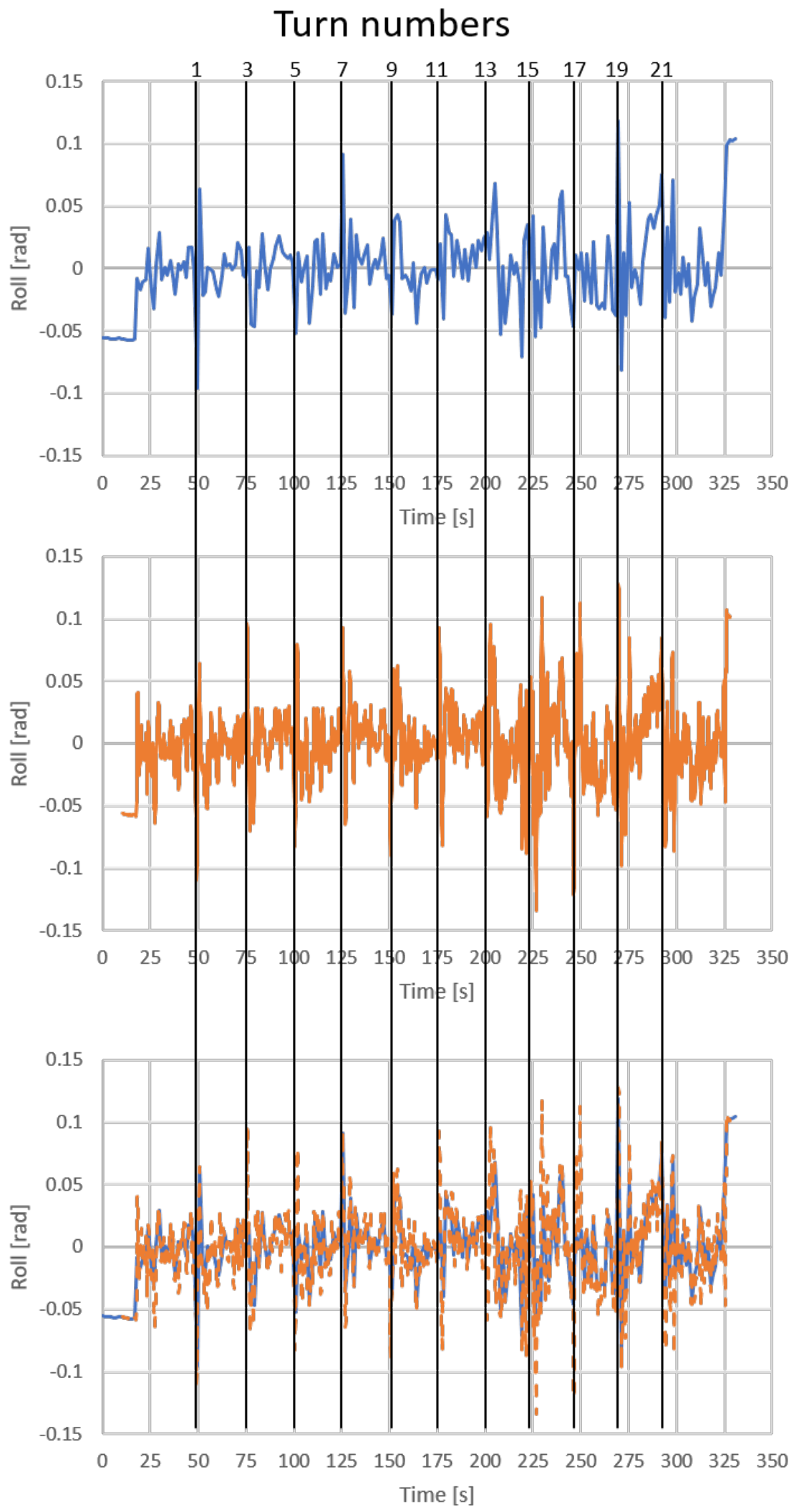

Figure 4.4: Comparison between the UAV's roll logged by the Pixhawk and the custom Python data acquisition script showing turn numbers 
The graph on the top of the figure corresponds to the data acquired by the custom data acquisition script, the graph in the middle corresponds to the data logged by the Pixhawk. Lastly, the graph located on the bottom of Figure 4.4 shows the comparison of the two different methods. The vertical lines which pass through the graphs identify specified points along the flight path and are labeled in the same order as shown in Figure A.5. Upon further inspection of Figure 4.4 and comparing the values of the peaks and the labeled points, it can be seen that the data acquisition script and the Pixhawk's telemetry log are in agreement at points 1, 7, 13 and 15 while they are in disagreement at points 3, 5, 9, 11 and 17 . However, at points 19 and 21 , both methods show the same profile except that the peak values vary slightly. Figure 4.5 shows the pitch angle of the UAV in radians plotted against the time of flight. 
Turn numbers

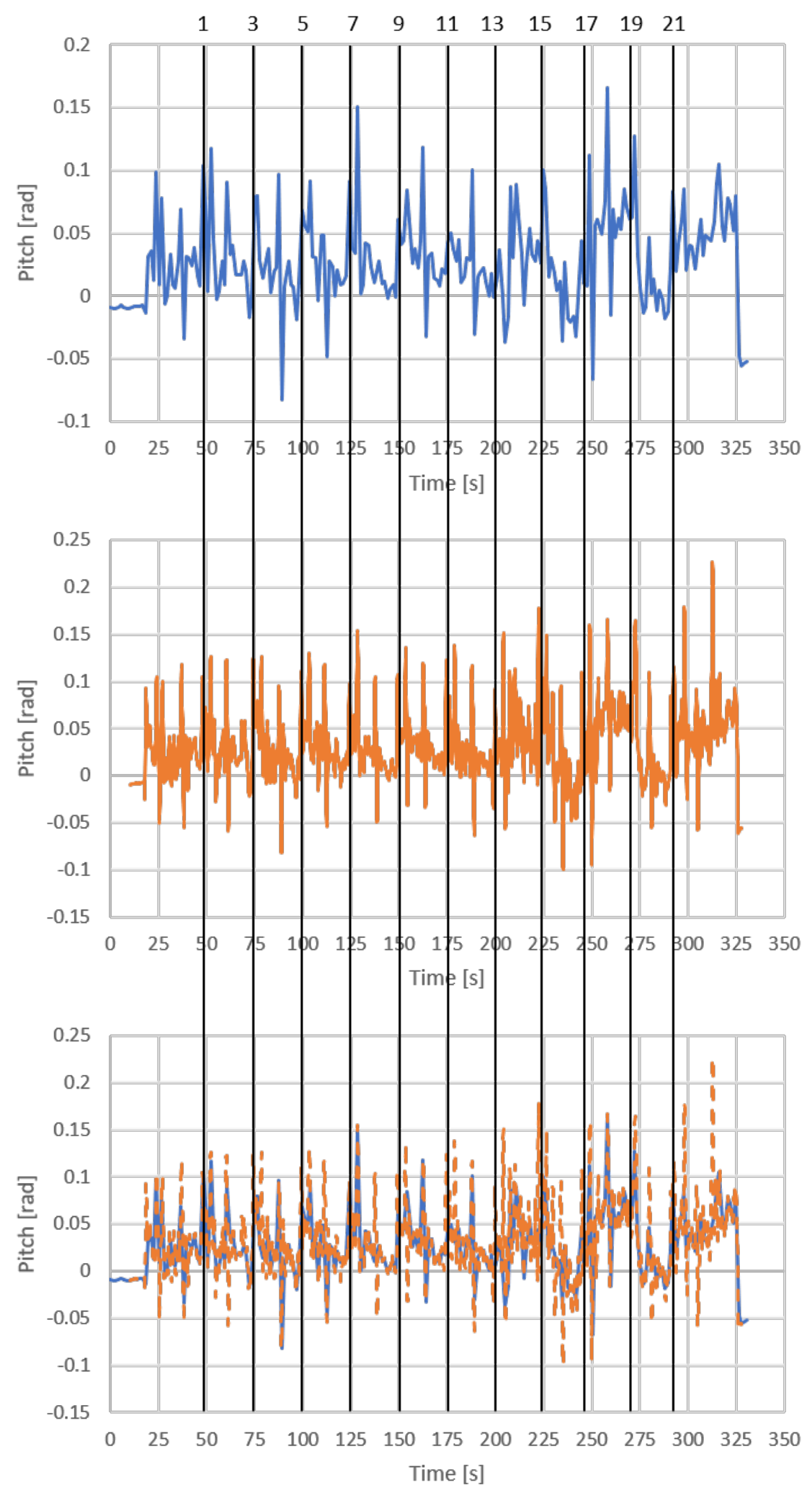

Figure 4.5: Comparison between the UAV's pitch logged by the Pixhawk and the custom Python data acquisition script showing turn numbers 
The comparison of the pitch angle of the UAV for the specified points along the flight path showed agreement at points 1, 15, and 19 while there was disagreement between the values at points 9, 11, 13, 18. However, for the case shown in Figure 4.5, points 3, 5, 7 and 21 showed the same profile for both the data acquisition script and telemetry log with minor variances in the maximum/minimum peak values. Considering the results presented for the recording of both the roll and pitch of the $\mathrm{UAV}$, it can be said that the data logged by the script does not agree with the data logged by the Pixhawk $40 \%$ of the time while $60 \%$ of the time the data matches or are very similar with minor differences. Another difference which is important to highlight is that the sampling rate of both methods differ. In the data acquisition script, telemetry data is only gathered at capture time, which was $1 \mathrm{~Hz}$ for the tests discussed in this section. However, the Pixhawk captures data at a rate of approximately $10 \mathrm{~Hz}$, thus it is possible to have certain sampling errors. Figure 4.6 shows the yaw angle of the UAV in radians plotted against the time of flight. 


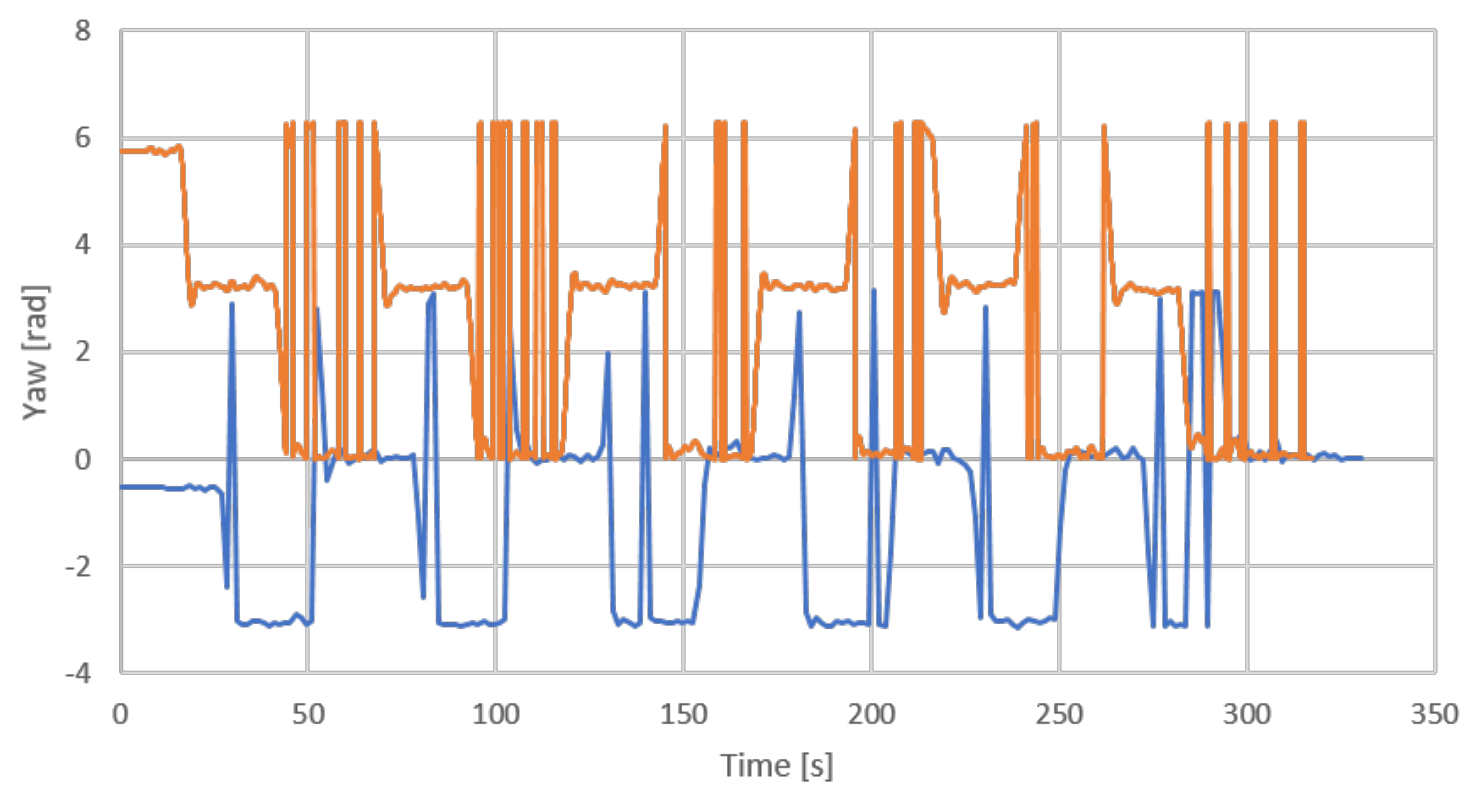

Python Script —Pixhawk Log

Figure 4.6: Comparison between the UAV's yaw logged by the Pixhawk and the custom Python data acquisition script

The data shown in Figure 4.6 enabled the principle difference between both data logging methods to be highlighted. The Python data acquisition script recorded the yaw angle relative to initial position of the UAV while the Pixhawk logs the data in an absolute reference frame. The value of the yaw angle is zero when the UAV is facing North and its angles are recorded from 0 to $2 \pi$ while the Python records angles from $-\pi$ to $\pi$. The former is one of the main reasons for the differences which can be observed in Figure 4.6. The second noticeable difference between both datasets is that the Pixhawk's shows more variations compared to its counterpart, the Python script. The profile of the script was more in line with the expected profile based on the flight plan. As seen in Figure A.5, it can be noted that travel between points is not done in a perfectly straight line, but there are visible perturbations to the flight path. Perturbations that would normally occur in the negative direction are recorded 
as such by the Python script; however, the Pixhawk records them with reference to the positive direction thus small deviations that occur during flight are represented as large deviations in Figure 4.6.

\subsection{Georeferencing Results}

As previously stated, a series of three separate flight passes were used for image acquisition with the objective of producing a 3D point cloud for each series of images. The first flight pass, see Section 3.2.2.3, was flown along the width of the field and acquired 215 images and data points with an operating time of approximately 310 seconds. The second flight pass, see Section 3.2.2.4, was flown along the length of the field, acquiring 250 images and data points with an operating time of 355 seconds. As stated in Chapter 3, during this flight the UAV had to be manually taken over to avoid collision with the surrounding tress. However, this step occurred towards the end of the trial and thus the data that had been gathered was still usable. The previous flight plan was modified and a third flight pass was conducted which acquired 250 images and data points with an operating time of approximately 357 seconds.

The objective of this thesis was to improve the current method of conducting aerial photogrammetry through added onboard computer functionality. A custom data acquisition script was developed in order to directly associate the relevant telemetry information to the image being captured in real time. This step could improve on the current methods of direct georeferencing. The former, as discussed in Section 2.3.3, is a technique which aims to calculate the parameters of exterior orientation using data from onboard sensors, specifically GPS/INS and inertial measurements sensors. Based on the research conducted in Chapter 2, it was determined that the 
synchronization between the image capture times and the GPS/telemetry logs is of vital importance to the direct georeferencing process. Upon further investigation, the commonly employed solution to the synchronization component was determined to be using additional onboard components to accurately determine the capture time to compare with logged GPS and IMU data during post-processing. However, this process requires a high degree of precision and additional time for post-processing of the results. The proposed system in this thesis aimed to present an alternative to this commonly used method through the use of onboard and low-cost sensors.

Using the data gathered during each of the flights, a MATLAB script was used to calculate the parameters of exterior orientation of the camera station for each acquired image. The images were then processed using Agisoft's PhotoScan Professional Edition version 1.2.6 in order to generate detailed 3D point clouds for analysis. Three different point clouds were generated; one for each method of georeferencing. Keeping with the method for verification and validation for DG systems identified through literature, an initial 3D point cloud was generated using the indirect method of georeferencing, which employed ground control points. This model was then used as an absolute reference and compared to the 3D point clouds generated using the direct and assisted georeferencing methods. The 3D point cloud which was generated from the images captured during the first flight is shown Figure 4.7. 


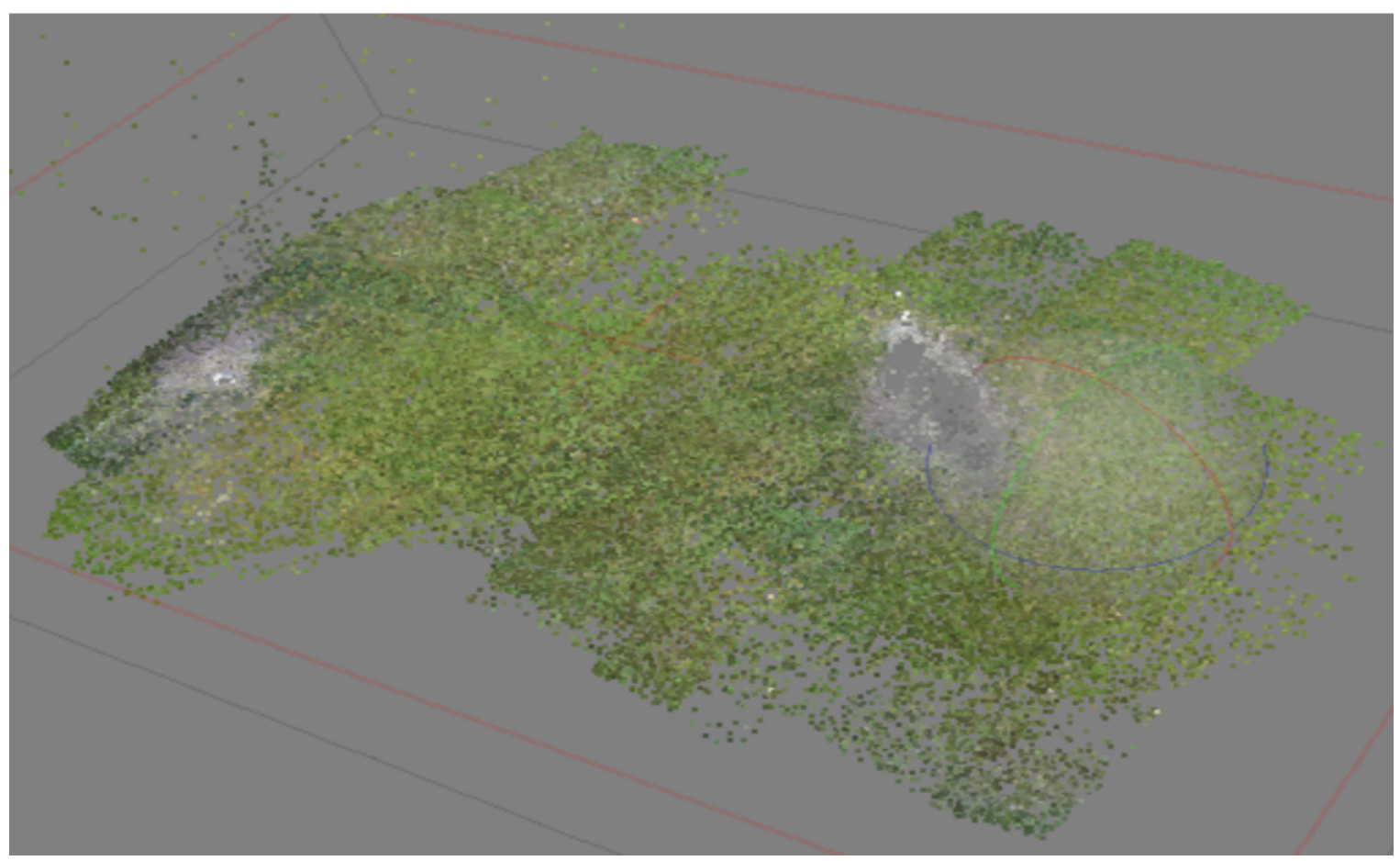

Figure 4.7: Initial generated 3D point cloud using data from flight test 1

Originally the process mentioned above was going to be conducted for each test flight; however, once the 3D point cloud was generated for the first flight, it was observed that the entire field was not captured and that during PhotoScan's photo alignment process, a majority of cameras did not align. The former could be due to poor resolution of the image or that there was not enough overlap or sidelap between flight strips. It is for this reason that all the images and data collected for all three flights were processed at once. This produced a much more intricate flight path and greatly increased the amount of overall sidelap and overlap between images. The combined flight paths are shown in Figure 4.8 . 


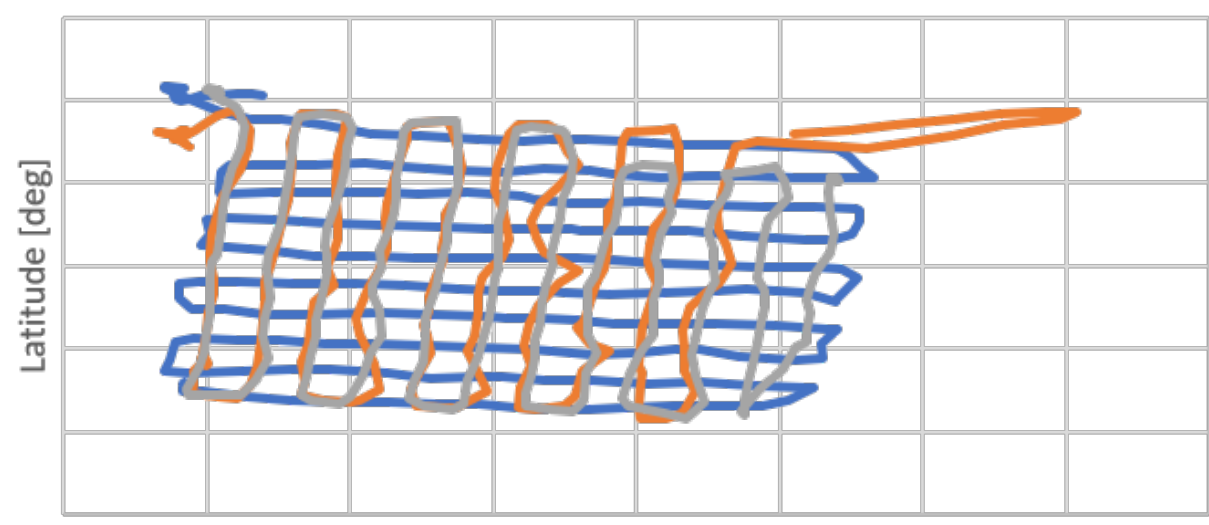

Longitude [deg]

Flight 1 Flight $2=$ Flight 3

Figure 4.8: Combined flight paths.

Once all the images were processed, they produced a much more detailed 3D point cloud and included the whole surveyed field. For the analysis, three 3D point clouds were generated for the combined data; using indirect, direct and assisted georeferencing.

\section{Indirect Georeferencing}

As discussed in Chapter 2, the indirect method of georeferencing treats the parameters of exterior orientation as an unknown which needs to be determined and is estimated through aerial triangulation, which requires the use of ground control points. For the survey conducted on River Field, five ground control points were used and their positions were measured using the Emlid Reach RTK positioning device, which was discussed in Chapter 3. Using this data, a georeferenced dense 3D point cloud was generated, which is shown in Figure 4.9. 


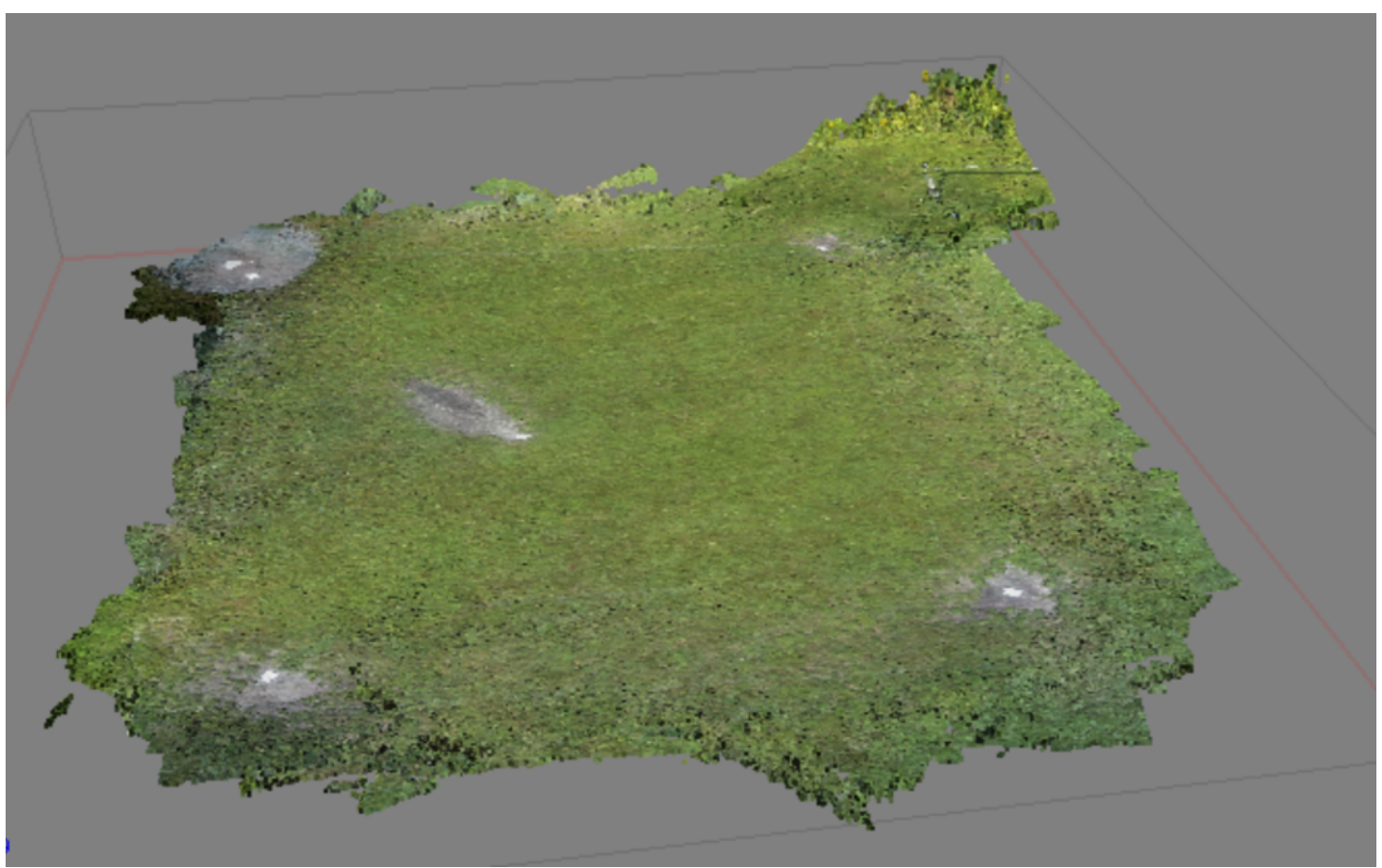

Figure 4.9: 3D point cloud generated using the indirect method of georeferencing.

A georeferenced point cloud is generated in PhotoScan if either the position of the camera or the ground control points are given in a global reference frame. The accuracy of the generated point cloud is governed by the accuracy of the measurements of the coordinates for either the ground control points or exterior orientation parameters. For the indirect georeferenced 3D point cloud, the Emlid Reach RTK positioning device, which can provide centimeter level accuracy, was used to measure the position of each of the ground control points. Using the gathered data, the average position of the ground control was determined in the World Geodetic System 1984 (WGS84) and then converted to the Universal Transverse Mercator (UTM) coordinate system using a conversion function obtained from the geodetic toolbox extension for MATLAB. The average position of the ground control points are listed in Table 4.1. 
Table 4.1: Average position of the ground control points

\begin{tabular}{|c|c|c|c|}
\hline Ground Control Points & Easting [m] & Northing [m] & Altitude ASL [m] \\
\hline First Base & 445876.83 & 5025822.93 & 56.73 \\
\hline Second Base & 445890.24 & 5025821.41 & 56.68 \\
\hline Third Base & 445892.34 & 5025837.88 & 56.58 \\
\hline Home Plate & 445876.61 & 5025840.69 & 56.59 \\
\hline Pitcher's Mount & 445881.77 & 5025830.44 & 56.64 \\
\hline
\end{tabular}

The altitudes provided in Table 4.1 are ASL. However, the altitude given by the Reach is WGS84 ellipsoidal height, thus conversion from ellipsoidal height to altitude abovesea-level was required. A visual representation of the different height conventions is shown in Figure 4.10.

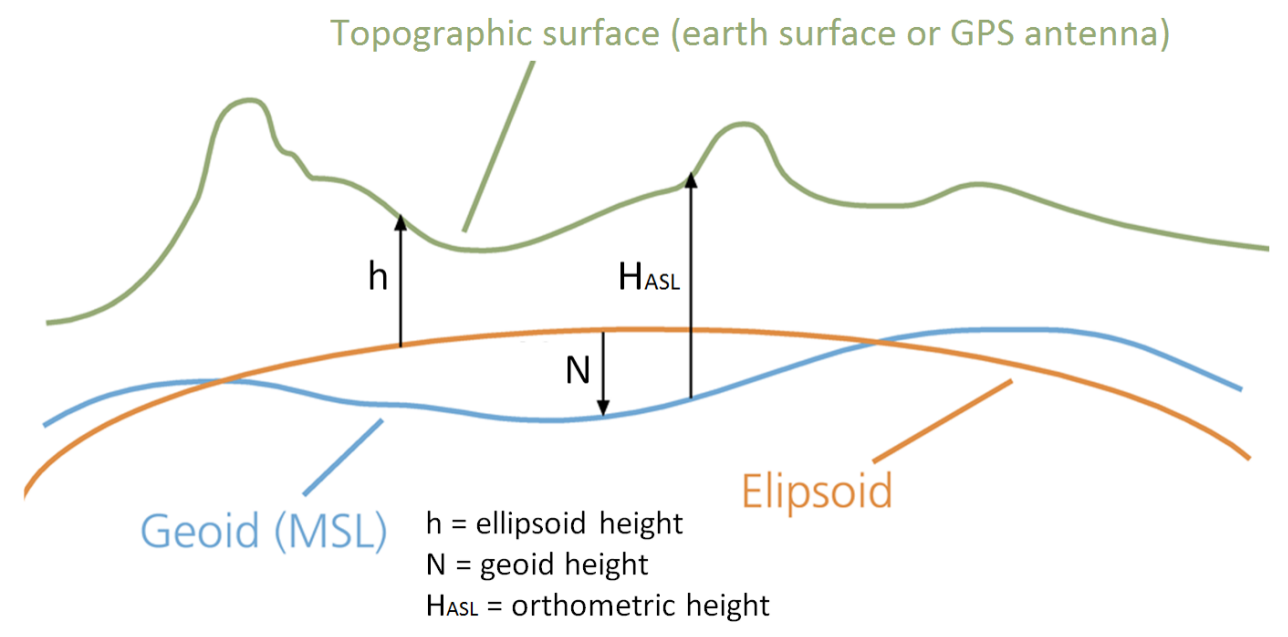

Figure 4.10: Visual representation of different height measurement conventions [52]

As previously stated, the ellipsoidal height was given by the Reach in meters. 
The geoid height was determined using the built-in MATLAB function geoidheight, which requires the latitude and longitude of the point of interest as inputs and outputs the geoid height in meters. Lastly, the altitude above-sea-level, also called the orthometric height is determined by subtracting geoid height from the ellipsoid height. This is shown in Equation 4.1.

$$
H_{A S L}=h-N
$$

The data listed in Table 4.1 was imported into PhotoScan and used to produce the point cloud shown in Figure 4.9. Once the point cloud was finished being built, the software's calculated exterior orientation parameters were exported to a text file and used as an absolute reference for comparison with the 3D point clouds generated through direct and indirect georeferencing.

\section{Direct Georeferencing}

Direct georeferencing aims to calculate the exterior orientation parameters using data from the onboard sensors of an aircraft. The ability to directly determine the exterior orientation parameters of the camera station at the capture time is the fundamental difference between the indirect and direct methods of georeferencing. For the system outlined in this thesis, the problem can be described as a series of vector additions, subtractions as well as coordinate transformations. This is illustrated in Figure 4.11. 


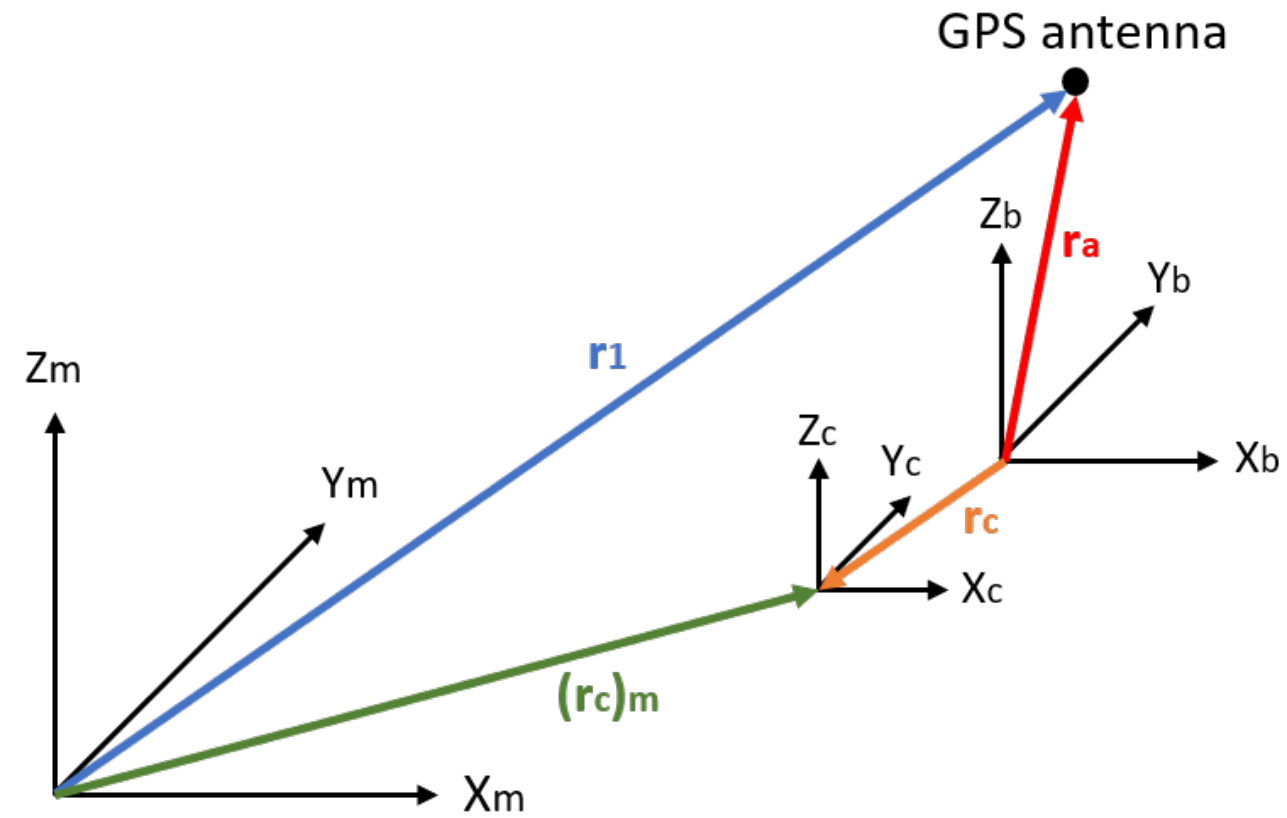

Figure 4.11: Direct georeferencing vectors.

where the subscript $m$ represents the mapping frame, the subscript $b$ represents the body frame and the subscript $c$ represents the camera's frame of reference. Given this problem, the objective is to determine an expression for the coordinates of the camera's perspective center in the global (i.e. mapping) reference frame and is accomplished using Equation 4.2.

$$
\left(r_{c}\right)_{m}=r_{1}+\mathbf{R}_{b}^{m}\left[-r_{a}+r_{c}\right]
$$

where:

- $r_{1}$ represents the coordinate vector of the navigation sensors (GPS) in the mapping frame

- $r_{a}$ represents the coordinate vector between the IMU's center and the antenna, 
in the body frame

- $r_{c}$ represents the coordinate vector between the IMU and the camera, in the body frame

- $\left(r_{c}\right)_{m}$ represents the vector to the camera's perspective center in the mapping frame

The term $\mathbf{R}_{b}^{m}$ represents the 3 x 3 rotation matrix used to align the body and mapping frame. The former is built using the attitude information of the aircraft at the image capture time. Let $\alpha$ be the roll of the aircraft, $\beta$ be the pitch of the aircraft and $\gamma$ be the yaw. The rotation matrix is then determined using Equation 4.3

$$
\mathbf{R}_{b}^{m}=\left[\begin{array}{ccc}
1 & 0 & 0 \\
0 & \cos \alpha & -\sin \alpha \\
0 & \sin \alpha & -\cos \alpha
\end{array}\right]\left[\begin{array}{ccc}
\cos \beta & 0 & \sin \beta \\
0 & 1 & 0 \\
-\sin \beta & 0 & \cos \beta
\end{array}\right]\left[\begin{array}{ccc}
\cos \gamma & -\sin \gamma & 0 \\
\sin \gamma & \cos \gamma & 0 \\
0 & 0 & 1
\end{array}\right]
$$

Prior to calculating the position of the camera's perspective center using Equation 4.2 certain preparatory steps needed to be completed. Firstly, measurements needed to be taken on the UAV. Specifically, the position of the GPS antenna and camera relative to the center of the IMU in all three planes needed to be determined. Measurements were taken from a fixed point of reference which was aligned with the rear of the UAV and values were obtained using a digital caliper with an accuracy of $0.01 \mathrm{~mm}$. However, there were certain measurements that could not be taken with the calipers and thus a metric measuring tape, which had an accuracy of $1 \mathrm{~mm}$, was used instead. All measurements taken were recorded three times and the average 
values were used. The calculated position of the GPS antenna and camera relative to the IMU are summarized in Table 4.2.

Table 4.2: Position coordinates relative to the UAV's IMU

\begin{tabular}{|c|c|c|}
\hline Position & Antenna & Camera \\
\hline $\mathbf{x}[\mathbf{c m}]$ & 4.80 & 1.52 \\
\hline $\mathbf{y}[\mathbf{c m}]$ & 0.38 & -0.93 \\
\hline $\mathbf{z}[\mathbf{c m}]$ & 2.34 & -12.55 \\
\hline
\end{tabular}

The second step to be completed prior to calculating the position of the camera's perspective center was to convert the positional information recored by the Dronekit script from World Geodetic System 1984 (WGS84) to the Universal Transverse Mercator (UTM) coordinate system. This was done using a function obtained from the geodetic toolbox available in MATLAB. Using the converted data in combination with the values from Table 4.2, the MATLAB script calculated the position of the camera's perspective center for each image taken during the survey. The results were then exported to a text file and imported into PhotoScan prior to the photo alignment process. The generated 3D point cloud is shown in Figure 4.12. 


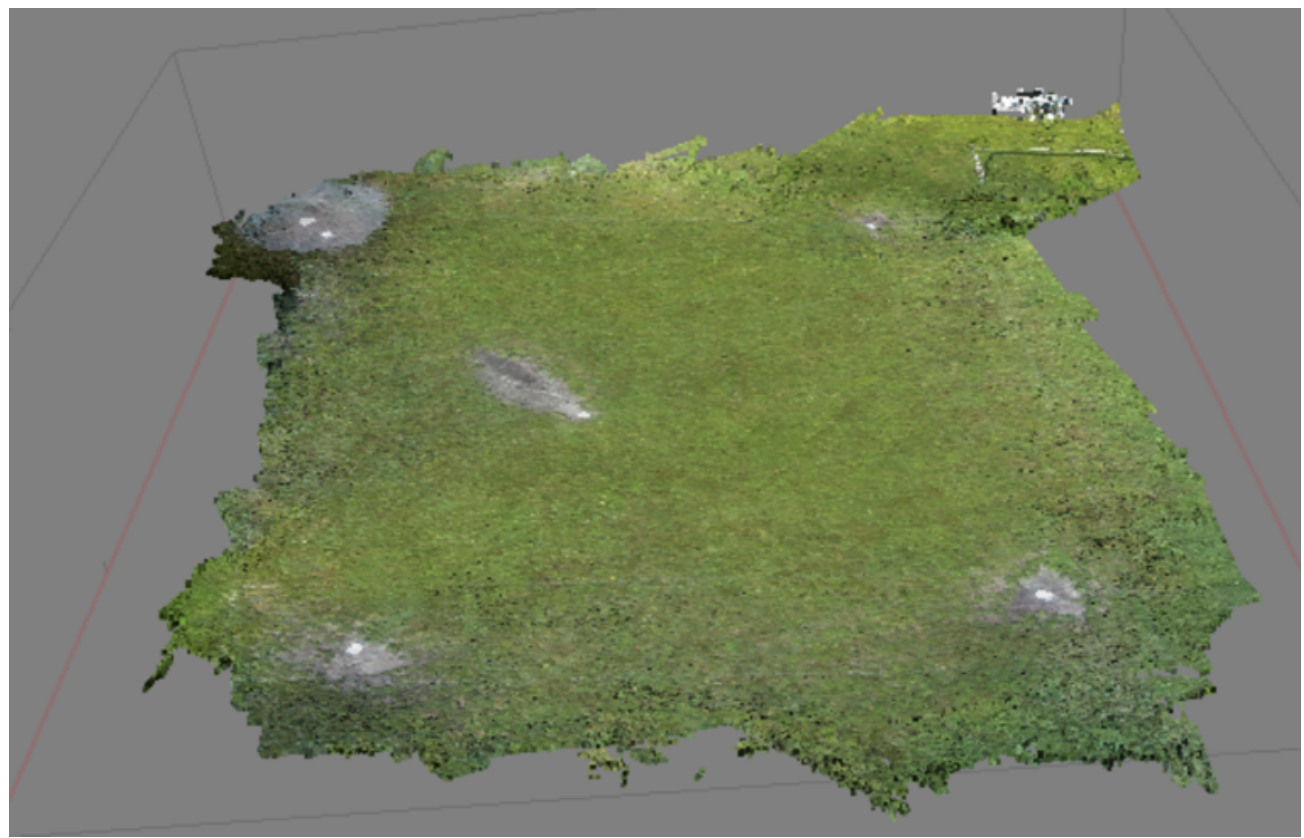

Figure 4.12: 3D point cloud generated using the direct method of georeferencing.

There were two methods employed for the verification and validation of the proposed system; the first was to compare the calculated values for the parameters of exterior orientation obtained using the data from the custom script with those predicted by PhotoScan for the indirect point cloud using the export camera feature. The second method was to directly compare the generated 3D point clouds for the direct and assisted methods with the indirect georeferencing's point cloud. Both these methods are discussed in the later sections of this chapter.

The exterior orientation parameters determined from the indirect georeferenced 3D point cloud were exported and compared to the values obtained from the direct georeferenced 3D point cloud. Table 4.3 summarizes the average difference between the predicted (i.e. indirect method) and calculated (i.e. direct method) results organized by flight. 
Table 4.3: Absolute averaged difference between indirect and direct results

\begin{tabular}{|c|c|c|c|}
\hline Flight & Easting $[\mathbf{m}]$ & Northing $[\mathbf{m}]$ & Altitude [m] \\
\hline $\mathbf{1}$ & 0.372 & 0.507 & 1.724 \\
\hline $\mathbf{2}$ & 0.398 & 0.807 & 1.692 \\
\hline $\mathbf{3}$ & 0.376 & 0.515 & 1.763 \\
\hline Average $[\mathbf{m}]$ & $\mathbf{0 . 3 8 2}$ & $\mathbf{0 . 6 1 0}$ & $\mathbf{1 . 7 2 6}$ \\
\hline
\end{tabular}

\section{Assisted Georeferencing}

As discussed in Chapter 2, the assisted method of georeferencing combines features from both the direct and indirect methods to determine the parameters of exterior orientation. The methods uses the exterior orientation parameters determined using the data from the onboard sensors as initial estimates for the aerial triangulation process. When creating the $3 \mathrm{D}$ point cloud for this case, both the data calculated using the MATLAB script as well as the measurements for the ground control points were imported into PhotoScan prior to photo alignment. Figure 4.13 shows the generated 3D point cloud using the assisted georeferencing method. 


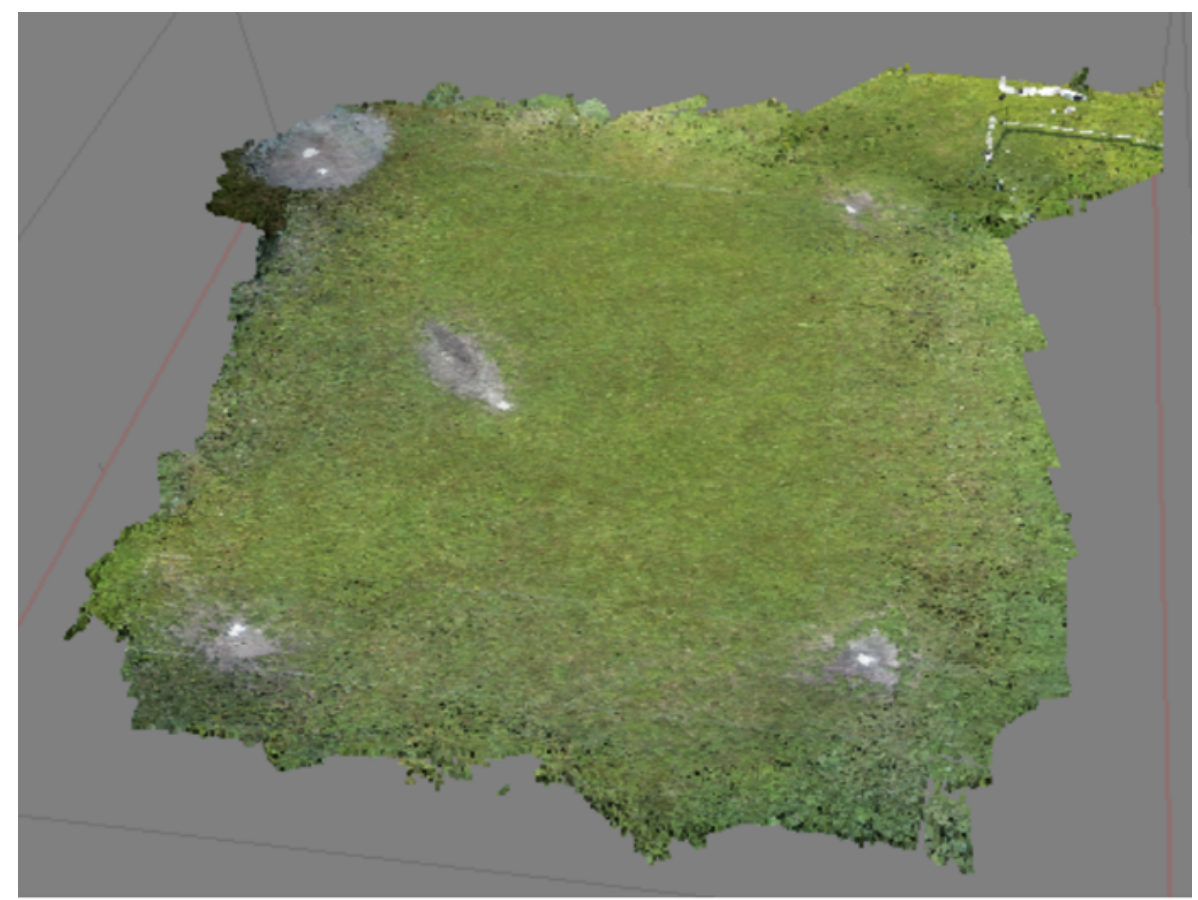

Figure 4.13: 3D point cloud generated using the assisted method of georeferencing.

Similar to the verification and validation process used in for direct georeferencing, the exterior orientation parameters determined from the indirect georeferenced 3D point cloud were exported and compared to the values obtained from the assisted georeferenced 3D point cloud. Table 4.4 summarizes the average difference between the predicted (i.e. indirect method) and calculated (i.e. assisted method) results organized by flight. 
Table 4.4: Absolute average difference between indirect and assisted results

\begin{tabular}{|c|c|c|c|}
\hline Fight & Easting $[\mathbf{m}]$ & Northing $[\mathbf{m}]$ & Altitude [m] \\
\hline $\mathbf{1}$ & 0.160 & 0.323 & 1.90 \\
\hline $\mathbf{2}$ & 0.350 & 0.358 & 1.71 \\
\hline $\mathbf{3}$ & 0.390 & 0.415 & 1.47 \\
\hline Average $[\mathrm{m}]$ & $\mathbf{0 . 3 0 0}$ & $\mathbf{0 . 3 6 5}$ & $\mathbf{1 . 6 9}$ \\
\hline
\end{tabular}

\section{Result Analysis}

As previously discussed, the first method that was used for the verification and validation of the results was to compare the values obtained from both the direct and assisted methods of georeferencing with those determined through the indirect method, calculated by PhotoScan. For both cases, the calculated coordinates of the camera's perspective center were compared with the predicted coordinates obtained using PhotoScan and the difference was calculated. The absolute average of the differences were then determined and categorized according to their respective flight number. These results were summarized in Tables 4.3 and 4.4. Furthermore, the total average values for the coordinates of the camera station were determined to be $0.382,0.610$ and $1.726 \mathrm{~m}$ as well as $0.300 \mathrm{~m}, 0.365 \mathrm{~m}$ and $0.169 \mathrm{~m}$ for the direct and assisted methods, respectively.

Prior to discussing the positional accuracy that was obtained for both methods, some research into the different methods to asses the accuracy and precision of GPS devices were researched. The Iris+ employs a u-blox LEA-6H-0-002 GPS chip which has a reported $2.5 \mathrm{~m}$ accuracy in the horizontal direction [53]. The accuracy values 
provided by u-blox correspond to a circular error probability (CEP) of 50\%, which is a measure of the device's precision. The former defines a circle of a given diameter (2.5 $\mathrm{m}$ in the case of the u-blox LEA-6H-0-002), at least $50 \%$ of the measured points are expected to lie within the perimeter defined by the circle. In addition to its reported accuracy, the u-blox LEA-6H-0-002 chip has a receiver which allows for access to satellite-based augmentation systems (SBAS), specifically the European Geostationary Navigation Overlay Service (EGNOS), the Multi-functional Satellite Augmentation System (MSAS) for Japan and the Wide Area Augmentation System (WAAS) [53], which applies for North America. The documented horizontal accuracy attainable for the u-blox chip in the presence of SBAS signals is a CEP of $2 \mathrm{~m}$ [53]; however, the documentation does not specify for which system.

The positional accuracy of GPS devices are governed by numerous factors, these include [54]:

- Ephemeris error

- Ionospheric and tropospheric delays

- Receiver noise

- Satellite geometry

- Multi-path effects

- Number of available satellites

In the absence any additional corrections, GPS devices can obtain accuracies of 2-7 $\mathrm{m}$ in ideal conditions $[54,55]$. However, these values can be improved by the WAAS through obtaining real-time correction data and accuracies of 1-2 $\mathrm{m}$ can be achieved in ideal conditions, which among others includes a clear sky $[54,55]$. Taking this 
information into consideration, the information provided by u-blox for the horizontal accuracy of the LEA-6H-0-002 chip could range from 1-2 $\mathrm{m}$ in ideal conditions if local area augmentation can be achieved. For the tests conducted on River Field, the values that were calculated in PhotoScan were treated as a known reference point and compared to the values calculated through direct georeferencing and the relative difference was determined. The values obtained in the horizontal direction indicated an accuracy of less than $\pm 1 \mathrm{~m}$, which was unexpected. However, based on the research conducted into the GPS accuracy of the u-blox employed on the Iris+, it was determined to be possible for the values to lie within a circle of 1-2 $\mathrm{m}$ with a confidence of $50 \%$. On the day and time of the flight tests, there were clear skies, low winds and at the surveying altitude there would be few obstacles that could cause multi-path interferences. Therefore, the testing conditions were likely close to the ideal conditions needed for the u-blox's accuracy to be within 1-2 m. Table 4.3 shows the determined results categorized per flight and they are within a similar range. Another factor that could influence the positional data obtained from the Iris + , is the presence of its onboard filtering through use of an Extended Kalman Filter (EKF) [49]. Based on the gyroscopes' rate, accelerometer, GPS, airspeed and barometric pressure measurements the vehicle's position, velocity and angular orientation can be estimated. An EKF can be used to reject measurements which contain significant errors [49]. The former could be a contributing factor which aided to improve the results obtained from the Pixhawk, which were used in all the calculations for the direct georeferencing results.

The second method of georeferencing that was explored using the system outlined in this thesis was assisted georeferencing. The results were analyzed the same way as the values obtained through direct georeferencing. The values for the absolute averaged difference between the reference values (i.e. indirect method) and the ones 
obtained through the combination of ground control points as well as the calculated values used for direct georeferencing were determined to all have an accuracy of less than $\pm 1 \mathrm{~m}$. There are two main elements that can have an impact on the results of the assisted method; the accuracy of the ground control points and calculated values for the camera's perspective image (determined from the direct method). The assessment of the direct georeferenced results was previously discussed and as stated in Chapter 3, the ground control points' accuracy is governed by the accuracy of the Emlid Reach RTK positioning device. Since the assisted method of georeferencing employs features of both indirect and direct methods, it was expected that the results obtained should be more precise than the values obtained solely through the direct method. The former can be seen by comparing the averaged absolute values for all combined flights summarized in Tables 4.3 and 4.4 .

During the generation of the 3D point clouds, the processing times were monitored for comparison. There are two main steps to generating a point cloud in PhotoScan, photo alignment and building a dense 3D point cloud. The times for each step are summarized in Table 4.5.

Table 4.5: Georeferencing processing times

\begin{tabular}{|c|c|c|}
\hline Method & $\begin{array}{c}\text { Photo Alignment } \\
{[\mathrm{mins}]}\end{array}$ & $\begin{array}{c}\text { Building Dense Point Cloud } \\
{[\mathrm{mins}]}\end{array}$ \\
\hline Indirect & 131 & 149 \\
\hline Direct & 130 & 158 \\
\hline Assisted & 130 & 163 \\
\hline
\end{tabular}

The photo alignment process took approximately the same amount of time for each 
method and were similar when building the dense point cloud. All times were obtained from PhotoScan's console logs. However, the values shown in Table 4.5 do not include the human interaction time, specifically for the indirect and assisted methods. The former employ the use of ground control points which must be manually placed on the images. For the survey conducted on River Field, five ground control points were used and 715 images were captured, this added approximately 30-40 minutes. Taking this into consideration, the direct georeferencing approach to surveying can indeed affect and improve the post-processing time of the images as the method can alleviate the need for as many ground control points.

The second approach that was employed to verify and validate the proposed system in this thesis was the 3D point cloud editing and processing software, CloudCompare version 2.8.1 [56]. This software's features can be used among others things, to directly compare two different point clouds and was designed to deal with very large point cloud files. The comparison requires two point clouds, one which acts as a reference and a second to compare against. For the analysis, the 3D point cloud generated using the indirect method of georeferencing was used as the reference and both the point clouds generated using direct and assisted georeferencing were compared using the cloud to cloud distance calculator. The former employs the nearest neighbor algorithm to determine for each point of the compared point cloud, the closet point from the reference point cloud and then proceeds to calculate the Euclidean distance between the two [56]. These distances can be calculated in the $x, y$ and $z$ axes.

Initially, the generated 3D point clouds obtained from the indirect and direct method of georeferencing, shown in Figure 4.14, were compared and their distances were calculated. 


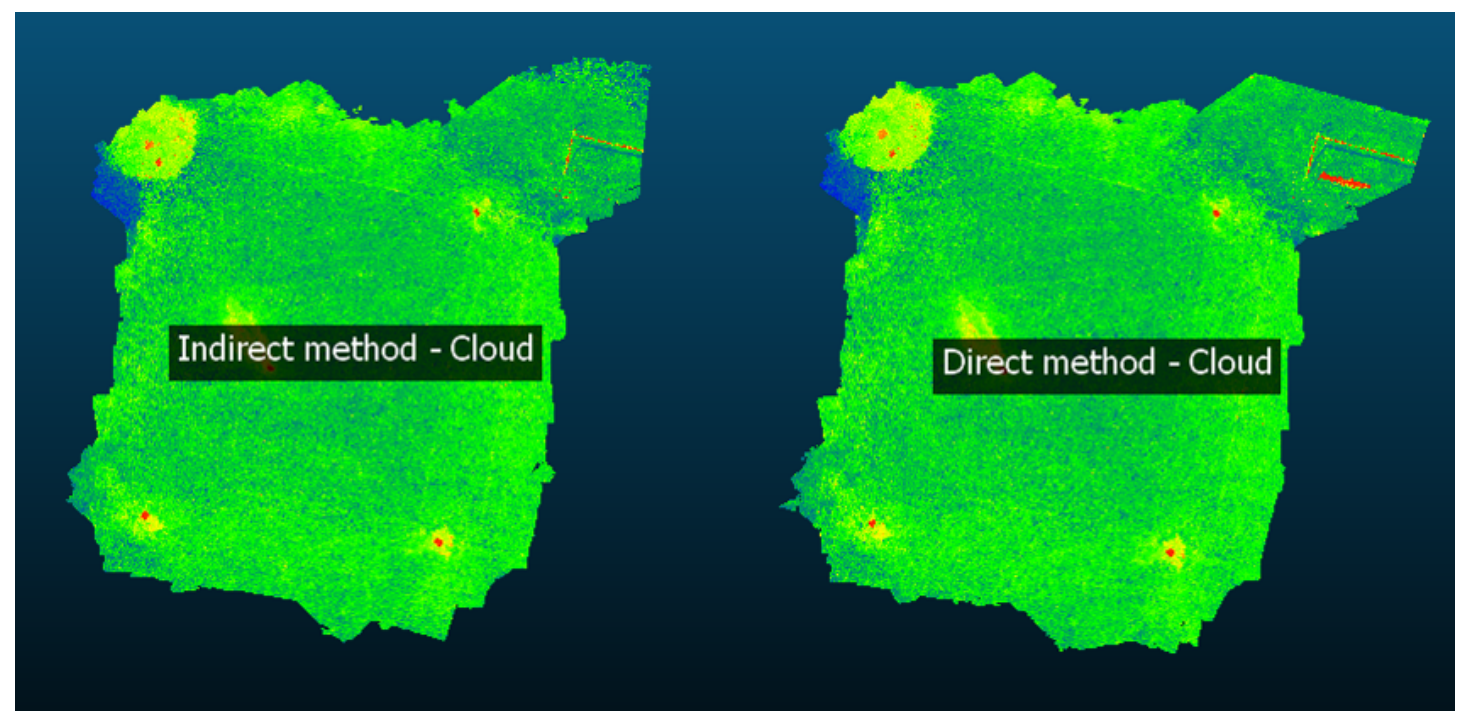

Figure 4.14: Indirect and direct 3D point clouds shown in CloudCompare.

The cloud to cloud distances were then computed and the results are shown in Figure 4.15. 


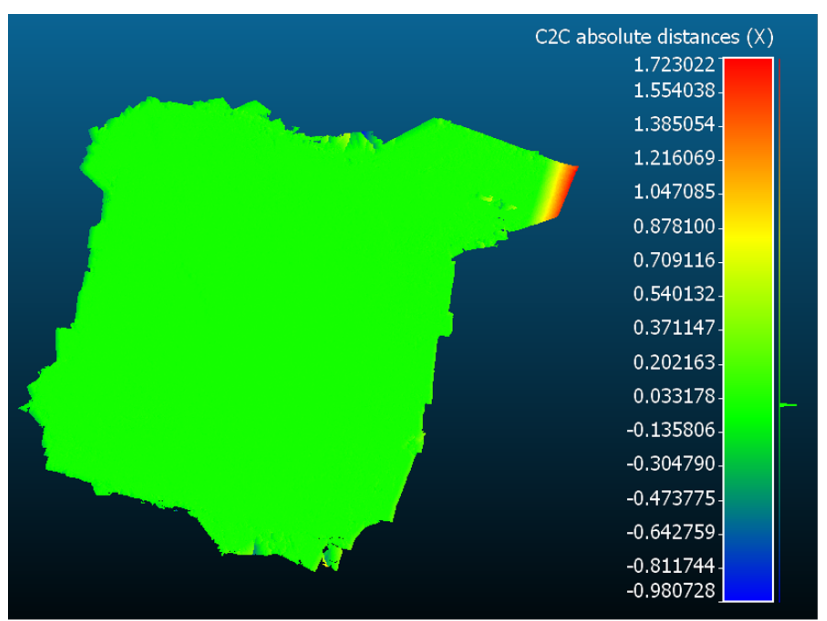

(a)

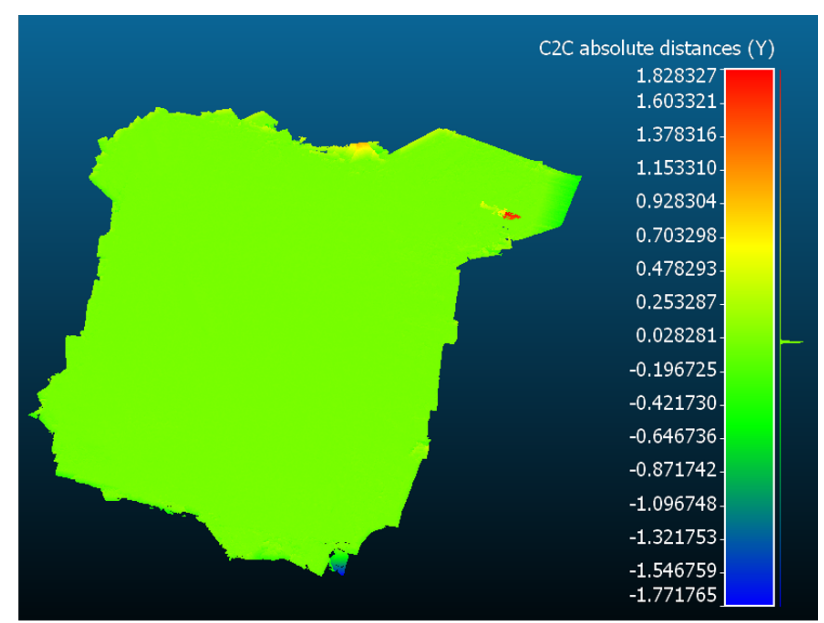

(b)

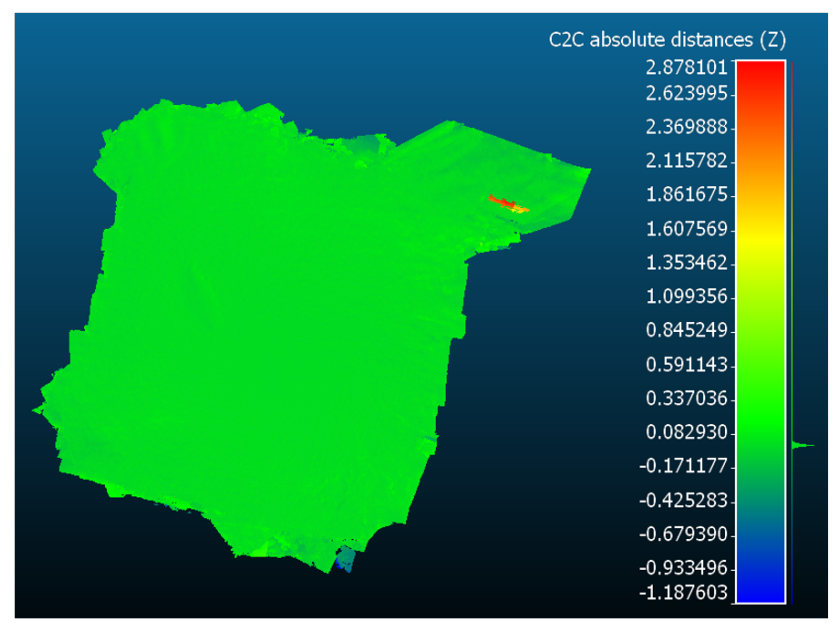

(c)

Figure 4.15: Indirect and direct 3D cloud-cloud distance comparison

Based on the images shown in Figure 4.15, it can be seen that the 3D point cloud which was generated using the direct method of georeferencing is virtually identical to the reference point cloud. 


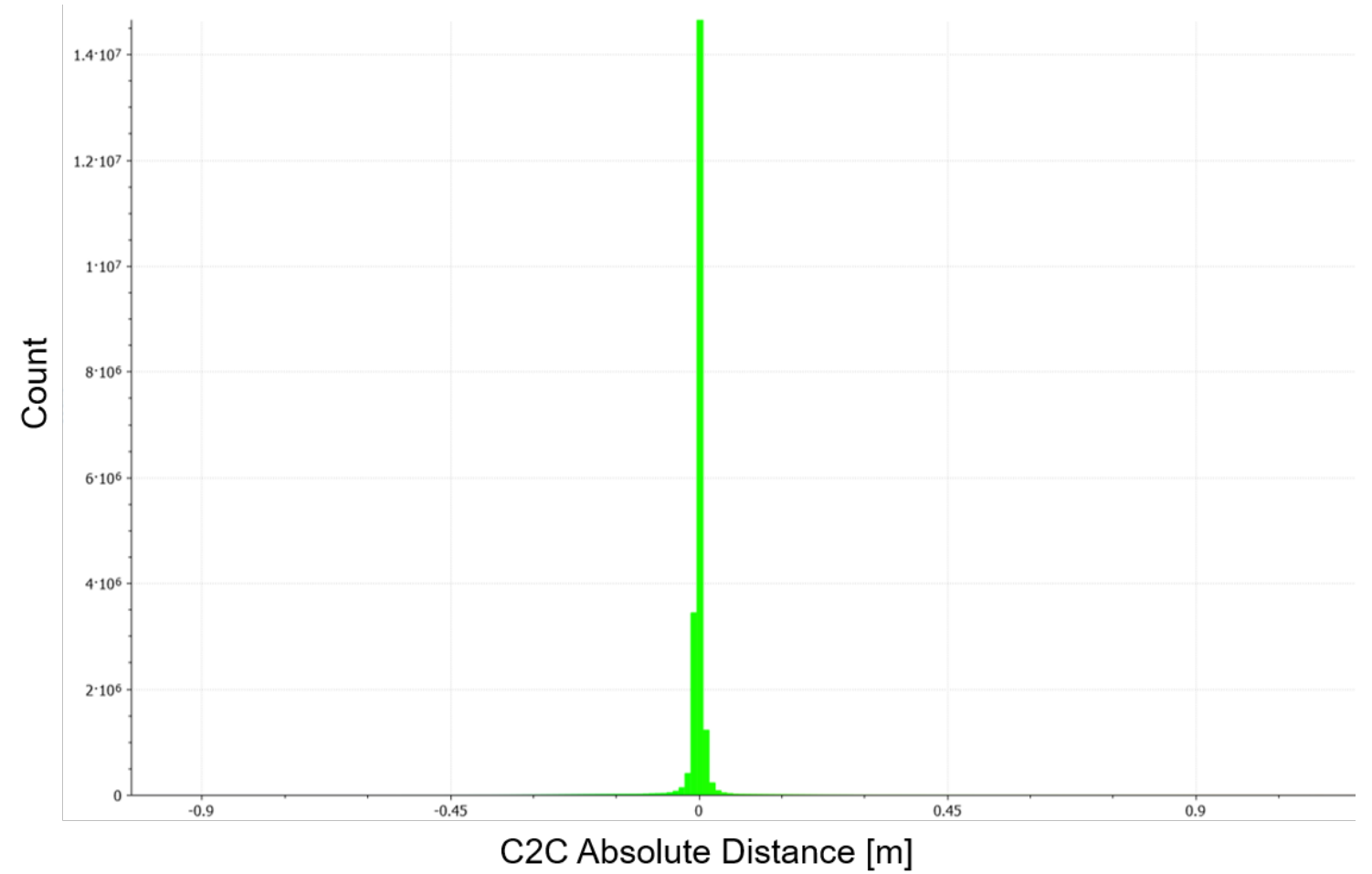

Figure 4.16: Distribution of the cloud to cloud absolute distance between the indirect and direct point clouds for the $\mathrm{x}$ axis

Figure 4.16 shows the distribution of the cloud to cloud absolute distance between the indirect and direct point clouds in the $x$ direction (Figure 4.15 a). The values primarily varied from -0.125 to $0.075 \mathrm{~m}$ with $69 \%$ of the points located in between -0.0077 and $0.0031 \mathrm{~m}$. The distributions in the $y$ and $z$ directions are shown in Section A.1 of the Appendix. The distance in the y direction (Figure $4.15 \mathrm{~b}$ ) varied between -0.140 to $0.127 \mathrm{~m}$ with $74 \%$ of the points located in between -0.0057 and $0.0055 \mathrm{~m}$. Lastly, in the $z$ direction, the values for the cloud to cloud distance were in the range of -0.150 to $0.146 \mathrm{~m}$, with $49 \%$ of the values located between -0.0063 and $0.0101 \mathrm{~m}$. This analysis process was repeated for the point cloud generated using the assisted method of georeferencing. The results for the cloud to cloud distance comparison are shown in Figure 4.17. 


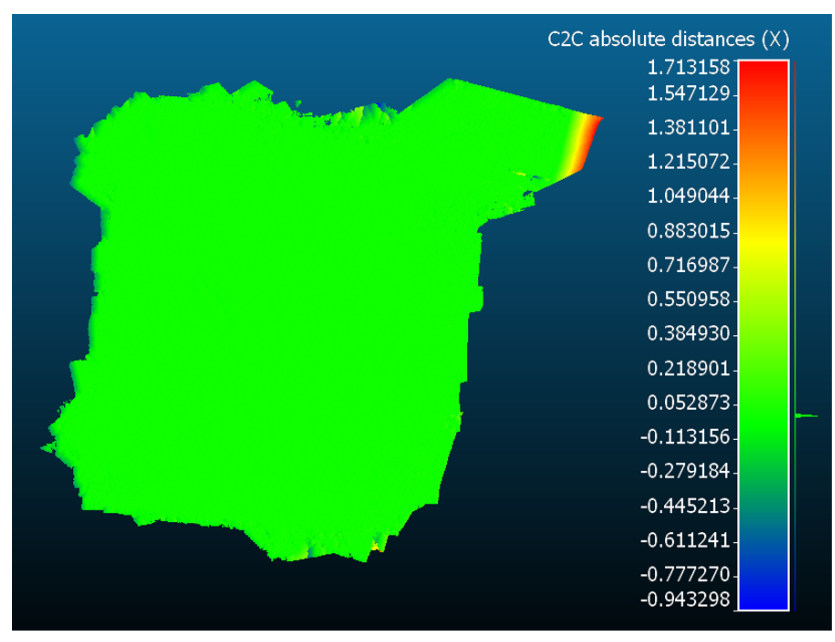

(a)

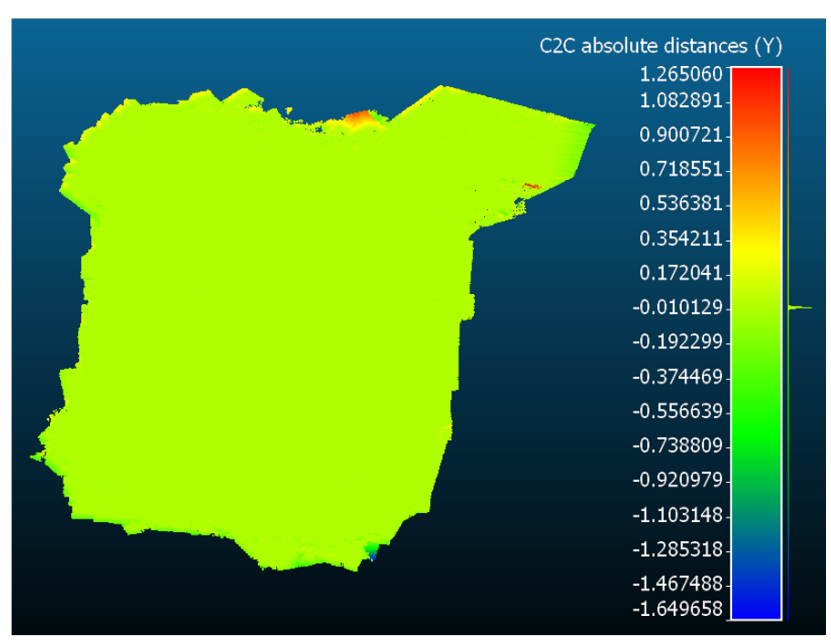

(b)

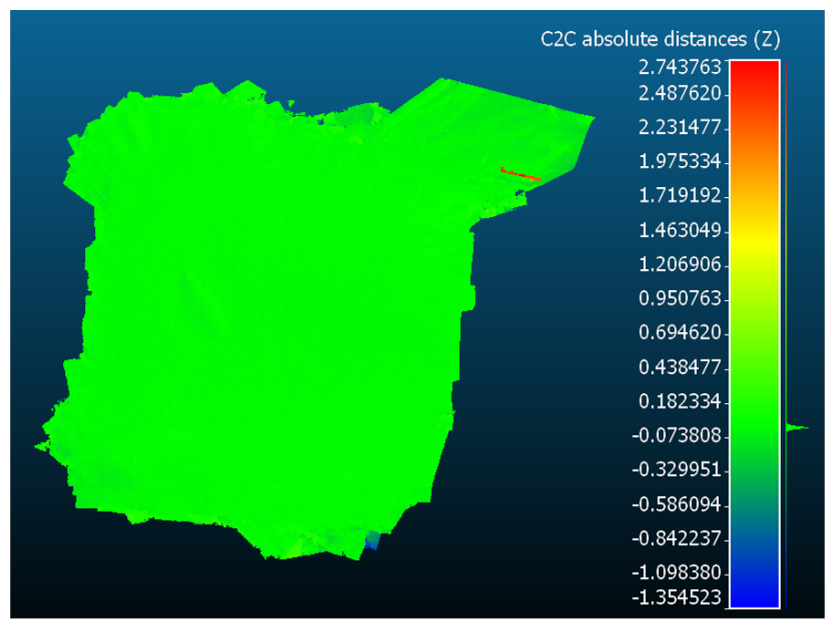

(c)

Figure 4.17: Indirect and assisted 3D cloud-cloud distance comparison

The distribution of the cloud to cloud absolute distance between the indirect and assisted point clouds are shown in Section A.2 of the Appendix. Similar to the results obtained for the direct georeferencing method, the assisted method produced values which show good agreement between both point clouds. The cloud to cloud distance in the $x$ direction varied from -0.133 to $0.073 \mathrm{~m}$, with $71 \%$ of the points located in the range of -0.0040 and $0.0064 \mathrm{~m}$. In the $y$ direction, the values were in the range of -0.100 to $0.092 \mathrm{~m}$ having $59 \%$ of the points located between -0.0016 
and $0.0100 \mathrm{~m}$. Lastly, in the $z$ direction, the distances varied from 0.153 to 0.134 $\mathrm{m}$, with $46 \%$ of the values located in the range of -0.0027 to $0.0133 \mathrm{~m}$. Overall, the CloudCompare analysis showed a similar trend as to what was observed in the first method of verification and validation. This observation is that although both the direct and assisted methods of georeferencing produce products of similar quality and accuracy, the assisted method proves to be the closest to the reference, which is the data and point cloud generated using the indirect method of georeferencing. A possible explanation for this observation could be due to the fact that the assisted method incorporates a portion of the data, specifically the positions of the ground control points, in its calculations, which are the exact same as what is used to generate the reference point cloud. 


\section{Chapter 5}

\section{Conclusions and Future Work}

\subsection{Conclusions}

An alternative method to conduct airborne photogrammetry was presented in this thesis using onboard computing and low-cost sensors. The approach explored added additional computational functionality to small uninhabited aerial vehicles to allow a custom data acquisition script, developed using Dronekit-Python, to directly associate relevant telemetry data to images being captured in real time. A survey of River Field, located on the Carleton University campus, was successfully conducted to test the feasibility and obtainable accuracy of the proposed system outlined in this thesis. The captured images were used to generate 3D point clouds using the three different methods of georeferencing, with the point cloud generated through indirect georeferencing acting as a reference for comparison. The subsequent analysis to evaluate the predicted and calculated parameters of exterior orientations showed that both the direct and assisted methods of georeferencing obtained an average accuracy of less than $\pm 1 \mathrm{~m}$ relative to the reference point cloud. Furthermore, 3D point cloud editing and processing software was employed to analyze the direct and assisted methods' 
generated point clouds relative to the point cloud obtained through indirect georeferencing. This process showed that both the tested georeferencing methods produced products of similar quality and accuracy to those obtained by the traditional method of photogrammetry. In addition to the point cloud comparisons, the position and orientation values of the UAV, which were recorded using a custom data acquisition script were compared to the telemetry logs of the UAV's autopilot to gage the efficacy of the implemented script. These comparisons indicated good agreement for the positional values recorded by the custom data acquisition script with those obtained from the UAV's autopilot. However, the former was not the case for the recordings of orientation, which showed a disagreement of $40 \%$ for the selected sample size.

As the use of UAVs for commercial applications continues to grow so will the development of new UAV technologies, which will affect the methods used to digitally capture aerial images. Specifically, the former will benefit research relating to the field of direct georeferencing, as it has the possibility of decreasing the overall cost of aerial surveying and mapping by reducing the total number of required ground control points as well as alleviating some of the post-processing of the results. Based on a review of the common methods of georeferencing, gaps in the current process were identified and an initial solution was proposed using a small uninhabited aerial vehicle. The methodology and system described in this thesis could further decrease the cost and post-processing involved in the direct georeferencing process. Presently, the synchronization between image capture times and the GPS logs represents one of the most important factors in DG, requiring a high degree of precision. The system implemented in this thesis could alleviate the need for the synchronization process as the new method relates the relevant position and orientation data of the UAV directly to the image being captured in real time. 


\section{$5.2 \quad$ Future Work}

This section discusses potential improvements and recommendations which could be implemented on the proposed system. Some suggestions for future work are:

- 3D Robotics has made the CAD models of the various components for the Iris+ available to the public, thus it would be recommended to modify the design of the UAV's body to better incorporate both the Raspberry Pi as well as the camera in order to limit errors associated with sensor placement, which can have a direct impact on the direct georeferencing results.

- Cost permitting, it could be beneficial to explore incorporating a higher precision GPS unit onto the UAV to further improve the accuracy of the measured positional values. Furthermore, incorporating a LIDAR sensor which could be mounted to the bottom of the UAV would allow for more accurate results to be obtained for the altitude.

- It is recommended to investigate alternative onboard computers for the aircraft as there were connectivity issues that arose when establishing communication between the Raspberry Pi and Pixhawk autopilot. For example, the NAVIO autopilot board from Emlid, which is an autopilot extension board for the Raspberry Pi could be researched. Alternatively, a UAV such as the 3DR Solo could be evaluated as a replacement for the Iris + as it has a built-in Linux system.

- The airborne survey conducted in this thesis was on relatively level terrain and was conducted on a day with low winds and clear skies. It would be beneficial to test the proposed system in less favorable conditions to gage the effects on accuracy. Furthermore, the survey was conducted at low altitude, thus it would be recommended to repeat the survey at varying altitudes to observe its effect 
on the precision of the results. Lastly, an additional survey could be conducted on a building or at a heritage site to evaluate the performance of the system and to compare the surveying and processing times to the traditional methods currently employed. 


\section{References}

[1] Remondino, F. and Campana, S. 3D Recording and Modelling in Archaeology and Cultural Heritage: Theory and best practices. Archaeopress Oxford, UK, 2014.

[2] Jenkins, D. and Vasigh, B. The economic impact of unmanned aircraft systems integration in the United States. Association for Unmanned Vehicle Systems International (AUVSI), 2013.

[3] Brutto, M.L., Garraffa, A., and Meli, P. Uav platforms for cultural heritage survey: first results. ISPRS Annals of the Photogrammetry, Remote Sensing and Spatial Information Sciences, 2(5):227, 2014.

[4] Ip, A. Analysis of integrated sensor orientation for aerial mapping. University of CalGary Geomatics Engineering Reports, 2005.

[5] Turner, D., Lucieer, A., and Wallace, L. Direct georeferencing of ultrahighresolution uav imagery. IEEE Transactions on Geoscience and Remote Sensing, 52(5):2738-2745, 2014.

[6] Westoby, M.J., Brasington, J., Glasser, N.F., Hambrey, M.J., and Reynolds, J.M. Structure-from-motion photogrammetry: A low-cost, effective tool for geoscience applications. Geomorphology, 179:300-314, 2012.

[7] Smith, M.W., Carrivick, J.L., and Quincey, D.J. Structure from motion photogrammetry in physical geography. Progress in Physical Geography, 40(2):247$275,2016$.

[8] Pfeifer, N., Glira, P., and Briese, C. Direct georeferencing with on board navigation components of light weight uav platforms. International Archives of the Photogrammetry, Remote Sensing and Spatial Information Sciences, 2012. 
[9] Craig, J.J. Introduction to robotics: mechanics and control, volume 3. Pearson Prentice Hall Upper Saddle River, 2005.

[10] Nex, F. and Remondino, F. Uav for 3d mapping applications: a review. Applied Geomatics, 6(1):1-15, 2014.

[11] Watts, A.C., Ambrosia, V.G., and Hinkley, E.A. Unmanned aircraft systems in remote sensing and scientific research: Classification and considerations of use. Remote Sensing, 4(6):1671-1692, 2012.

[12] Blyenburgh Co. UVS International. Internet: https://uvs-international.org/, 2016, [Aug. 19, 2017].

[13] Rinaudo, F., Chiabrando, F., Lingua, A.M., and Spanò, A.T. Archaeological site monitoring: Uav photogrammetry can be an answer. International Archives of the Photogrammetry, Remote Sensing and Spatial Information Sciences, 39(B5):583-588, 2012.

[14] Everaerts, J. The use of unmanned aerial vehicles (uavs) for remote sensing and mapping. The International Archives of the Photogrammetry, Remote Sensing and Spatial Information Sciences, 37(2008):1187-1192, 2008.

[15] Cryderman, C., Mah, S.B., and Shufletoski, A. Evaluation of uav photogrammetric accuracy for mapping and earthworks computations. Geomatica, 68(4):309$317,2014$.

[16] Kohoutek, T.K. and Eisenbeiss, H. Processing of uav based range imaging data to generate detailed elevation models of complex natural structures. International Archives of the Photogrammetry, Remote Sensing and Spatial Information Sciences, 39:405-410, 2012.

[17] He, J., Li, Y., and Zhang, K. Research of uav flight planning parameters. Positioning, 3(04):43, 2012.

[18] Harwin, S. and Lucieer, A. Assessing the accuracy of georeferenced point clouds produced via multi-view stereopsis from unmanned aerial vehicle (uav) imagery. Remote Sensing, 4(6):1573-1599, 2012.

[19] Zhang, Y. Photogrammetric processing of low altitude image sequences by unmanned airship. The International Archives of the Photogrammetry, Remote Sensing and Spatial Information Sciences, 37:751-757, 2008. 
[20] Zhang, Y., Xiong, J., and Hao, L. Photogrammetric processing of low-altitude images acquired by unpiloted aerial vehicles. The Photogrammetric Record, 26(134):190-211, 2011.

[21] Kraus, K. Photogrammetry: geometry from images and laser scans. Walter de Gruyter, 2007.

[22] Neumann, K.J. Trends for digital aerial mapping cameras. Int. Arch. Photogram. Rem. Sens. Spatial Info. Sci.(ISPRS), 28:551-554, 2008.

[23] Leachtenauer, J.C. and Driggers, R.G. Surveillance and reconnaissance imaging systems: modeling and performance prediction. Artech House, 2001.

[24] Cramer, M., Stallmann, D., and Haala, N. Direct georeferencing using gps/inertial exterior orientations for photogrammetric applications. International Archives of Photogrammetry and Remote Sensing, 33(B3/1; PART 3):198-205, 2000.

[25] Grejner-Brzezinska, D.A. Direct exterior orientation of airborne imagery with gps/ins system: Performance analysis. Navigation, 46(4):261-270, 1999.

[26] Yilmaz, H.M., Yakar, M., Gulec, S.A., and Dulgerler, O.N. Importance of digital close-range photogrammetry in documentation of cultural heritage. Journal of Cultural Heritage, 8(4):428-433, 2007.

[27] Yuan, X., Fu, J., Sun, H., and Toth, C. The application of gps precise point positioning technology in aerial triangulation. ISPRS Journal of Photogrammetry and Remote Sensing, 64(6):541-550, 2009.

[28] Linder, W. Digital photogrammetry. Springer, 2009.

[29] Bláha, M., Eisenbeiss, H., Grimm, D., and Limpach, P. Direct georeferencing of uavs. International Archives of the Photogrammetry, Remote Sensing and Spatial Information Sciences, 38(1/C22):131-136, 2011.

[30] Chiang, K., Tsai, M., and Chu, C. The development of an uav borne direct georeferenced photogrammetric platform for ground control point free applications. Sensors, 12(7):9161-9180, 2012.

[31] Rehak, M., Mabillard, R., and Skaloud, J. A micro-uav with the capability of direct georeferencing. Int. Arch. Photogramm. Remote Sens. Spatial Inf. Sci, pages 317-323, 2013. 
[32] Mostafa, M. and Hutton, J. Direct positioning and orientation systems: How do they work? what is the attainable accuracy. In Proceedings, The American Society of photogrammetry and remote sensing annual meeting, St. Louis, MO, USA, April, pages 23-27, 2001.

[33] Grejner-Brzezinska, D.A. Direct sensor orientation in airborne and land-based mapping applications. Technical report, Ohio State University. Division of Geodetic Science, 2001.

[34] Kremer, J. and Kruck, E. Integrated sensor orientation-two examples to show the potential of simultaneous gps/imu and image data processing. Theory, Technology and Realities of Inertial/GPS Sensor Orientation, 2003.

[35] Mostafa, M. Boresight calibration of integrated inertial/camera systems. In Proc. International Symposium on Kinematic Systems in Geodesy, Geomatics and Navigation-KIS, pages 440-445, 2001.

[36] Skaloud, J. Problems in direct-georeferencing by ins/dgps in the airborne environment. In ISPRS, Workshop on" Direct versus indirect methods of sensor orientation", Commission III, WG III/1, Barcelona, Spain, number TOPO-CONF1999-015, 1999.

[37] Jacobsen, K. Direct integrated sensor orientation-pros and cons. In In: Proceedings of the XXth ISPRS Congress. Citeseer, 2004.

[38] Ip, A., El-Sheimy, N., and Mostafa, M. Performance analysis of integrated sensor orientation. Photogrammetric Engineering 65 Remote Sensing, 73(1):89-97, 2007.

[39] Heipke, C., Jacobsen, K., and Wegmann, H. Analysis of the results of the oeepe test integrated sensor orientation. In OEEPE Integrated Sensor Orientation Test Report and Workshop Proceedings, Editors. Citeseer, 2002.

[40] Jacobsen, K. System calibration for direct and integrated sensor orientation. In Proceedings of the ISPRS Workshop. Working Group 1/5. Citeseer, 2003.

[41] Camera Module V2. Internet: https://www.raspberrypi.org/products/cameramodule-v2/, [Aug. 10, 2017].

[42] Raspberry Pi Model 3B. Internet: https://www.raspberrypi.org/products/raspberrypi-3-model-b/, [Aug. 10, 2017].

[43] 3D Robotics and ArduPilot. Pixhawk PX4 Autopilot. Internet: https://pixhawk.org/, [Jul. 10, 2017]. 
[44] 3D Robotics. Pixhawk Autopilot. Internet: https://3dr.com/wpcontent/uploads/2017/03/pixhawk-manual-rev7-1.pdf, [Aug. 5, 2017].

[45] Piras, M., Grasso, N., and Jabbar, A.A. Uav photogrammetric solution using a raspberry pi camera module and smart devices: Test and results. In ISPRS Archives of the Photogrammetry, Remote Sensing and Spatial Information Sciences, 2017.

[46] Ardupilot Dev Team. Mission planner documentation, 2016.

[47] 3D Robotics. Dronekit-python documentation, 2016.

[48] Agisoft. Agisoft photoscan user manual: Professional edition version 1.2, 2016.

[49] Ardupilot Dev Team. Ardupilot development site, 2016.

[50] Transport Canada. Special flight operations certificate. Internet: https://www.tc.gc.ca/eng/civilaviation/opssvs/applying-special-flightoperations-certificate.html, 2017, [Sept. 15, 2017].

[51] Emlid. Emlid Reach RTK Documentation. Internet: https://docs.emlid.com/reach/, 2017, [Sept. 10, 2017].

[52] esri. Mean Sea Level, GPS and the Geoid. Internet: http://www.esri.com/news/arcuser/0703/geoid1of3.html, 2003, [Oct. 16, 2017].

[53] u-blox. LEA-6 u-blox 6 GPS Modules Data Sheet. Internet: https://www.ublox.com/en/product/lea-6-series, 2017, [Oct. 19, 2017].

[54] Zandbergen, P.A. and Barbeau, S.J. Positional accuracy of assisted gps data from high-sensitivity gps-enabled mobile phones. The Journal of Navigation, 64(3):381-399, 2011.

[55] Andersen, H., Clarkin, T., Winterberger, K., and Strunk, J. An accuracy assessment of positions obtained using survey-and recreational-grade global positioning system receivers across a range of forest conditions within the tanana valley of interior alaska. Western Journal of Applied Forestry, 24(3):128-136, 2009.

[56] CloudCompare. Cloudcompare version 2.6.1 user manual, 2015. 


\section{Appendix A}

\section{CloudCompare Results}

\section{A.1 Indirect versus Direct}

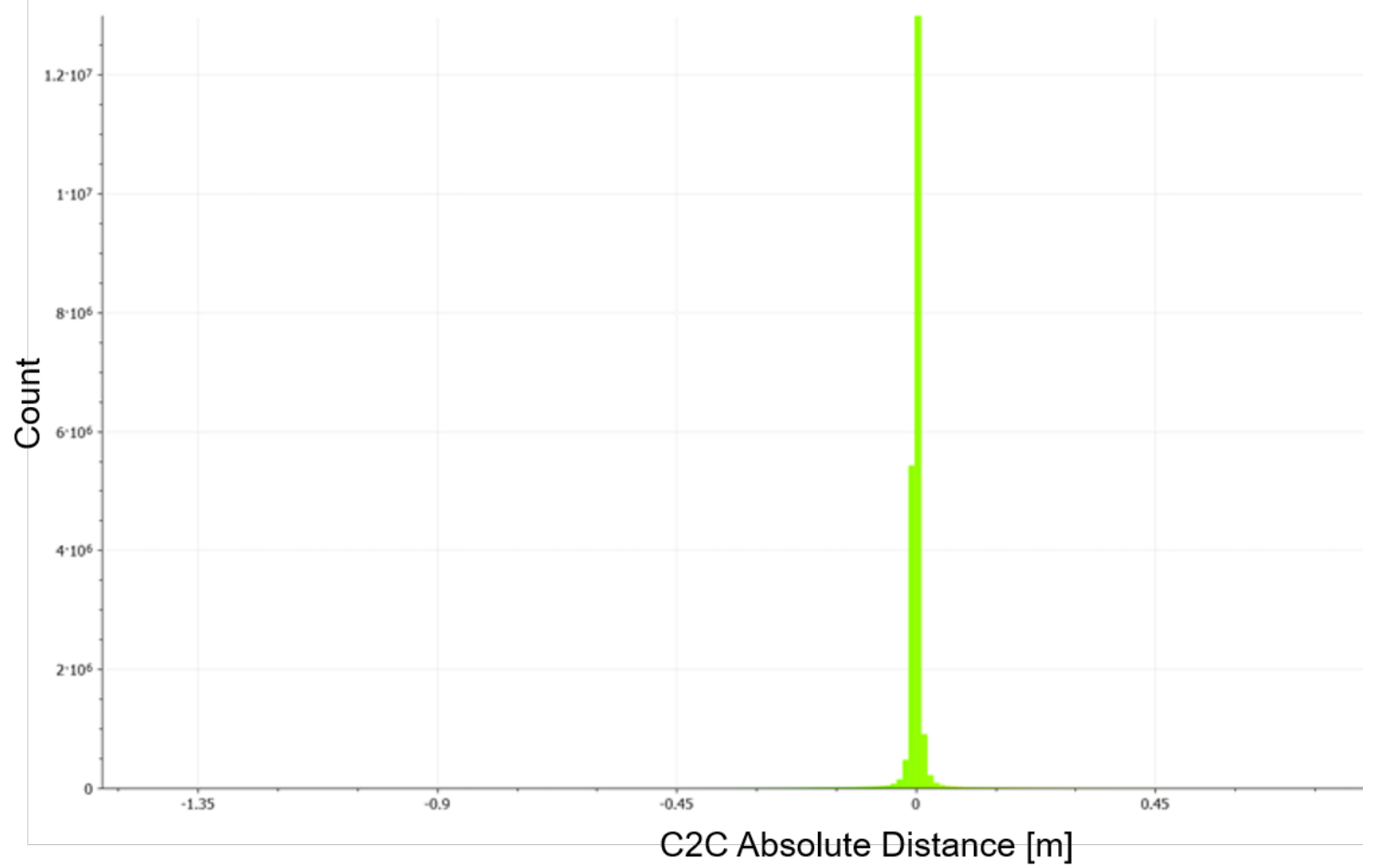

Figure A.1: Distribution of the cloud to cloud absolute distance between the indirect and direct point clouds for the $y$ axis 


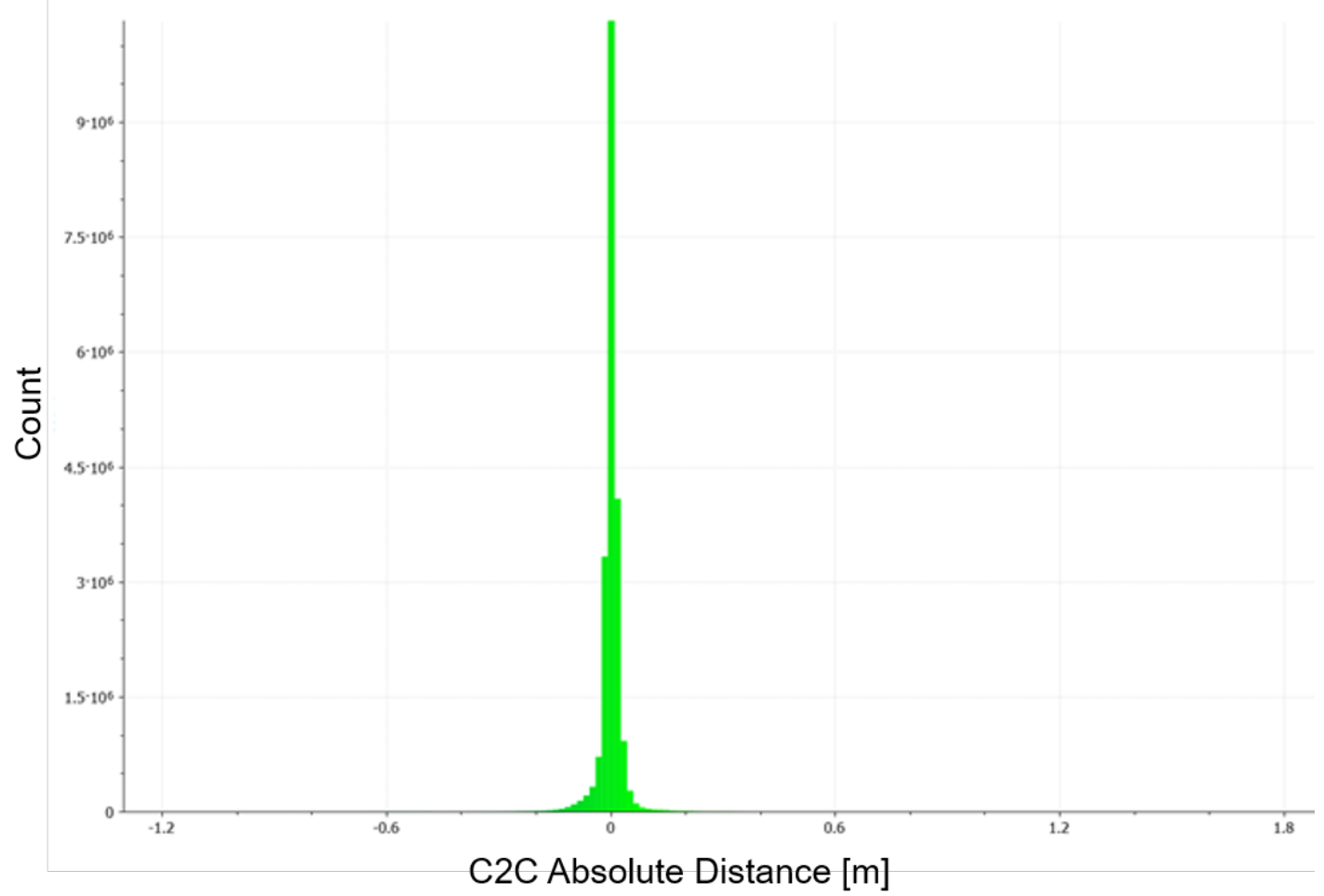

Figure A.2: Distribution of the cloud to cloud absolute distance between the indirect and direct point clouds for the $\mathrm{z}$ axis 


\section{A.2 Indirect versus Assisted}

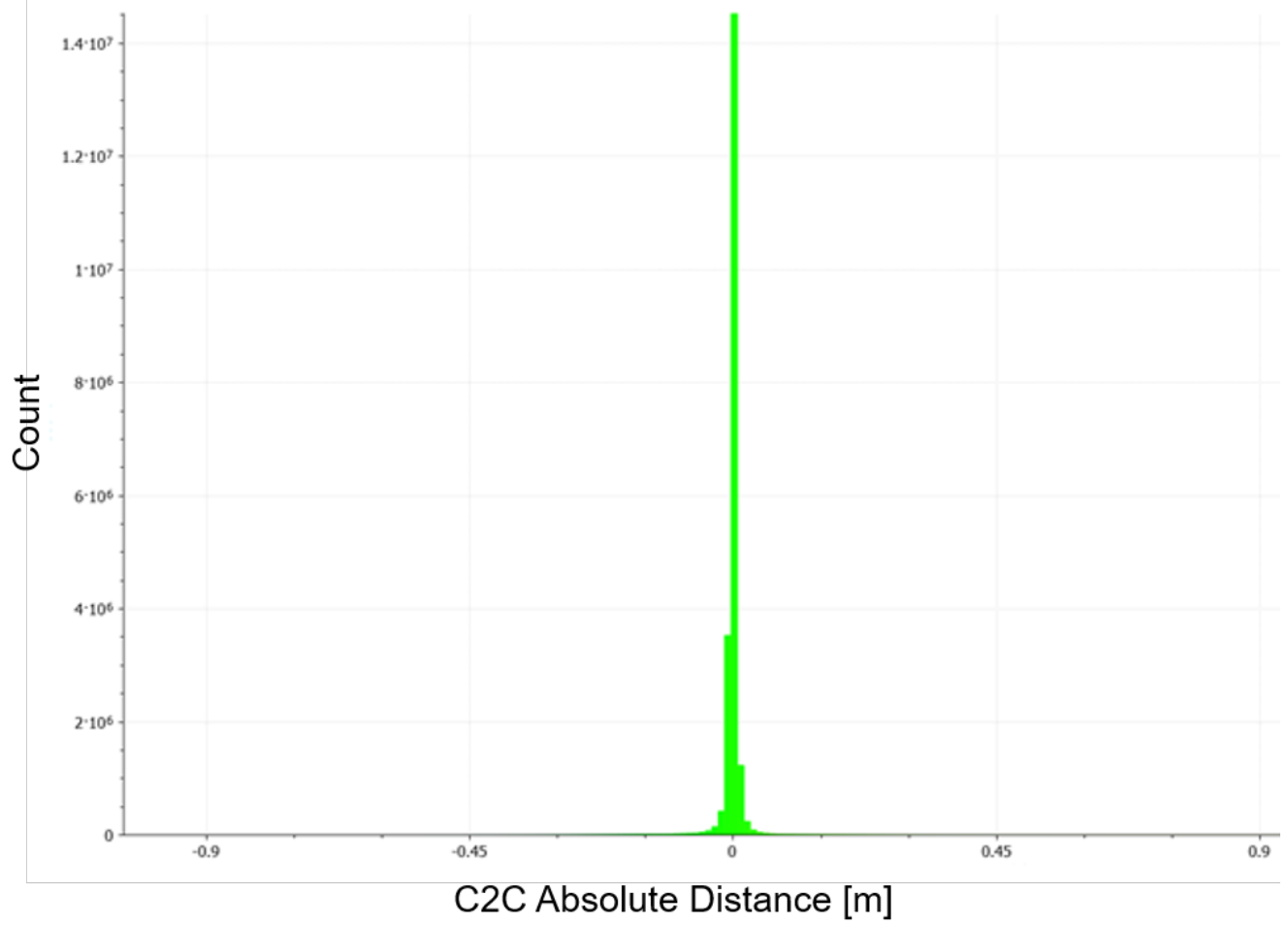

Figure A.3: Distribution of the cloud to cloud absolute distance between the indirect and assisted point clouds for the $\mathrm{x}$ axis 


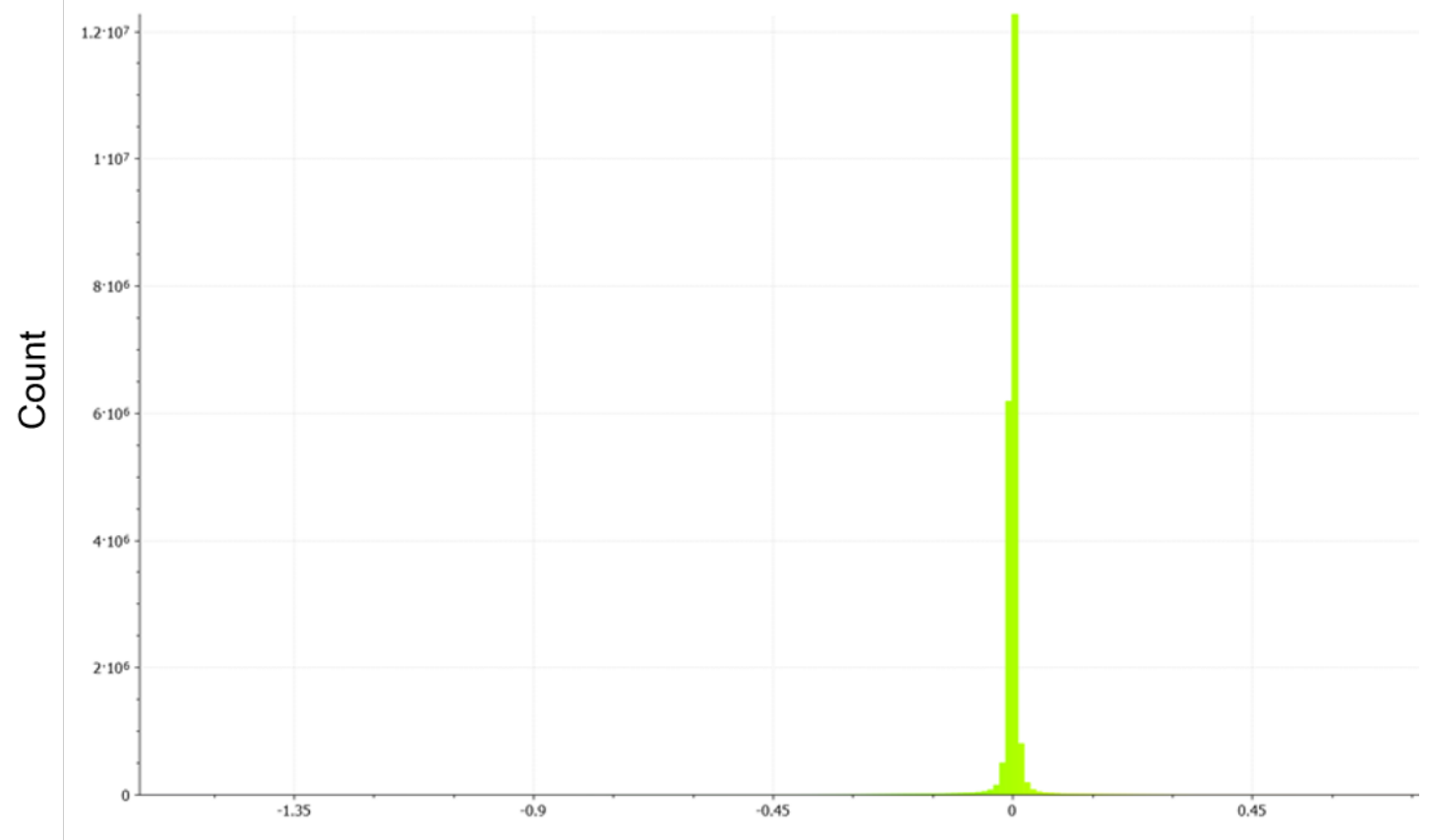

\section{C2C Absolute Distance [m]}

Figure A.4: Distribution of the cloud to cloud absolute distance between the indirect and assisted point clouds for the $\mathrm{y}$ axis 


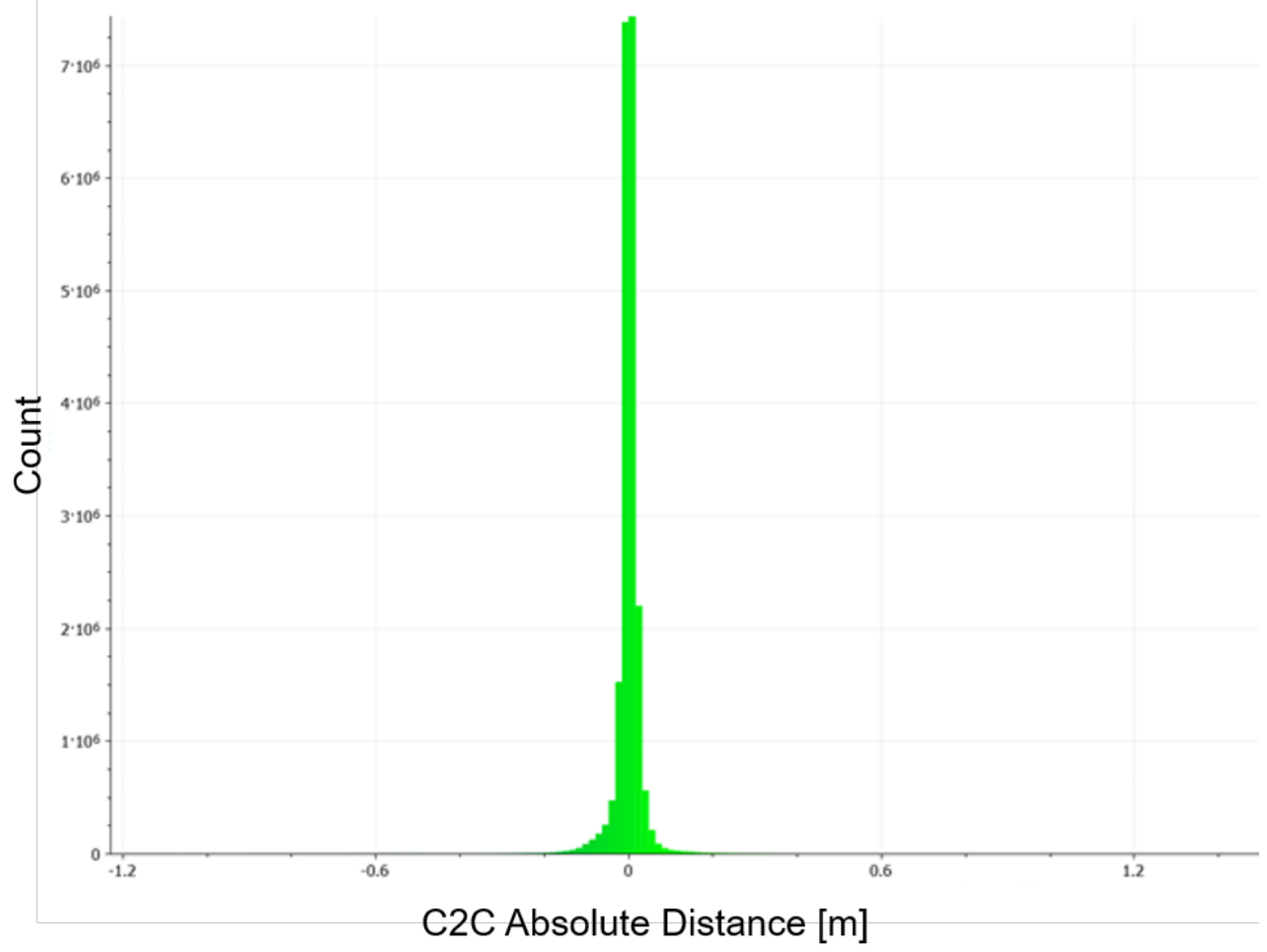

Figure A.5: Distribution of the cloud to cloud absolute distance between the indirect and assisted point clouds for the $\mathrm{z}$ axis 